\title{
GORENSTEIN RESOLUTIONS OF 3-DIMENSIONAL TERMINAL SINGULARITIES
}

\author{
TAKAYUKI HAYAKAWA
}

\begin{abstract}
Let $X$ be a 3 -dimensional terminal singularity of index $\geq 2$. We shall construct projective birational morphisms $f: Y \rightarrow X$ such that $Y$ has only Gorenstein terminal singularities and that $f$ factors the minimal resolution of a general member of $\left|-K_{X}\right|$. We also study prime divisors of $f$, especially the discrepancies of these prime divisors.
\end{abstract}

\section{§1. Introduction}

Let $X$ be a germ of a 3-dimensional terminal singularity of index $m \geq 2$ at $P$. In [Hay99] and [Hay00], we obtained a projective birational morphism $\pi: \bar{X} \rightarrow X$ such that

(i) $\bar{X}$ has only terminal singularities,

(ii) the exceptional set of $\pi$ is a prime divisor $E$, and

(iii) $K_{\bar{X}}=\pi^{*} K_{X}+\frac{1}{m} E$.

By repeating such birational morphisms, we also obtained a projective birational morphism $\tilde{\pi}: \tilde{X} \rightarrow X$ such that $\tilde{X}$ has only Gorenstein terminal singularities.

On the other hand, by [Reid87], a general member $D_{X}$ of $\left|-K_{X}\right|$ has only a rational double point at $P$. Let $D_{\bar{X}}=\pi_{*}^{-1} D_{X}$ be the birational transform. Then we see that $K_{\bar{X}}+D_{\bar{X}}=\pi^{*}\left(K_{X}+D_{X}\right)$ and that $D_{\bar{X}}$ is dominated by the minimal resolution of $D_{X}$. Thus we can expect that the restriction of $\tilde{\pi}: \tilde{X} \rightarrow X$ to the birational transform of $D_{X}$ gives the minimal resolution of $D_{X}$, and such kind of morphisms, especially various properties of exceptional divisors, should be useful in birational geometry of 3-folds.

The main purpose of this article is to construct a projective birational morphism $f: Y \rightarrow X$ such that

(i) $Y$ has only Gorenstein terminal singularities, and

Received January 30, 2003.

2000 Mathematics Subject Classification: 14B05. 
(ii) $K_{Y}+D_{Y}=f^{*}\left(K_{X}+D_{X}\right)$ and $f_{\mid D_{Y}}: D_{Y} \rightarrow D_{X}$ factors the minimal resolution of $D_{X}$, where $D_{Y}=f_{*}^{-1} D_{X}$ is the birational transform, and

(iii) the number of exceptional prime divisors of $f$ is not greater than the number of $(-2)$-curves in the minimal resolution of $D_{X}$.

We shall construct such $f: Y \rightarrow X$ explicitly in order to study prime divisors with small discrepancies over $X$. Every prime divisors with discrepancy $\leq 1$ over $P(\in X)$ appears as an exceptional divisor of $f$. Our explicit construction enables us to determine such prime divisors completely. In particular, we know that, for each $i=1, \ldots, m-1$, there is a prime divisor with discrepancy $i / m$ over $P(\in X)$. Furthermore, there is a prime divisor with discrepancy 1 over $P$ if and only if $X$ is not a cyclic quotient terminal singularity. It also follows from (iii) that the difficulty (= the number of prime divisors with discrepancies $<1$ over $P$ ) is bounded from above by the number of $(-2)$-curves in the minimal resolution of $D_{X}$.

The same kind of morphisms was already obtained by [Alex94, 5.2]. He obtained a birational morphism $g: Z \rightarrow X$ such that $Z$ has only Gorenstein terminal singularities and that $\left|-K_{Z}\right|$ is $g$-free. He developed the Minimal Model Program appropriate for linear systems and obtained $g: Z \rightarrow X$ as an application. Roughly speaking a terminalization of $K_{X}+\left|-K_{X}\right|$ over $X$ is $g: Z \rightarrow X$. Thus one has to run the Minimal Model Program over $X$ starting from some good resolution of $X$ in order to get $g$ by his method.

Our method to construct $f: Y \rightarrow X$ as above is very simple. We shall obtain $f$ starting from $X$ and make a sequence of weighted blow ups at points of indecies $>1$. By studying this process carefully, we know well about exceptional divisors of $f$. Even in the case $f$ induces the minimal resolution of $D_{X}$, our morphism $f$ is not necessarily the same as $g: Z \rightarrow X$ because of the condition (iii). In order to get $g$ from our $f: Y \rightarrow X$, we have to blow up $Y$ several times.

The contents of this article are as follows. In Section 2, we shall explain our notation and terminology. These are essentially the same as [Hay99] and [Hay00]. We shall state our main theorems and their corollaries in Section 3. After Section 4, we shall construct birational morphisms $f: Y \rightarrow X$ as above by using classification of 3-dimensional terminal singularities. Theorems in each section give a more detailed information on the exceptional divisor of the morphism $f: Y \rightarrow X$.

The author would like to thank Professor S. Mori for his invaluable suggestions and encouragement. 


\section{$\S 2 . ~ N o t a t i o n$ and preliminaries}

Throughout this section, $X$ denotes a germ of a 3 -dimensional terminal singularity at $P$ of index $m \geq 1$.

2.1. Let $\Delta$ be a (not necessarily effective) $\mathbb{Q}$-Cartier $\mathbb{Q}$-divisor on $X$ and let $\mathcal{H}_{X}$ be a linear system on $X$ which consists of $\mathbb{Q}$-Cartier Weil divisors. For a proper birational morphism $f: Y \rightarrow X$ from a normal variety $Y$, we write

$$
\begin{aligned}
K_{Y}+f_{*}^{-1} \Delta & =f^{*}\left(K_{X}+\Delta\right)+\sum a(E, X, \Delta) E, \\
\text { and } \quad K_{Y}+f_{*}^{-1} \mathcal{H}_{X} & =f^{*}\left(K_{X}+\mathcal{H}_{X}\right)+\sum a\left(E, X, \mathcal{H}_{X}\right) E
\end{aligned}
$$

where the sum runs over all prime divisors on $Y, f_{*}^{-1} \Delta$ and $f_{*}^{-1} \mathcal{H}_{X}$ are birational transforms and $a(E, X, \Delta), a\left(E, X, \mathcal{H}_{X}\right) \in \mathbb{Q}$. We call $a(E, X, \Delta)$ (resp. $\left.a\left(E, X, \mathcal{H}_{X}\right)\right)$ the discrepancy of $E$ with respect to the pair $(X, \Delta)$ (resp. $\left.\left(X, \mathcal{H}_{X}\right)\right)$. When $\Delta=0, a(E, X, \Delta)$ is denoted by $a(E, X)$. The morphism $f: Y \rightarrow X$ is called $K_{X}+\mathcal{H}_{X}$-crepant if $a\left(E, X, \mathcal{H}_{X}\right)=0$ for all $E \in \mathcal{E}(f)$.

For each prime divisor $E$ on $Y, f(E)(\subseteq X)$ is called the center of $E$ on $X$. We also say that $E$ is a prime divisor over $X$ (or $f(E))$. The discrepancy $a(E, X, \Delta)$ (resp. $\left.a\left(E, X, \mathcal{H}_{X}\right)\right)$ depends only on $\Delta\left(\right.$ resp. $\left.\mathcal{H}_{X}\right)$ and the discrete valuation on the function field of $X$ determined by $E$, and does not depend on the particular choice of $f: Y \rightarrow X$. Thus we sometimes identify prime divisors with the corresponding discrete valuations when we speak about prime divisors over $X$.

We denote the set of all exceptional prime divisors of $f$ by $\mathcal{E}(f)$, and we also define $\mathcal{E}(f, \Delta, \alpha)=\{E \in \mathcal{E}(f) \mid a(E, X, \Delta)=\alpha\}$ for $\alpha \in \mathbb{Q}$.

In [Hay99] and [Hay00], we proved the following:

TheOREM 2.2. If $P \in X$ has index $m \geq 2$, then there is a projective birational morphism $\pi: \bar{X} \rightarrow X$ such that

(i) $\bar{X}$ has only terminal singularities,

(ii) the exceptional set of $\pi$ is a prime divisor $E$, and

(iii) $K_{\bar{X}}=\pi^{*} K_{X}+\frac{1}{m} E$.

2.3. A projective birational morphism $\pi: \bar{X} \rightarrow X$ which satisfies the conditions (i), (ii), (iii) in (2.2) is called a divisorial blow up of $X$ at $P$ with discrepancy $1 / m$ (or with minimal discrepancy). We obtained such $\pi$ by 
embedding $X$ into $\mathbb{C}^{4} / \mathbb{Z}_{m}$ or $\mathbb{C}^{5} / \mathbb{Z}_{m}$ and making a weighted blow up. We remark that the exceptional divisor $E$ of $\pi$ is a divisor over $P(\in X)$, and $\pi$ is isomorphic over $X \backslash\{P\}$. In [Hay99] and [Hay00], we not only proved the existence of such divisorial blow ups, but also determined all such blow ups.

By repeating divisorial blow ups with minimal discrepancies, we also obtained the following ([Hay00]):

TheOREM 2.4. If $P \in X$ has index $m \geq 2$, then there is a sequence of projective birational morphisms

$$
X_{N} \stackrel{\pi_{N}}{\longrightarrow} X_{N-1} \longrightarrow \cdots \longrightarrow X_{1} \stackrel{\pi_{1}}{\longrightarrow} X_{0}=X
$$

such that

(i) $X_{i}$ has only terminal singularities $(i=1, \ldots, N)$, moreover $X_{N}$ has only Gorenstein terminal singularities, and

(ii) $\pi_{i}$ is a divisorial blow up at $P_{i-1} \in X_{i-1}$ with discrepancy $1 / m_{i}$, where $m_{i} \geq 2$ is the index at $P_{i-1}$.

2.5. Let $Y=X_{N}$ and let $f: Y \rightarrow X$ be the composition of $\pi_{1}, \ldots, \pi_{N}$ in (2.4). Then $f$ is a projective birational morphism which is isomorphic over $X \backslash\{P\}$ and $Y$ has only Gorenstein terminal singularities. We also know that $f$ consists of several weighted blow ups, in particular the exceptional locus of $f$ is a divisor. In this article we want to construct such $f: Y \rightarrow X$ explicitly and study exceptional prime divisors of $f$.

2.6. By [Reid87], a general member $D_{X} \in\left|-K_{X}\right|$ has a rational double point at $P$, hence there is a linear system $\mathcal{H}_{X} \subseteq\left|-K_{X}\right|$ such that a general member $D_{X} \in \mathcal{H}_{X}$ has a rational double point at $P$. We shall consider the pair $\left(X, \mathcal{H}_{X}\right)$ consisting of a germ of a 3 -dimensional terminal singularity $X$ and a linear system $\mathcal{H}_{X} \subseteq\left|-K_{X}\right|$. The Minimal Model Program which is appropriate for such pairs was developed in [Alex94] and [Cor95]. In particular, the pair $\left(X, \mathcal{H}_{X}\right)$ is canonical by [Alex94, 1.21] and we have the following:

Proposition 2.7. Let $\mathcal{H}_{X} \subseteq\left|-K_{X}\right|$ be as in (2.6) and assume that $P \in X$ has index $m \geq 2$. Let $\pi: \bar{X} \rightarrow X$ be a divisorial blow up with discrepancy $1 / m$. Then $K_{\bar{X}}+\mathcal{H}_{\bar{X}}=\pi^{*}\left(K_{X}+\mathcal{H}_{X}\right)$ and $\mathcal{H}_{\bar{X}} \subseteq\left|-K_{\bar{X}}\right|$, where $\mathcal{H}_{\bar{X}}=\pi_{*}^{-1} \mathcal{H}_{X}$. Furthermore, the birational transform $D_{\bar{X}}=\pi_{*}^{-1} D_{X}$ is normal and is dominated by the minimal resolution of $D_{X}$. In particular, $D_{\bar{X}}$ has only rational double points. 
Proof. Let $E$ be the exceptional divisor of $\pi$. Then we have $K_{\bar{X}}=$ $\pi^{*} K_{X}+\frac{1}{m} E$. Since $P$ is in the base locus of $\mathcal{H}_{X}$, we have $\pi^{*} \mathcal{H}_{X}=\mathcal{H}_{\bar{X}}+\beta E$ with $\beta \geq 1 / m$. By [Alex94, 1.21], we know that $K_{X}+\mathcal{H}_{X}$ is canonical, which implies that $\beta \leq 1 / m$. Hence we have $\beta=1 / m$, and this shows that $K_{\bar{X}}+\mathcal{H}_{\bar{X}}=\pi^{*}\left(K_{X}+\mathcal{H}_{X}\right)$.

Corollary 2.8. Assume that $P \in X$ has index $m \geq 2$. Let $f: Y \rightarrow$ $X$ be a sequence of divisorial blow ups with minimal discrepancies as in (2.4). Let $\mathcal{H}_{X} \subseteq\left|-K_{X}\right|$ be a linear system on $X$ such that a general member $D_{X}$ has only a rational double point at $P$. Then $f$ is $K_{X}+\mathcal{H}_{X}$-crepant and the induced morphism $f_{\mid D_{Y}}: D_{Y}=f_{*}^{-1} D_{X} \rightarrow D_{X}$ is dominated by the minimal resolution of $D_{X}$.

2.9. A projective birational morphism $f: Y \rightarrow X$ is called a $K_{X}+$ $\mathcal{H}_{X^{-}}$crepant Gorenstein resolution of $X$ if the following conditions are satisfied:

(i) $Y$ has only Gorenstein terminal singularities,

(ii) $f$ is isomorphic over $X \backslash\{P\}$,

(iii) the exceptional set of $f$ is a divisor, and

(iv) $K_{Y}+f_{*}^{-1} \mathcal{H}_{X}=f^{*}\left(K_{X}+\mathcal{H}_{X}\right)$, i.e., $f$ is $K_{X}+\mathcal{H}_{X}$-crepant.

The main purpose of this article is to construct a $K_{X}+\mathcal{H}_{X}$-crepant Gorenstein resolution of $X$ explicitly by using only weighted blow ups, and study its exceptional prime divisors.

The following lemma will be used to compute the discrepancies of prime divisors over $X$ :

Lemma 2.10. Let $\pi: \bar{X} \rightarrow X$ be a divisorial blow up with discrepancy $\alpha, E$ be the exceptional divisor of $\pi$, and let $\Delta$ be a $\mathbb{Q}$-Cartier divisor on $X$. Then, for every prime divisor $F$ over $\bar{X}$, we have $a(F, X, \Delta)=$ $a\left(F, \bar{X}, \pi^{*} \Delta-\alpha E\right)$.

Proof. Let $\psi: Z \rightarrow \bar{X}$ be a projective birational morphism and let $g=\pi \circ \psi: Z \rightarrow X$. Since $\pi^{*} K_{X}=K_{\bar{X}}-\alpha E$, we have $g^{*}\left(K_{X}+\Delta\right)=$ $\psi^{*}\left(K_{\bar{X}}+\pi^{*} \Delta-\alpha E\right)=K_{Z}-\sum a\left(F, \bar{X}, \pi^{*} \Delta-\alpha E\right) F$.

By a similar method, we can prove the following:

Lemma 2.11. Let $g: Z \rightarrow X$ be a projective birational morphism such that $Z$ has only Gorenstein terminal singularities and that the exceptional set of $g$ is a divisor. Then every prime divisor over $P$ with discrepancies $\leq 1$ appears as a g-exceptional divisor on $Z$. 
Proof. Let $F$ be a prime divisor over $P$ and assume that the center of $F$ on $Z$ is not a divisor. Let $h: W \rightarrow Z$ be a projective birational morphism such that $F$ is a divisor on $W$. Since $h(F)$ lies in the exceptional set of $f$, and since $Z$ has only Gorenstein terminal singularities, we have $a(F, X)>a(F, Z) \geq 1$.

As we saw in [Hay99] and [Hay00], for the divisorial blow up $\pi: \bar{X} \rightarrow$ $X$ with discrepancy $1 / m, \bar{X}$ has cyclic quotient terminal singularities in many cases. We can resolve these singularities and analyse the exceptional divisors by using the following ([Reid87, 5.7], see also [Hay99, 5.1]):

LEMMA 2.12. Let $X=(x, y, z) / \mathbb{Z}_{m}(\alpha,-\alpha, 1)$ be a germ of a 3-dimensional cyclic quotient terminal singularity, where $m \geq 2$ and $(m, \alpha)=1$. Let $\mathcal{H}_{X} \subseteq\left|-K_{X}\right|$ be a linear system such that a general member has only a rational double point. Then there is a projective birational morphism $f: Y \rightarrow X$ which satisfies the following conditions:

(i) $Y$ is non-singular and $f$ is $K_{X}+\mathcal{H}_{X}$-crepant.

(ii) $\mathcal{E}(f)=\left\{E_{1}, \ldots, E_{m-1}\right\}$ with $a\left(E_{i}, X\right)=i / m(i=1, \ldots, m-1)$. Let $\Delta_{j}=\operatorname{div}_{X}\left(f_{j}(x, y, z)\right)$ be a $\mathbb{Q}$-Cartier divisor, where $f_{j} \in \mathbb{C}\{x, y, z\}$ is a $\mathbb{Z}_{m}$-semi-invariant, and let $\Delta=\sum d_{j} \Delta_{j}$ be a $\mathbb{Q}$-Cartier $\mathbb{Q}$-divisor with $d_{j} \in \mathbb{Q}$. Then we have

(iii) $a\left(E_{i}, X, \Delta\right)=i / m-\sum d_{j} \sigma_{i}-w t\left(f_{j}(x, y, z)\right)$, where $\sigma_{i}-w t(x, y, z)=$ $(\langle\alpha i / m\rangle,\langle-\alpha i / m\rangle, i / m)$.

Furthermore if a general member $D_{X} \in \mathcal{H}_{X}$ has only a rational double point of type $A_{m-1}$, then

(iv) $f$ induces the minimal resolution $f_{\mid D_{Y}}: D_{Y}=f_{*}^{-1} D_{X} \rightarrow D_{X}$ of $D_{X}$.

2.13. The morphism $f: Y \rightarrow X$ obtained in (2.12) is called the economic resolution of $X=(x, y, z) / \mathbb{Z}_{m}(\alpha,-\alpha, 1)$. This is also obtained by a succession of divisorial blow ups with minimal discrepancies.

2.14. In this article we follow the notation of [Hay99] and [Hay00]. We shall use weighted blow ups in order to construct a $K_{X}+\mathcal{H}_{X}$-crepant Gorenstein resolution of $X$. The notion of weighted blow ups is given in [Hay99, 3.2], and we shall recall this briefly here. If $X$ is embedded in $(x, y, z, u) / \mathbb{Z}_{m}(\alpha, \beta, \gamma, \delta)$, and if $\sigma=\frac{1}{m}(a, b, c, d)$ is a weight, then we can define the $\sigma$-blow up $\pi_{\sigma}: X_{\sigma} \rightarrow X$. In this article, this is also called the blow up with weight $(x, y, z, u)=\frac{1}{m}(a, b, c, d)$. The variety $X_{\sigma}$ is covered 
by four affine open sets, which are called $x$-chart, $y$-chart, $z$-chart and $u$ chart. The structure of these affine open sets and the morphism $\pi_{\sigma}$ are described explicitly in [Hay99, 3.2]. We sometimes have to embed $X$ into $(x, y, z, u, t) / \mathbb{Z}_{m}(\alpha, \beta, \gamma, \delta, \epsilon)$. In this case, we can define the weighted blow ups by a similar method.

For $r \in \mathbb{R}$, we define the round down of $r$ by $\lfloor r\rfloor=\max \{t \in \mathbb{Z} \mid t \leq r\}$ and the round up of $r$ by $\lceil r\rceil=-\lfloor-r\rfloor$. We denote the fractional part of $r$ by $\langle r\rangle=r-\lfloor r\rfloor$.

\section{$\S 3$. Main results and their corollaries}

In this section, we shall state our main results of this article. Throughout this section, $X$ denotes a germ of a 3-dimensional terminal singularity at $P(\in X)$ and assume that $X$ has index $m \geq 2$ at $P$.

The main results of this article are summarized as follows:

THEOREM 3.1. Let $\mathcal{H}_{X} \subseteq\left|-K_{X}\right|$ be a linear system which contains a member $D_{X}$ with only a rational double point. Then there is a $K_{X}+$ $\mathcal{H}_{X}$-crepant Gorenstein resolution $f: Y \rightarrow X$ such that the number of exceptional prime divisors of $f$ is not greater than the number of (-2)curves in the minimal resolution of $D_{X}$.

We not only estimate the number of exceptional prime divisors of $f$, but also determine the discrepancies $a(E, X)$ for each $E \in \mathcal{E}(f)$.

The number of prime divisors with discrepancies $\leq 1$ (or $<1$ ) over $P$ only depends on $X$. As for these divisors, we obtain the following:

THEOREM 3.2. For each $i=1,2, \ldots, m-1$, there is a prime divisor $F_{i}$ over $P$ such that $a\left(F_{i}, X\right)=i / m$. Furthermore, there is a prime divisor $F_{m}$ over $P$ with $a\left(F_{m}, X\right)=1$ if and only if $X$ is not a cyclic quotient terminal singularity.

We remark that the first part of (3.2) is already obtained by [Sho96]. Our proof is different from the one given in [Sho96].

We shall prove (3.1) and (3.2) by using classification of 3-dimensional terminal singularities and weighted blow ups of these singularities. A more detailed description of a $K_{X}+\mathcal{H}_{X}$-crepant Gorenstein resolution $f: Y \rightarrow X$ and its exceptional prime divisors will be given in theorems in the following sections. For example, if $X$ is of type (cD/3), then (6.4), (6.6) and (6.9) 
give us an information of the exceptional prime divisors of $f$. By these theorems, we know that (3.1) and (3.2) are true if $X$ is of type $(\mathrm{cD} / 3)$.

As an easy consequence of (2.11) and (3.1), we obtain:

Corollary 3.3. Let $\mathcal{H}_{X}$ and $D_{X}$ be as in (3.1). Then the number of prime divisors over $P$ with discrepancies $\leq 1$ is not greater than the number of $(-2)$-curves in the minimal resolution of $D_{X}$. In particular, the difficulty of $X$ has the same bound from above.

A projective birational morphism $\bar{f}: \bar{Y} \rightarrow X$ is called an economic resolution of $X$ ([Reid87, 6.5]) if the following conditions are satisfied:

(i) $\bar{Y}$ has only Gorenstein terminal singularities, and

(ii) the exceptional set of $\bar{f}$ is a divisor, and $\mathcal{E}(\bar{f})$ is exactly the set of all prime divisors with discrepancies $<1$ over $P(\in X)$.

In [Reid87, 6.5], it is considered that the economic resolution is a candidate of a good partial resolution of $X$. In fact, if $X$ is cyclic quotient terminal singularity, then there is an economic resolution $\bar{f}: \bar{Y} \rightarrow X$ and $\bar{Y}$ is non-singular (cf. (2.12)). However the existence of prime divisors with discrepancies 1 over $P$ shows that there are no economic resolutions in the above sense:

PRoposition 3.4. If there is a prime divisor with discrepancy 1 over $P(\in X)$, then $X$ has no economic resolutions. In particular, if $X$ is not a cyclic quotient terminal singularity, then $X$ has no economic resolutions.

Proof. If $\bar{f}: \bar{Y} \rightarrow X$ is a economic resolution of $X$, then $\mathcal{E}(\bar{f})$ consists of prime divisors with discrepancies $<1$. On the other hand, by (2.11), prime divisors with discrepancies 1 must be contained in $\mathcal{E}(\bar{f})$. The second part follows from (3.2).

Thus we have to change conditions on singularities of $\bar{Y}$ or discrepancies of elements of $\mathcal{E}(\bar{f})$ to get such partial resolutions of $X$. These materials will be treated in [Hay05].

\section{$\S 4$. Terminal singularities of type $(\mathrm{cA} / \mathrm{m})$}

4.1. Let $X$ be a germ of a 3-dimensional terminal singularity at $P$. We assume that $X$ is of type $(\mathrm{cA} / m)$ throughout this section. Then there is an embedding $X \hookrightarrow(x, y, z, u) / \mathbb{Z}_{m}(\alpha,-\alpha, 1,0)$ such that

$$
X=\{x y+f(z, u)=0\} / \mathbb{Z}_{m}(\alpha,-\alpha, 1,0),
$$


where $f(z, u) \in\left(z^{m}, u\right) \mathbb{C}\left\{z^{m}, u\right\}$ is a $\mathbb{Z}_{m}$-invariant and $(\alpha, m)=1$.

For each positive integer $i$, we denote $\tau_{i}$-wt $(z)=i / m, \tau_{i}-w t(u)=1$, and define $w_{i}(X)=\tau_{i}$-wt $(f(z, u))$. Since $f(z, u)$ is a $\mathbb{Z}_{m}$-invariant and has no constant terms, we see that $w_{i}(X)$ are all positive integers. We also define $w_{0}(X)=0$.

Since the cyclic group $\mathbb{Z}_{m}$ acts freely on $\{x y+f(z, u)=0\} \backslash\{(0)\}$, we see that $u^{w} \in f(z, u)$ for some positive integer $w$. The smallest such integer $w$ is called the axial weight of $X$ and we shall denote it by $\operatorname{aw}(X)$ (cf. [Hay99, 2.6]).

Let $\mathcal{H}_{X} \subseteq\left|-K_{X}\right|$ be a linear system such that a general member has only a rational double point at $P$. It is easy to see that a general member of $\left|-K_{X}\right|$ has a rational double point of type $A_{\text {maw }(X)-1}$.

LEMmA 4.2. (1) For each positive integer $i$, we have $w_{i}(X) \leq w_{i+1}(X)$ and $w_{i+1}(X)-w_{i}(X) \geq w_{i+2}(X)-w_{i+1}(X)$.

(2) There is a positive integer $l=l(X)$ such that $w_{l-1}(X)<w_{l}(X)=$ aw $(X)$.

Proof. (1) The proof of the first inequality is obvious. There is a monomial $M=z^{m p} u^{q} \in f(z, u)$ such that $w_{i+1}(X)=\tau_{i+1}-w t(M)=(i+$ 1) $p+q$. Then we have $w_{i}(X) \leq \tau_{i}-w t(M)=i p+q=w_{i+1}(X)-p$. Similarly $w_{i+2}(X) \leq w_{i+1}(X)+p$. Hence we obtain the inequality $w_{i+1}(X)-w_{i}(X) \geq$ $w_{i+2}(X)-w_{i+1}(X)$.

(2) Since $u^{\text {aw }(X)} \in f(z, u)$, we have $w_{i}(X)=\operatorname{aw}(X)$ for all sufficiently large integer $i$. Thus the existence of such $l=l(X)$ follows from (1).

4.3. By (4.2), we get a sequence of strictly increasing positive integers

$$
\left(w_{1}(X), w_{2}(X), \ldots, w_{l}(X)\right)
$$

where $w_{l}(X)=\operatorname{aw}(X)$. This is called the weight sequence of $X$ as type $(\mathrm{cA} / m)$. We also call $l=l(X)$ the length of the weight sequence. We remark that $w_{l}(X)=w_{l+1}(X)=\cdots=\operatorname{aw}(X)$.

In the following, we shall consider the pair $\left(X, \Delta_{X}(k)\right)$, where $X$ is as in (4.1.1) and $\Delta_{X}(k)=-\frac{k}{m} \operatorname{div}_{X}(u)$ with $k \in \mathbb{Q}$. This allows us to work inductively. Though $\Delta_{X}(k)$ is not an effective divisor, the discrepancy $a\left(G, X, \Delta_{X}(k)\right)$ makes sense for each prime divisor $G$ over $X$.

Proposition 4.4. Let $X, w_{i}(X), l(X), \Delta_{X}(k), \mathcal{H}_{X}$ be as in (4.1) and (4.3). Let $a, b$ be positive integers satisfying $a+b=w_{1}(X), a \equiv \alpha(\bmod m)$. 
Then there is a $K_{X}+\mathcal{H}_{X}$-crepant birational morphism $f_{1}: X_{1} \rightarrow X$ such that

(i) $\mathcal{E}\left(f_{1}\right)=\left\{E, F_{1}, \ldots, F_{a-1}, F_{1}^{\prime}, \ldots, F_{b-1}^{\prime}\right\}$ with

$$
\begin{aligned}
a\left(E, X, \Delta_{X}(k)\right) & =(k+1) / m, \\
a\left(F_{i}, X, \Delta_{X}(k)\right) & =(k+\lceil m i / a\rceil) / m, \quad i=1, \ldots, a-1, \\
a\left(F_{j}^{\prime}, X, \Delta_{X}(k)\right) & =(k+\lceil m j / b\rceil) / m, \quad j=1, \ldots, b-1 .
\end{aligned}
$$

(ii) If $l(X)=1$, then $X_{1}$ has only Gorenstein terminal singularities.

(iii) If $l(X)>1$, then $X_{1}$ is Gorenstein terminal outside one point $P_{1}$, and there is an open neighborhood $P_{1} \in U \subseteq X_{1}$ such that

$$
U \simeq\left\{x y+f\left(z u^{1 / m}, u\right) / u^{w_{1}(X)}=0\right\} / \mathbb{Z}_{m}(\alpha,-\alpha, 1,0) .
$$

We have $a\left(G, X, \Delta_{X}(k)\right)=a\left(G, U, \Delta_{U}(k+1)\right)$ for each prime divisor $G$ over $P_{1}$.

Proof. We embed $X$ as in (4.1.1) and construct a blow up $g_{1}: Y_{1} \rightarrow X$ with weight $(x, y, z, u)=\frac{1}{m}(a, b, 1, m)$. By [Hay99, 6.4], we see that $g_{1}$ is divisorial with discrepancy $1 / m$ and that $Y_{1}$ is Gorenstein outside the origin $Q_{1}$ of the $x$-chart $U_{1}$, the origin $Q_{2}$ of the $y$-chart $U_{2}$ and the origin $Q_{4}$ of the $u$-chart $U_{4}$.

Let $\Delta_{Y_{1}}(k)=g_{1 *}^{-1} \Delta_{X}(k)$ and let $E_{1}$ be the exceptional divisor of $g_{1}$. Then we have $g_{1}^{*} \Delta_{X}(k)-\frac{1}{m} E_{1}=\Delta_{Y_{1}}(k)-\frac{k+1}{m} E_{1}$.

We shall resolve singularities $Q_{1}$ and $Q_{2}$ in $Y_{1}$. We have

$$
\begin{aligned}
U_{1} & =\left\{\bar{y}+f\left(\bar{x}^{1 / m} \bar{z}, \bar{x} \bar{u}\right) / \bar{x}^{w_{1}(X)}=0\right\} / \mathbb{Z}_{a}(m,-b,-1,-m) \\
& \simeq(\bar{x}, \bar{z}, \bar{u}) / \mathbb{Z}_{a}(-m, 1, m)
\end{aligned}
$$

with $\Delta_{Y_{1}}(k)_{\mid U_{1}}=-\frac{k}{m} \operatorname{div}_{U_{1}}(\bar{u})$ and $E_{1 \mid U_{1}}=\operatorname{div}_{U_{1}}(\bar{x})$. Similarly we have $U_{2} \simeq(\bar{y}, \bar{z}, \bar{u}) / \mathbb{Z}_{b}(-m, 1, m)$ with $\Delta_{Y_{1}}(k)_{\mid U_{2}}=-\frac{k}{m} \operatorname{div}_{U_{2}}(\bar{u})$ and $E_{1 \mid U_{2}}=$ $\operatorname{div}_{U_{2}}(\bar{y})$. Let $h_{1}: X_{1} \rightarrow Y_{1}$ be the economic resolution of $Q_{1}$ and $Q_{2}$ (cf. $(2.12))$. Then we have

$$
\mathcal{E}\left(h_{1}\right)=\left\{F_{1}, \ldots, F_{a-1}, F_{1}^{\prime}, \ldots, F_{b-1}^{\prime}\right\}
$$

where $F_{i}\left(\right.$ resp. $\left.F_{j}^{\prime}\right)$ is the prime divisor over $Q_{1}\left(\operatorname{resp} . Q_{2}\right)$ with $a\left(F_{i}, Y_{1}\right)=$ $i / a(i=1, \ldots, a-1)\left(\right.$ resp. $\left.a\left(F_{j}^{\prime}, Y_{1}\right)=j / b(j=1, \ldots, b-1)\right)$. By $(2.10)$ 
and (2.12), we see that

$$
\begin{aligned}
a\left(F_{i}, X, \Delta_{X}(k)\right) & =a\left(F_{i}, Y_{1}, g_{1}^{*} \Delta_{X}(k)-\frac{1}{m} E_{1}\right) \\
& =a\left(F_{i}, Y_{1}, \Delta_{Y_{1}}(k)-\frac{k+1}{m} E_{1}\right) \\
& =a\left(F_{i}, U_{1},-\frac{k}{m} \operatorname{div}_{U_{1}}(\bar{u})-\frac{k+1}{m} \operatorname{div}_{U_{1}}(\bar{x})\right) \\
& =\frac{i}{a}+\frac{k}{m}\left\langle\frac{m i}{a}\right\rangle+\frac{k+1}{m}\left\langle-\frac{m i}{a}\right\rangle \\
& =\frac{k}{m}+\frac{1}{m}\left\lceil\frac{m i}{a}\right\rceil .
\end{aligned}
$$

Similarly we have $a\left(F_{j}^{\prime}, X, \Delta_{X}(k)\right)=(k+\lceil m j / b\rceil) / m$. For the birational transform $E=h_{1 *}^{-1} E_{1}$, we see that $a\left(E, X, \Delta_{X}(k)\right)=(k+1) / m$. Thus $f_{1}=h_{1} \circ g_{1}: X_{1} \rightarrow X$ is a $K_{X}+\mathcal{H}_{X}$-crepant birational morphism which satisfies the condition (i).

Next we shall study the point $Q_{4}$. We know that

$$
U_{4}=\left\{\bar{x} \bar{y}+f\left(\bar{z} \bar{u}^{1 / m}, \bar{u}\right) / \bar{u}^{w_{1}(X)}=0\right\} / \mathbb{Z}_{m}(\alpha,-\alpha, 1,0) .
$$

If $l(X)=1$, then $u^{w_{1}(X)} \in f(z, u)$ and $Q_{4} \notin U_{4}$, hence $X_{1}$ is Gorenstein. If $l(X)>1$, then $Q_{4} \in U_{4}$ and we see that $\left(g_{1}^{*} \Delta_{X}(k)-\frac{1}{m} E_{1}\right)_{\mid U_{4}}=$ $-\frac{k+1}{m} \operatorname{div}_{U_{4}}(\bar{u})$. Hence, for each prime divisor $G$ over $Q_{4}$, we have

$$
\begin{aligned}
a\left(G, X, \Delta_{X}(k)\right) & =a\left(G, X_{1}, g_{1}^{*} \Delta_{X}(k)-\frac{1}{m} E_{1}\right) \\
& =a\left(G, U_{4},-\frac{k+1}{m} \operatorname{div}_{U_{4}}(\bar{u})\right) .
\end{aligned}
$$

We also see that the morphism $f_{1}: X_{1} \rightarrow X$ satisfies the condition (iii) when $l(X)>1$.

Remark 4.5. In (4.4)(iii), we have $w_{i}(U)=w_{i+1}(X)-w_{1}(X)$ for all positive integers $i$, and $l(U)=l(X)-1$.

TheOREM 4.6. Let $X, w_{i}(X), l(X), \Delta_{X}(k), \mathcal{H}_{X}$ be as in (4.1) and (4.3). Then there is a $K_{X}+\mathcal{H}_{X}$-crepant Gorenstein resolution $f: Y \rightarrow X$ such that $\mathcal{E}(f)=\bigcup_{i=1}^{m+l(X)-1} \mathcal{E}\left(f, \Delta_{X}(k),(k+i) / m\right)$ with

$$
\begin{aligned}
& \# \mathcal{E}\left(f, \Delta_{X}(k),(k+i) / m\right) \\
& = \begin{cases}w_{i}(X) & \text { if } i=1, \ldots, m-1, \\
w_{i}(X)-w_{i-m}(X)-1 & \text { if } i=m, \ldots, m+l(X)-1 .\end{cases}
\end{aligned}
$$


Proof. We shall prove this by induction on $l(X)$. We take positive integers $a, b$ such that $a+b=m w_{1}(X), a \equiv \alpha(\bmod m)$, and construct a $K_{X}+\mathcal{H}_{X}$-creapnt birational morphism $f_{1}: X_{1} \rightarrow X$ as in (4.4).

If $l(X)=1$, then $f_{1}: X_{1} \rightarrow X$ is a $K_{X}+\mathcal{H}_{X}$-crepant Gorenstein resolution of $X$ by (4.4)(ii). It follows from (4.4)(i) that $a\left(F, X, \Delta_{X}(k)\right) \geq$ $(k+1) / m$ for all $F \in \mathcal{E}\left(f_{1}\right)$ and that

$$
\begin{aligned}
& \#\left\{F \in \mathcal{E}\left(f_{1}\right) \mid a\left(F, X, \Delta_{X}(k)\right) \leq(k+i) / m\right\} \\
& \quad= \begin{cases}1+\lfloor a i / m\rfloor+\lfloor b i / m\rfloor=i w_{1}(X) & \text { if } i=1,2, \ldots, m-1, \\
m w_{1}(X)-1 & \text { if } i \geq m .\end{cases}
\end{aligned}
$$

Hence we see that $\# \mathcal{E}\left(f_{1}\right)=m w_{1}(X)-1$ with

$$
\# \mathcal{E}\left(f_{1}, \Delta_{X}(k),(k+i) / m\right)= \begin{cases}w_{1}(X) & \text { if } i=1, \ldots, m-1 \\ w_{1}(X)-1 & \text { if } i=m\end{cases}
$$

Since $w_{i}(X)=\operatorname{aw}(X)$ for all $i \geq 1$, this completes the proof when $l(X)=1$.

If $l(X)>1$, then we have $l(U)=l(X)-1$ by (4.5). Thus we can use the induction hypothesis to $U$ and get a $K_{X_{1}}+f_{1 *}^{-1} \mathcal{H}_{X}$-crepant Gorenstein resolution $f_{2}: Y \rightarrow X_{1}$ such that $\mathcal{E}\left(f_{2}\right)=\bigcup_{i=2}^{m+l(U)} \mathcal{E}\left(f_{2}, \Delta_{U}(k+1),(k+\right.$ i) $/ m$ ) with

$$
\begin{aligned}
& \# \mathcal{E}\left(f_{2}, \Delta_{U}(k+1),(k+i) / m\right) \\
& = \begin{cases}w_{i-1}(U) & \text { if } i=2, \ldots, m, \\
w_{i-1}(U)-w_{i-1-m}(U)-1 & \text { if } i=m+1, \ldots, m+l(U) .\end{cases}
\end{aligned}
$$

The composition $f=f_{1} \circ f_{2}: Y \rightarrow X$ is a $K_{X}+\mathcal{H}_{X}$-crepant Gorenstein resolution. By using computations for $\mathcal{E}\left(f_{1}\right)$ and $(4.4)(\mathrm{iii})$, we see that $\mathcal{E}(f)=\bigcup_{i=1}^{m+l(X)-1} \mathcal{E}\left(f, \Delta_{X}(k),(k+i) / m\right)$ with

$$
\begin{aligned}
& \# \mathcal{E}\left(f, \Delta_{X}(k),(k+i) / m\right) \\
& = \begin{cases}w_{1}(X) & \text { if } i=1 \\
w_{1}(X)+w_{i-1}(U)=w_{i}(X) & \text { if } i=2, \ldots, m-1 \\
\left(w_{1}(X)-1\right)+w_{m-1}(U)=w_{m}(X)-w_{0}(X)-1 & \text { if } i=m, \\
w_{i-1}(U)-w_{i-1-m}(U)-1= & w_{i}(X)-w_{i-m}(X)-1 \\
& \text { if } i=m+1, \ldots, m+l(X)-1 .\end{cases}
\end{aligned}
$$


Corollary 4.7. Let $X, w_{i}(X), l(X), \mathcal{H}_{X}$ be as in (4.1) and (4.3). Then there is a $K_{X}+\mathcal{H}_{X}$-crepant Gorenstein resolution $f: Y \rightarrow X$ such that $\# \mathcal{E}(f)=\operatorname{maw}(X)-l(X)$ with

$$
\# \mathcal{E}(f, k / m)= \begin{cases}w_{i}(X) & \text { if } i=1, \ldots, m-1 \\ w_{i}(X)-w_{i-m}(X)-1 & \text { if } i=m, \ldots, m+l(X)-1\end{cases}
$$

Proof. This follows from (4.6) by taking $k=0$.

Remark 4.8. If a general member $D_{X}$ of $\mathcal{H}_{X}$ has a rational double point of type $A_{\text {maw }(X)-1}$ at $P$, then $f$ in (4.7) induces the minimal resolution $f_{\mid D_{Y}}: D_{Y}=f_{*}^{-1} D_{X} \rightarrow D_{X}$ of $D_{X}$. For $E \in \mathcal{E}(f), E_{\mid D_{Y}}$ need not be irreducible. Our main results (3.1), (3.2) and (3.3) are proved by (4.7) when $X$ is of type $(\mathrm{cA} / m)$.

\section{$\S 5$. Terminal singularities of type $(\mathrm{cAx} / 2)$}

5.1. Let $X$ be a germ of a 3 -dimensional terminal singularity of type $(\mathrm{cAx} / 2)$. Then there is an embedding $X \hookrightarrow(x, y, z, u) / \mathbb{Z}_{2}(0,1,1,1)$ such that

$$
X=\left\{x^{2}+y^{2}+f(z, u)=0\right\} / \mathbb{Z}_{2}(0,1,1,1),
$$

where $f(z, u) \in(z, u)^{4} \mathbb{C}\{z, u\}$ is a $\mathbb{Z}_{2}$-invariant. We denote $\tau$-wt $(z)=$ $\tau$-wt $(u)=1 / 2$ and assume that $w=\tau$-wt $(f(z, u))$. It is easy to see that $2 \leq w \in \mathbb{Z}$

Let $\mathcal{H}_{X} \subseteq\left|-K_{X}\right|$ be a linear system such that a general member has only a rational double point. We remark that a general member of $\left|-K_{X}\right|$ has a rational double point of type $D_{w+2}$.

5.2. Let $X, w$ and $\mathcal{H}_{X}$ be as in (5.1).

(A) If $(f(z, u))_{\tau-w t=w}$ is not a square, then we embed $X$ as in (5.1.1) and construct a blow up $f_{1}: X_{1} \rightarrow X$ with weight $(x, y, z, u)=\frac{1}{2}(w, w+1,1,1)$ or $\frac{1}{2}(w+1, w, 1,1)$. By [Hay99, 8.4], $f_{1}$ is divisorial with discrepancy $1 / 2$ and $X_{1}$ is Gorenstein outside one point $Q$. There is an open neighborhood $Q \in U \subseteq X_{1}$ such that $U \simeq(x, z, u) / \mathbb{Z}_{w+1}(-1,1,1)$. For the exceptional divisor $E_{1}$ of $f_{1}$, we see that $E_{1 \mid U}=\operatorname{div}_{U}\left(x^{2}+(f(z, u))_{\tau-w t=w}\right)$.

(B) If $(f(z, u))_{\tau-w t=w}$ is a square, then we can write $(f(z, u))_{\tau-w t=w}=$ $-g(z, u)^{2}$. In this case, by using [Hay99, 8.6], there is another embedding 
$X \hookrightarrow(x, y, z, u) / \mathbb{Z}_{2}(0,1,1,1)$ such that

$$
X=\left\{\begin{array}{r}
\left\{x^{2}+y^{2}+2 g(z, u) x+h(z, u)=0\right\} / \mathbb{Z}_{2}(0,1,1,1) \\
\text { if } w \text { is even } \\
\left\{x^{2}+y^{2}+2 g(z, u) y+h(z, u)=0\right\} / \mathbb{Z}_{2}(0,1,1,1) \\
\text { if } w \text { is odd }
\end{array}\right.
$$

where $h(z, u)=(f(z, u))_{\tau-w t \geq w+1}$. By [Hay99, 8.8], the blow up $f_{2}: X_{2} \rightarrow$ $X$ with weight $(x, y, z, u)=\frac{1}{2}(w+2, w+1,1,1)$ (in the case $w$ is even) or $\frac{1}{2}(w+1, w+2,1,1)$ (in the case $w$ is odd) is divisorial with discrepancy $1 / 2$ and $X_{2}$ is Gorenstein outside one point $Q^{\prime}$. There is an open neighborhood $Q^{\prime} \in U^{\prime} \subseteq X_{2}$ such that $U^{\prime} \simeq(y, z, u) / \mathbb{Z}_{w+2}(-1,1,1)$. Let $E_{2}$ be the exceptional divisor of $f_{2}$, then we see that $E_{2 \mid U^{\prime}}=\operatorname{div}_{U^{\prime}}\left(y^{2}+g(z, u)+\right.$ $\left.(h(z, u))_{\tau-w t=w+1}\right)$.

TheOrem 5.3. Let $X, w$ and $\mathcal{H}_{X}$ be as in (5.1). Then there is a $K_{X}+\mathcal{H}_{X}$-crepant Gorenstein resolution $f: Y \rightarrow X$ such that,

(1) if $(f(z, u))_{\tau-w t=w}$ is not a square, $\# \mathcal{E}(f)=w+1$ with $\# \mathcal{E}(f, 1 / 2)=$ 1 and $\# \mathcal{E}(f, 1)=w$, and

(2) if $(f(z, u))_{\tau-w t=w}$ is a square, $\# \mathcal{E}(f)=w+2$ with $\# \mathcal{E}(f, 1 / 2)=2$ and $\# \mathcal{E}(f, 1)=w$.

Proof. (1) In the case $(f(z, u))_{\tau-w t=w}$ is not a square, let $f_{1}: X_{1} \rightarrow X$, $E_{1}$ and $Q \in U \subseteq X_{1}$ be as in (5.2)(A), and let $g_{1}: Y \rightarrow X_{1}$ be the economic resolution of $Q$. Then $\mathcal{E}\left(g_{1}\right)=\left\{F_{1}, \ldots, F_{w}\right\}$, where $F_{i}$ is the prime divisor over $Q$ with $a\left(F_{i}, X_{1}\right)=i /(w+1)(i=1, \ldots, w)$. By (2.10) and (2.12), we have

$$
\begin{aligned}
a\left(F_{i}, X\right) & =a\left(F_{i}, X_{1},-\frac{1}{2} E_{1}\right)=a\left(F_{i}, U,-\frac{1}{2} E_{1 \mid U}\right) \\
& =a\left(F_{i}, U,-\frac{1}{2} \operatorname{div}_{U}\left(x^{2}+(f(z, u))_{\tau-w t=w}\right)\right) \\
& =\frac{i}{w+1}+\frac{1}{2} \cdot \frac{2(w+1-i)}{w+1}=1
\end{aligned}
$$

for all $i=1, \ldots, w$. Thus $f=f_{1} \circ g_{1}: Y \rightarrow X$ is a Gorenstein resolution such that $\mathcal{E}(f)=\left\{g_{1 *}^{-1} E_{1}, F_{1}, \ldots, F_{w}\right\}$. Since $a\left(g_{1 *}^{-1} E_{1}, X\right)=1 / 2$ and since $f$ is a composition of divisorial blow ups with minimal discrepancies, we see that $f$ is $K_{X}+\mathcal{H}_{X}$-crepant and we complete the proof when $(f(z, u))_{\tau-w t=w}$ is not a square. 
(2) In the case $(f(z, u))_{\tau-w t=w}$ is a square, let $f_{2}: X_{2} \rightarrow X, E_{2}$ and $Q^{\prime} \in$ $U^{\prime} \subseteq X_{2}$ be as in (5.2)(B), and let $g_{2}: Y \rightarrow X_{2}$ be the economic resolution of $Q^{\prime}$. We see that $\mathcal{E}\left(g_{2}\right)=\left\{F_{1}, \ldots, F_{w+1}\right\}$, where $F_{i}$ is the prime divisor over $Q^{\prime}$ with $a\left(F_{i}, X_{2}\right)=i /(w+2)(i=1, \ldots, w+1)$. By (2.10) and (2.12), we see that $a\left(F_{1}, X\right)=a\left(F_{1}, X_{2},-\frac{1}{2} E_{2}\right)=a\left(F_{1}, U^{\prime},-\frac{1}{2} E_{2 \mid U^{\prime}}\right)=1 / 2$ and $a\left(F_{i}, X\right)=1$ for $i=2, \ldots, w+1$. Thus $f=f_{2} \circ g_{2}: Y \rightarrow X$ is a $K_{X}+\mathcal{H}_{X^{-}}$ crepant Gorenstein resolution with required properties.

Remark 5.4. If a general member $D_{X}$ of $\mathcal{H}_{X}$ has a rational double point of type $D_{w+2}$, then $f$ in (5.3) induces the minimal resolution $f_{\mid D_{Y}}$ : $D_{Y}=f_{*}^{-1} D_{X} \rightarrow D_{X}$ of $D_{X}$. In case (5.3)(1), $g_{1 *}^{-1} E_{1}$ is irreducible but $g_{1 *}^{-1} E_{1 \mid D_{Y}}$ has two irreducible components. Our main results (3.1), (3.2) and (3.3) is proved when $X$ is of type $(\mathrm{cAx} / 2)$.

\section{§6. Terminal singularities of type $(\mathrm{cD} / 3)$}

6.1. Let $X$ be a germ of a 3-dimensional terminal singularity of type $(\mathrm{cD} / 3)$. Then there is an embedding $X \hookrightarrow(x, y, z, u) / \mathbb{Z}_{3}(2,1,1,0)$ such that

$$
X=\left\{\begin{array}{l}
\left\{u^{2}+x^{3}+y z^{2}+y^{2} z=0\right\} / \mathbb{Z}_{3}(2,1,1,0) \\
\left\{u^{2}+x^{3}+y z^{2}+x y^{4} \lambda\left(y^{3}\right)+y^{6} \mu\left(y^{3}\right)=0\right\} / \mathbb{Z}_{3}(2,1,1,0) \\
\text { or } \\
\left\{\begin{array}{c}
u^{2}+x^{3}+y^{3}+x y z^{3} \alpha\left(z^{3}\right) \\
+x z^{4} \beta\left(z^{3}\right)+y z^{5} \gamma\left(z^{3}\right)+z^{6} \delta\left(z^{3}\right)=0
\end{array}\right\} / \mathbb{Z}_{3}(2,1,1,0)
\end{array}\right.
$$

where $\lambda(t), \mu(t), \alpha(t), \beta(t), \gamma(t), \delta(t) \in \mathbb{C}\{t\}$.

Let $\mathcal{H}_{X} \subseteq\left|-K_{X}\right|$ be a linear system such that a general member has only a rational double point. We remark that a general member of $\left|-K_{X}\right|$ has a rational double point of type $E_{6}$.

6.2. Let $X \hookrightarrow(x, y, z, u) / \mathbb{Z}_{3}(2,1,1,0)$ be as in (6.1.1) and assume that

$$
\text { the blow up } f_{1}: X_{1} \rightarrow X \text { with weight }(x, y, z, u)=\frac{1}{3}(2,4,1,3)
$$

is divisorial with discrepancy $1 / 3$.

Under the assumption (6.2.1), it follows from [Hay99, 9.4] that $X_{1}$ is Gorenstein outside one point $Q$ (the origin of the $y$-chart), which is terminal of type $(\mathrm{cAx} / 4)$. Let $E$ be the exceptional divisor of $f_{1}$. Then, by (2.10), 
for each prime divisor $F$ over $Q$ and $\mathbb{Q}$-Cartier divisor $\Delta$ on $X$, we have $a(F, X, \Delta)=a\left(F, X_{1}, f_{1}^{*} \Delta-\frac{1}{3} E\right)$.

Thus the study of terminal singularities of type $(\mathrm{cD} / 3)$ is reduced to the study of terminal singularities of type $(\mathrm{cAx} / 4)$. In this section, divisors $\Delta$ is chosen so that it will work well when we study terminal singularities of type $(\mathrm{cE} / 2)$ in Section 7.

Lemma 6.3. Let

$$
U=\left\{u^{2}+x^{3}+z^{2}+y z=0\right\} / \mathbb{Z}_{4}(2,1,1,3)
$$

be a germ of a 3-dimensional terminal singularity of type $(\mathrm{cAx} / 4)$. Let $\mathcal{H}_{U} \subseteq\left|-K_{U}\right|$ be a linear system such that a general member has only a rational double point. For $1 \neq \alpha \in \mathbb{C}$ and $k \in \mathbb{Q}$, let

$$
\Delta_{\alpha}^{\prime}(k)= \begin{cases}-\frac{k}{2} \operatorname{div}_{U}\left(y+\alpha z+A^{\prime}(x, y, z)\right)-\frac{k+2}{6} \operatorname{div}_{U}(y) & \text { if } \alpha \neq 0,1, \\ -\frac{2 k+1}{3} \operatorname{div}_{U}(y) & \text { if } \alpha=0,\end{cases}
$$

where $A^{\prime}(x, y, z) \in(x, y, z)^{2} \mathbb{C}\{x, y, z\}$ is a $\mathbb{Z}_{4}$-semi-invariant. Then there is a $K_{U}+\mathcal{H}_{U}$-crepant Gorenstein resolution $g: V \rightarrow U$ such that $\mathcal{E}(g)=$ $\left\{F_{1}, \ldots, F_{5}\right\}$ with

$$
\begin{array}{ll}
a\left(F_{1}, U, \Delta_{\alpha}^{\prime}(k)\right)=(k+2) / 6, & a\left(F_{2}, U, \Delta_{\alpha}^{\prime}(k)\right)=(k+2) / 6, \\
a\left(F_{3}, U, \Delta_{\alpha}^{\prime}(k)\right)=(k+2) / 3, & a\left(F_{4}, U, \Delta_{\alpha}^{\prime}(k)\right)=(k+2) / 2, \\
a\left(F_{5}, U, \Delta_{\alpha}^{\prime}(k)\right)=(2 k+4) / 3 . &
\end{array}
$$

Proof. Let $g_{1}: U_{1} \rightarrow U$ be the blow up with weight $(x, y, z, u)=$ $\frac{1}{4}(2,1,5,3)$. By [Hay99, 7.9], $g_{1}$ is divisorial with discrepancy $1 / 4$ and $U_{1}$ is Gorenstein outside one point $Q^{\prime}$ (which is the origin of the $z$-chart). There is an open neighborhood $Q^{\prime} \in W \subseteq U_{1}$ such that $W \simeq(x, z, u) / \mathbb{Z}_{5}(2,1,3)$. We also have

$$
\begin{aligned}
& \left(g_{1}^{*} \Delta_{\alpha}^{\prime}(k)-\frac{1}{4} F\right)_{\mid W} \\
& \quad=\left\{\begin{array}{cl}
-\frac{k}{2} \operatorname{div}_{W}\left((\alpha-1) z-u^{2}-x^{3}+A^{\prime}\left(x z^{1 / 2}, y z^{1 / 4}, z^{5 / 4}\right) / z^{1 / 4}\right) \\
-\frac{k+2}{6} \operatorname{div}_{W}\left(z+u^{2}+x^{3}\right)-\frac{k+2}{6} \operatorname{div}_{W}(z) & \text { if } \alpha \neq 0,1, \\
-\frac{2 k+1}{3} \operatorname{div}_{W}\left(z+u^{2}+x^{3}\right)-\frac{k+2}{6} \operatorname{div}_{W}(z) & \text { if } \alpha=0,
\end{array}\right.
\end{aligned}
$$

where $F$ is the exceptional divisor of $g_{1}$. We shall denote the economic resolution of $Q^{\prime}$ by $h_{1}: V \rightarrow U_{1}$. Then we have $\mathcal{E}\left(h_{1}\right)=\left\{G_{1}, \ldots, G_{4}\right\}$, 
where $G_{i}$ is the prime divisor over $Q^{\prime}$ with $a\left(G_{i}, U_{1}\right)=i / 5(i=1, \ldots, 4)$. By $(2.10)$ and (2.12), we have $a\left(G_{1}, U, \Delta_{\alpha}^{\prime}(k)\right)=a\left(G_{1}, U_{1}, g_{1}^{*} \Delta_{\alpha}^{\prime}(k)-\frac{1}{4} F\right)=$ $a\left(G_{1}, W,\left(g_{1}^{*} \Delta_{\alpha}^{\prime}(k)-\frac{1}{4} F\right)_{\mid W}\right)=(k+2) / 6$. Similarly $a\left(G_{2}, U, \Delta_{\alpha}^{\prime}(k)\right)=$ $(k+2) / 3, a\left(G_{3}, U, \Delta_{\alpha}^{\prime}(k)\right)=(k+2) / 2$ and $a\left(G_{4}, U, \Delta_{\alpha}^{\prime}(k)\right)=(2 k+4) / 3$. Since $a\left(F_{1}, U, \Delta_{\alpha}^{\prime}(k)\right)=(k+2) / 6$ for the birational transform $F_{1}=h_{1 *}^{-1} F$, we complete the proof by setting $g=g_{1} \circ h_{1}: V \rightarrow U$ and $F_{i+1}=G_{i}$ for $i=1, \ldots, 4$.

\section{TheOREM 6.4. Let}

$$
X=\left\{u^{2}+x^{3}+y z^{2}+y^{2} z=0\right\} / \mathbb{Z}_{3}(2,1,1,0)
$$

and let $\mathcal{H}_{X}$ be as in (6.1). Let $\Delta_{\alpha}(k)=-\frac{k}{2} \operatorname{div}_{X}(y+\alpha z+A(x, y, z))$, where $A(x, y, z) \in(x, y, z)^{2} \mathbb{C}\{x, y, z\}$ is a $\mathbb{Z}_{3}$-semi-invariant, $1 \neq \alpha \in \mathbb{C}$ and $k \in \mathbb{Q}$. Then there is a $K_{X}+\mathcal{H}_{X}$-crepant Gorenstein resolution $f: Y \rightarrow X$ such that $\mathcal{E}(f)=\left\{E_{1}, \ldots, E_{6}\right\}$ with

$$
\begin{aligned}
& a\left(E_{1}, X, \Delta_{\alpha}(k)\right)= \begin{cases}(k+2) / 6 & \text { if } \alpha \neq 0,1, \\
(2 k+1) / 3 & \text { if } \alpha=0,\end{cases} \\
& a\left(E_{2}, X, \Delta_{\alpha}(k)\right)=(k+2) / 6, \quad a\left(E_{3}, X, \Delta_{\alpha}(k)\right)=(k+2) / 6, \\
& a\left(E_{4}, X, \Delta_{\alpha}(k)\right)=(k+2) / 3, \quad a\left(E_{5}, X, \Delta_{\alpha}(k)\right)=(k+2) / 2 \text {, } \\
& a\left(E_{6}, X, \Delta_{\alpha}(k)\right)=(2 k+4) / 3 \text {. }
\end{aligned}
$$

In particular, we have $\# \mathcal{E}(f)=6$ with $\# \mathcal{E}(f, 1 / 3)=3, \# \mathcal{E}(f, 2 / 3)=$ $\# \mathcal{E}(f, 1)=\# \mathcal{E}(f, 4 / 3)=1$.

Proof. By [Hay99, 9.4, 9.9], the assumption (6.2.1) is satisfied. Under the notation in (6.2), there is an open neighborhood $Q \in U \subseteq X_{1}$ such that

$$
U \simeq\left\{u^{2}+x^{3}+z^{2}+y z=0\right\} / \mathbb{Z}_{4}(2,1,1,3) .
$$

Let $E$ be the exceptional divisor of $f_{1}$. Then we have

$$
\begin{aligned}
& \left(f_{1}^{*} \Delta_{\alpha}(k)-\frac{1}{3} E\right)_{\mid U} \\
& \quad= \begin{cases}-\frac{k}{2} \operatorname{div}_{U}\left(y+\alpha z+A^{\prime}(x, y, z)\right)-\frac{k+2}{6} \operatorname{div}_{U}(y) & \text { if } \alpha \neq 0,1, \\
-\frac{2 k+1}{3} \operatorname{div}_{U}(y) & \text { if } \alpha=0,\end{cases}
\end{aligned}
$$

where $A^{\prime}(x, y, z)=A\left(x y^{2 / 3}, y^{4 / 3}, y^{1 / 3} z\right) / y^{1 / 3}$. We can take a Gorenstein resolution $g_{1}: Y \rightarrow X_{1}$ with $\# \mathcal{E}\left(g_{1}\right)=5$ as in $(6.3)$. For each $F \in \mathcal{E}\left(g_{1}\right)$, 
we have $a\left(F, X, \Delta_{\alpha}(k)\right)=a\left(F, X_{1}, f_{1}^{*} \Delta_{\alpha}(k)-\frac{1}{3} E\right)=a\left(F, U,\left(f_{1}^{*} \Delta_{\alpha}(k)-\right.\right.$ $\left.\left.\frac{1}{3} E\right)_{\mid U}\right)$, and these values are obtained in (6.3). For the birational transform $E_{1}=g_{1 *}^{-1} E$, we have

$$
a\left(E_{1}, X, \Delta_{\alpha}(k)\right)= \begin{cases}(k+2) / 6 & \text { if } \alpha \neq 0,1, \\ (2 k+1) / 3 & \text { if } \alpha=0 .\end{cases}
$$

Since $f_{1}$ is a divisorial blow up with minimal discrepancy and since $g_{1}$ is $K_{X_{1}}+f_{1 *}^{-1} \mathcal{H}_{X}$-crepant, it follows from (2.8) that the composition $f=$ $f_{1} \circ g_{1}: Y \rightarrow X$ is $K_{X}+\mathcal{H}_{X}$-crepant. Therefore $f$ is a $K_{X}+\mathcal{H}_{X^{-}}$-crepant Gorenstein resolution with required properties.

LEMMA 6.5. Let

$$
U=\left\{u^{2}+x^{3}+z^{2}+x y^{4} \lambda\left(y^{4}\right)+y^{6} \mu\left(y^{4}\right)=0\right\} / \mathbb{Z}_{4}(2,1,1,3)
$$

be a germ of a 3-dimensional terminal singularity of type $(\mathrm{cAx} / 4)$, where $\lambda(t), \mu(t) \in \mathbb{C}\{t\}$, and let $\mathcal{H}_{U} \subseteq\left|-K_{U}\right|$ be a linear system such that a general member has only a rational double point. Let $\Delta_{1}^{\prime}(k)=-\frac{2 k+1}{3} \operatorname{div}_{U}(y)$, $\Delta_{2}^{\prime}(k)=-\frac{k}{2} \operatorname{div}_{U}\left(z+A^{\prime}(x, y, z)\right)-\frac{k+2}{6} \operatorname{div}_{U}(y)$, where $k \in \mathbb{Q}, A^{\prime}(x, y, z) \in$ $(x, y, z)^{2} \mathbb{C}\{x, y, z\}$ is a $\mathbb{Z}_{4}$-semi-invariant. Then there is a $K_{U}+\mathcal{H}_{U}$-crepant Gorenstein resolution $g: V \rightarrow U$ such that $\mathcal{E}(g)=\left\{F_{1}, \ldots, F_{5}\right\}$ with

$$
\begin{array}{ll}
a\left(F_{1}, U, \Delta_{1}^{\prime}(k)\right)=(k+2) / 6, & a\left(F_{2}, U, \Delta_{1}^{\prime}(k)\right)=(k+2) / 3, \\
a\left(F_{3}, U, \Delta_{1}^{\prime}(k)\right)=(k+2) / 3, & a\left(F_{4}, U, \Delta_{1}^{\prime}(k)\right)=(k+2) / 2, \\
a\left(F_{5}, U, \Delta_{1}^{\prime}(k)\right)=(2 k+4) / 3, &
\end{array}
$$

and

$$
\begin{array}{ll}
a\left(F_{1}, U, \Delta_{2}^{\prime}(k)\right)=(2 k+1) / 3, & a\left(F_{2}, U, \Delta_{2}^{\prime}(k)\right)=(5 k+4) / 6, \\
a\left(F_{3}, U, \Delta_{2}^{\prime}(k)\right)=(k+2) / 3, & a\left(F_{4}, U, \Delta_{2}^{\prime}(k)\right)=(k+2) / 2, \\
a\left(F_{5}, U, \Delta_{2}^{\prime}(k)\right)=(2 k+4) / 3 . &
\end{array}
$$

Proof. Let $g_{1}: U_{1} \rightarrow U$ be the blow up with weight $(x, y, z, u)=$ $\frac{1}{4}(2,1,5,3)$. By [Hay99, 7.4], $g_{1}$ is divisorial with discrepancy $1 / 4$ and $U_{1}$ is Gorenstein outside one point $Q^{\prime}$ (which is the origin of the $z$-chart). There is an open neighborhood $Q^{\prime} \in W \subseteq U_{1}$ such that $W \simeq(x, z, u) / \mathbb{Z}_{5}(2,1,3)$. We have an economic resolution $h_{1}: V \rightarrow U_{1}$ as in (2.12). Then $\# \mathcal{E}\left(h_{1}\right)=4$. Thus $g=g_{1} \circ h_{1}: V \rightarrow U$ is a $K_{U}+\mathcal{H}_{U}$-crepant Gorenstein resolution with $\# \mathcal{E}(g)=5$. We can compute the discrepancies of each $F \in \mathcal{E}(g)$ as in the proof of (6.3) and obtain the result. 
THEOREM 6.6. Let

$$
X=\left\{u^{2}+x^{3}+y z^{2}+x y^{4} \lambda\left(y^{3}\right)+y^{6} \mu\left(y^{3}\right)=0\right\} / \mathbb{Z}_{3}(2,1,1,0)
$$

and $\mathcal{H}_{X}$ be as in (6.1). Let $\Delta_{1}(k)=-\frac{k}{2} \operatorname{div}_{X}(y+A(x, y, z))$ and $\Delta_{2}(k)=$ $-\frac{k}{2} \operatorname{div}_{X}(z+B(x, y, z))$, where $A(x, y, z)$ and $B(x, y, z) \in(x, y, z)^{2} \mathbb{C}\{x, y, z\}$ are $\mathbb{Z}_{3}$-semi-invariants, and $k \in \mathbb{Q}$. Then there is a $K_{X}+\mathcal{H}_{X}$-crepant Gorenstein resolution $f: Y \rightarrow X$ such that $\mathcal{E}(f)=\left\{E_{1}, \ldots, E_{6}\right\}$ with

$$
\begin{array}{ll}
a\left(E_{1}, X, \Delta_{1}(k)\right)=(2 k+1) / 3, & a\left(E_{2}, X, \Delta_{1}(k)\right)=(k+2) / 6, \\
a\left(E_{3}, X, \Delta_{1}(k)\right)=(k+2) / 3, & a\left(E_{4}, X, \Delta_{1}(k)\right)=(k+2) / 3, \\
a\left(E_{5}, X, \Delta_{1}(k)\right)=(k+2) / 2, & a\left(E_{6}, X, \Delta_{1}(k)\right)=(2 k+4) / 3,
\end{array}
$$

and

$$
\begin{array}{ll}
a\left(E_{1}, X, \Delta_{2}(k)\right)=(k+2) / 6, & a\left(E_{2}, X, \Delta_{2}(k)\right)=(2 k+1) / 3, \\
a\left(E_{3}, X, \Delta_{2}(k)\right)=(5 k+4) / 6, & a\left(E_{4}, X, \Delta_{2}(k)\right)=(k+2) / 3, \\
a\left(E_{5}, X, \Delta_{2}(k)\right)=(k+2) / 2, & a\left(E_{6}, X, \Delta_{2}(k)\right)=(2 k+4) / 3 .
\end{array}
$$

In particular, we have $\# \mathcal{E}(f)=6$ with $\# \mathcal{E}(f, 1 / 3)=\# \mathcal{E}(f, 2 / 3)=2$, $\# \mathcal{E}(f, 1)=\# \mathcal{E}(f, 4 / 3)=1$.

Proof. By [Hay99, 9.4, 9.14], the assumption (6.2.1) is satisfied. Under the notation in (6.2), there is an open neighborhood $Q \in U \subseteq X_{1}$ such that

$$
U \simeq\left\{u^{2}+x^{3}+z^{2}+x y^{4} \lambda\left(y^{4}\right)+y^{6} \mu\left(y^{4}\right)=0\right\} / \mathbb{Z}_{4}(2,1,1,3) .
$$

Let $E$ be the exceptional divisor of $f_{1}$. Then we have

$$
\left(f_{1}^{*} \Delta_{j}(k)-\frac{1}{3} E\right)_{\mid U}= \begin{cases}-\frac{2 k+1}{3} \operatorname{div}_{U}(y) & \text { if } j=1, \\ -\frac{k}{2} \operatorname{div}_{U}\left(z+A^{\prime}(x, y, z)\right)-\frac{k+2}{6} \operatorname{div}_{U}(y) & \text { if } j=2,\end{cases}
$$

where $A^{\prime}(x, y, z)=A\left(x y^{2 / 3}, y^{4 / 3}, y^{1 / 3} z\right) / y^{1 / 3}$. We can take a Gorenstein resolution $g_{1}: Y \rightarrow X_{1}$ with $\# \mathcal{E}\left(g_{1}\right)=5$ as in (6.5). For each $F \in \mathcal{E}\left(g_{1}\right)$ and each $i=1,2$, we have $a\left(F, X, \Delta_{i}(k)\right)=a\left(F, X_{1}, f_{1}^{*} \Delta_{i}(k)-\frac{1}{3} E\right)=$ $a\left(F, U,\left(f_{1}^{*} \Delta_{i}(k)-\frac{1}{3} E\right)_{\mid U}\right)$, and these values are obtained in (6.5). For the birational transform $E_{1}=g_{1 *}^{-1} E$, we have $a\left(E_{1}, X, \Delta_{1}(k)\right)=(2 k+1) / 3$ and $a\left(E_{1}, X, \Delta_{2}(k)\right)=(k+2) / 6$. Therefore $f=f_{1} \circ g_{1}: Y \rightarrow X$ is a $K_{X}+\mathcal{H}_{X^{-}}$ crepant Gorenstein resolution with required properties. 
6.7. Let $X$ be a germ of a 3-dimensional terminal singularity of type $(\mathrm{cD} / 3)$, and assume there is an embedding $X \hookrightarrow(x, y, z, u) / \mathbb{Z}_{3}(2,1,1,0)$ such that

$$
X=\left\{\begin{array}{l}
u^{2}+x^{3}+y^{3}+x y z^{3} \alpha\left(z^{3}\right) \\
+x z^{4} \beta\left(z^{3}\right)+y z^{5} \gamma\left(z^{3}\right)+z^{6} \delta\left(z^{3}\right)=0
\end{array}\right\} / \mathbb{Z}_{3}(2,1,1,0),
$$

where $\alpha(t), \beta(t), \gamma(t), \delta(t) \in \mathbb{C}\{t\}$. We write $\alpha\left(z^{3}\right)=\sum_{i=0}^{\infty} \alpha_{i} z^{3 i}$ etc. as power series, and consider the following system of equations in $x$ :

$$
x^{3}+\beta_{0} x+\delta_{0}=0,3 x^{2}+\beta_{0}=0, \alpha_{0} x+\gamma_{0}=0, \beta_{1} x+\delta_{1}=0 .
$$

We shall divide the case into two subcases:

(A) (6.7.2) has no solutions in $x$. In this case we embed $X$ as in (6.7.1), then it follows from [Hay99, 9.4, 9.20] that the blow up $f_{1}: X_{1} \rightarrow X$ with weight $(x, y, z, u)=\frac{1}{3}(2,4,1,3)$ is divisorial with discrepancy $1 / 3$, hence we can use the discussion in (6.2). We also consider the $\mathbb{Q}$-Cartier divisor $\Delta(k)=-\frac{k}{2} \operatorname{div}_{X}(y+A(x, y, z))$, where $k \in \mathbb{Q}$ and $A(x, y, z) \in$ $(x, y, z)^{2} \mathbb{C}\{x, y, z\}$ is a $\mathbb{Z}_{3}$-semi-invariant.

(B) (6.7.2) has a solution $x=\nu \in \mathbb{C}$. By [Hay99, 9.23], there is another embedding $X \hookrightarrow(x, y, z, u) / \mathbb{Z}_{3}(2,1,1,0)$ such that

$$
X=\left\{\begin{array}{c}
u^{2}+x^{3}+3 \nu x^{2} z^{2}+y^{3}+x y z^{3} \alpha^{\prime}\left(z^{3}\right) \\
+x z^{7} \beta^{\prime}\left(z^{3}\right)+y z^{8} \gamma^{\prime}\left(z^{3}\right)+z^{12} \delta^{\prime}\left(z^{3}\right)=0
\end{array}\right\} / \mathbb{Z}_{3}(2,1,1,0)
$$

where $\alpha^{\prime}(t), \beta^{\prime}(t), \gamma^{\prime}(t), \delta^{\prime}(t) \in \mathbb{C}\{t\}$. We see from [Hay99, 9.5, 9.25] that the blow up $f_{2}: X_{2} \rightarrow X$ with weight $(x, y, z, u)=\frac{1}{3}(5,4,1,6)$ is divisorial with discrepancy $1 / 3$ and that $X_{2}$ is Gorenstein outside two points $Q_{1}$ and $Q_{2}$. One point $Q_{1}$ (which is the origin of the $x$-chart) has an open neighborhood $U_{1} \subseteq X_{2}$ such that $U_{1} \simeq(y, z, u) / \mathbb{Z}_{5}(4,1,1)$, and the other point $Q_{2}$ has an open neighborhood $U_{2} \subseteq X_{2}$ such that $U_{2} \simeq(x, y, z) / \mathbb{Z}_{2}(1,1,1)$.

The exceptional divisor $E_{2}$ of $f_{2}$ satisfies that $E_{2 \mid U_{1}}=\operatorname{div}_{U_{1}}(\chi(y, z, u))$, where $\chi(y, z, u)=u^{2}+3 \nu z^{2}+y^{3}+\alpha^{\prime}(0) y z^{3}+\beta^{\prime}(0) z^{7}+\gamma^{\prime}(0) y z^{8}+\delta^{\prime}(0) z^{12}$, and that $E_{2 \mid U_{2}}=\operatorname{div}_{U_{2}}(y)$.

We also consider the $\mathbb{Q}$-Cartier divisor $\Delta_{1}^{\prime}(k)=-\frac{k}{2} \operatorname{div}_{X}\left(y+A^{\prime}(x, y, z)\right)$ and $\Delta_{2}^{\prime}(k)=-\frac{k}{2} \operatorname{div}_{X}\left(z+B^{\prime}(x, y, z)\right)$, where $A^{\prime}(x, y, z)$ and $B^{\prime}(x, y, z) \in$ $(x, y, z)^{2} \mathbb{C}\{x, y, z\}$ are $\mathbb{Z}_{3}$-semi-invariants, and $k \in \mathbb{Q}$.

LEMMA 6.8. Let

$$
U=\left\{\begin{array}{l}
u^{2}+x^{3}+y^{2}+x y z^{3} \alpha\left(y z^{3}\right) \\
+x z^{4} \beta\left(y z^{3}\right)+y z^{5} \gamma\left(y z^{3}\right)+z^{6} \delta\left(y z^{3}\right)=0
\end{array}\right\} / \mathbb{Z}_{4}(2,1,1,3)
$$


be a germ of a 3-dimensional terminal singularity of type (cAx/4), where $\alpha(t), \beta(t), \gamma(t), \delta(t) \in \mathbb{C}\{t\}$, and let $\mathcal{H}_{U} \subseteq\left|-K_{U}\right|$ be a linear system such that a general member has only a rational double point. Let $\Delta^{\prime}(k)=$ $-\frac{2 k+1}{3} \operatorname{div}_{U}(y)$, where $k \in \mathbb{Q}$. Then there is a $K_{U}+\mathcal{H}_{U}$-crepant Gorenstein resolution $g: V \rightarrow U$ such that $\mathcal{E}(g)=\left\{F_{1}, \ldots, F_{5}\right\}$ with

$$
\begin{array}{ll}
a\left(F_{1}, U, \Delta^{\prime}(k)\right)=(5 k+4) / 6, & a\left(F_{2}, U, \Delta^{\prime}(k)\right)=k+1, \\
a\left(F_{3}, U, \Delta^{\prime}(k)\right)=(k+2) / 3, & a\left(F_{4}, U, \Delta^{\prime}(k)\right)=(k+2) / 2, \\
a\left(F_{5}, U, \Delta^{\prime}(k)\right)=(2 k+4) / 3 . &
\end{array}
$$

Proof. Let $g_{1}: U_{1} \rightarrow U$ be the blow up with weight $(x, y, z, u)=$ $\frac{1}{4}(2,5,1,3)$. By [Hay99, 7.4], $g_{1}$ is divisorial with discrepancy $1 / 4$ and $U_{1}$ is Gorenstein outside one point $Q^{\prime}$ (which is the origin of the $y$-chart). There is an open neighborhood $Q^{\prime} \in W \subseteq U_{1}$ such that $W \simeq(x, z, u) / \mathbb{Z}_{5}(2,1,3)$. We have an economic resolution $h_{1}: V \rightarrow U_{1}$ with $\# \mathcal{E}\left(h_{1}\right)=4$. Therefore $g=g_{1} \circ h_{1}: V \rightarrow U$ is a $K_{U}+\mathcal{H}_{U}$-crepant Gorenstein resolution with $\# \mathcal{E}(g)=5$. We can compute the discrepancies of $F \in \mathcal{E}(g)$ as in the proof of (6.3) and obtain the result.

TheOREM 6.9. Let $X, \mathcal{H}_{X}, \Delta(k), \Delta_{1}^{\prime}(k), \Delta_{2}^{\prime}(k)$ be as in (6.7). Under the notation and assumptions in (6.7), we have the following:

(1) In case $(\mathrm{A})$, there is a $K_{X}+\mathcal{H}_{X}$-crepant Gorenstein resolution $f: Y \rightarrow X$ such that $\mathcal{E}(f)=\left\{E_{1}, \ldots, E_{6}\right\}$ with

$$
\begin{array}{ll}
a\left(E_{1}, X, \Delta(k)\right)=(2 k+1) / 3, & a\left(E_{2}, X, \Delta(k)\right)=(5 k+4) / 6, \\
a\left(E_{3}, X, \Delta(k)\right)=k+1, & a\left(E_{4}, X, \Delta(k)\right)=(k+2) / 3, \\
a\left(E_{5}, X, \Delta(k)\right)=(k+2) / 2, & a\left(E_{6}, X, \Delta(k)\right)=(2 k+4) / 3 .
\end{array}
$$

In particular, we have $\# \mathcal{E}(f)=6$ with $\# \mathcal{E}(f, 1 / 3)=1, \# \mathcal{E}(f, 2 / 3)=$ $\# \mathcal{E}(f, 1)=2$ and $\# \mathcal{E}(f, 4 / 3)=1$.

(2) In case (B), there is a $K_{X}+\mathcal{H}_{X}$-crepant Gorenstein resolution $f: Y \rightarrow X$ such that $\mathcal{E}(f)=\left\{E_{1}, \ldots, E_{6}\right\}$ with

$$
\begin{array}{ll}
a\left(E_{1}, X, \Delta_{1}^{\prime}(k)\right)=(2 k+1) / 3, & a\left(E_{2}, X, \Delta_{1}^{\prime}(k)\right)=(2 k+1) / 3, \\
a\left(E_{3}, X, \Delta_{1}^{\prime}(k)\right)=(5 k+4) / 6, & a\left(E_{4}, X, \Delta_{1}^{\prime}(k)\right)=k+1, \\
a\left(E_{5}, X, \Delta_{1}^{\prime}(k)\right)=(k+2) / 2, & a\left(E_{6}, X, \Delta_{1}^{\prime}(k)\right)=(k+2) / 3
\end{array}
$$


and

$$
\begin{array}{ll}
a\left(E_{1}, X, \Delta_{2}^{\prime}(k)\right)=(k+2) / 6, & a\left(E_{2}, X, \Delta_{2}^{\prime}(k)\right)=(k+2) / 6, \\
a\left(E_{3}, X, \Delta_{2}^{\prime}(k)\right)=(k+2) / 3, & a\left(E_{4}, X, \Delta_{2}^{\prime}(k)\right)=(k+2) / 2, \\
a\left(E_{5}, X, \Delta_{2}^{\prime}(k)\right)=(k+2) / 2, & a\left(E_{6}, X, \Delta_{2}^{\prime}(k)\right)=(k+2) / 3 .
\end{array}
$$

In particular, $\# \mathcal{E}(f)=6$ with $\# \mathcal{E}(f, 1 / 3)=\# \mathcal{E}(f, 2 / 3)=\# \mathcal{E}(f, 1)=2$.

Proof. (1) By [Hay99, 9.4, 9.20], the assumption (6.2.1) is satisfied. Under the notation in (6.2), there is an open neighborhood $Q \in U \subseteq X_{1}$ such that

$$
U \simeq\left\{\begin{array}{l}
u^{2}+x^{3}+y^{2}+x y z^{3} \alpha\left(y z^{3}\right) \\
+x z^{4} \beta\left(y z^{3}\right)+y z^{5} \gamma\left(y z^{3}\right)+z^{6} \delta\left(y z^{3}\right)=0
\end{array}\right\} / \mathbb{Z}_{4}(2,1,1,3) .
$$

We also see that $\left(f_{1}^{*} \Delta(k)-\frac{1}{3} E\right)_{\mid U}=-\frac{2 k+1}{3} \operatorname{div}_{U}(y)$, where $E$ is the exceptional divisor of $f_{1}$. By (6.8), there is a Gorenstein resolution $g_{1}: Y \rightarrow$ $X_{1}$ with $\# \mathcal{E}\left(g_{1}\right)=5$. For each $F \in \mathcal{E}\left(g_{1}\right)$, we have $a(F, X, \Delta(k))=$ $a\left(F, X_{1}, f_{1}^{*} \Delta(k)-\frac{1}{3} E\right)=a\left(F, U,\left(f_{1}^{*} \Delta(k)-\frac{1}{3} E\right)_{\mid U}\right)$ by $(2.10)$, and these values are obtained in (6.8). For the birational transform $E_{1}=g_{1 *}^{-1} E$, we have $a\left(E_{1}, X, \Delta(k)\right)=(2 k+1) / 3$. Therefore $f=f_{1} \circ g_{1}: Y \rightarrow X$ is a $K_{X}+\mathcal{H}_{X}$-crepant Gorenstein resolution with required properties.

(2) Let $f_{2}: X_{2} \rightarrow X, E_{2}, Q_{1} \in U_{1} \subseteq X_{2}, Q_{2} \in U_{2} \subseteq X_{2}$ be as in (6.7)(B). We shall denote the economic resolution of $Q_{1}$ and $Q_{2}$ by $g_{2}: Y \rightarrow$ $X_{2}$. Then $\mathcal{E}\left(g_{2}\right)=\left\{F_{1}, \ldots, F_{4}, G\right\}$, where $F_{i}$ (resp. $G$ ) is the prime divisor over $Q_{1}$ (resp. $\left.Q_{2}\right)$ with $a\left(F_{i}, X_{2}\right)=i / 5(i=1, \ldots, 4)\left(\operatorname{resp} . a\left(G, X_{2}\right)=\right.$ $1 / 2)$.

We first study $a\left(F_{i}, X, \Delta_{j}^{\prime}(k)\right)(i=1, \ldots, 4, j=1,2)$. We have

$$
\begin{aligned}
& \left(f_{2}^{*} \Delta_{j}^{\prime}(k)-\frac{1}{3} E_{2}\right)_{\mid U_{1}} \\
& \quad= \begin{cases}-\frac{k}{2} \operatorname{div}_{U_{1}}\left(y+A_{1}^{\prime}(y, z, u)\right)-\frac{2 k+1}{3} \operatorname{div}_{U_{1}}(\chi(y, z, u)) & \text { if } j=1, \\
-\frac{k}{2} \operatorname{div}_{U_{1}}\left(z+B_{1}^{\prime}(y, z, u)\right)-\frac{k+2}{6} \operatorname{div}_{U_{1}}(\chi(y, z, u)) & \text { if } j=2,\end{cases}
\end{aligned}
$$

where $A_{1}^{\prime}(y, z, u), B_{1}^{\prime}(y, z, u) \in(y, z, u)^{2} \mathbb{C}\{y, z, u\}$ are $\mathbb{Z}_{5}$-semi-invariants. By $(2.10)$, we see that $a\left(F_{1}, X, \Delta_{1}^{\prime}(k)\right)=a\left(F_{1}, U_{1},\left(f_{2}^{*} \Delta_{1}^{\prime}(k)-\frac{1}{3} E_{2}\right) \mid U_{1}\right)=$ $(2 k+1) / 3$. Similarly, we obtain values of $a\left(F_{i}, X, \Delta_{j}^{\prime}(k)\right)$ for each $i=$ $1, \ldots, 4, j=1,2$. These correspond to $a\left(E_{i+1}, X, \Delta_{j}^{\prime}(k)\right)$ respectively in the theorem. 
Next we study $a\left(G, X, \Delta_{j}^{\prime}(k)\right)$ for $j=1,2$. We have

$$
\begin{aligned}
& \left(f_{2}^{*} \Delta_{j}^{\prime}(k)-\frac{1}{3} E_{2}\right)_{\mid U_{2}} \\
& \quad= \begin{cases}-\frac{2 k+1}{3} \operatorname{div}_{U_{2}}(y) & \text { if } j=1, \\
-\frac{k}{2} \operatorname{div}_{U_{2}}\left(z+B_{2}^{\prime}(x, y, z)\right)-\frac{k+2}{6} \operatorname{div}_{U_{2}}(y) & \text { if } j=2,\end{cases}
\end{aligned}
$$

for some $\mathbb{Z}_{2}$-semi-invariant $B_{2}^{\prime}(x, y, z) \in(x, y, z)^{2} \mathbb{C}\{z, y, z\}$. Hence, by (2.10), we see that $a\left(G, X, \Delta_{1}^{\prime}(k)\right)=a\left(G, X, \Delta_{2}^{\prime}(k)\right)=(k+2) / 3$. Therefore we complete the proof by taking $f=f_{2} \circ g_{2}: Y \rightarrow X$.

Remark 6.10. Thus (6.4), (6.6) and (6.9) prove our main results (3.1), (3.2) and (3.3) when $X$ is of type (cD/3). Moreover if $D_{X} \in \mathcal{H}_{X}$ has only a rational double point of type $E_{6}$, then $f$ in (6.4), (6.6) and (6.9) induces the minimal resolution of $D_{X}$.

\section{$\S 7$. Terminal singularities of type $(\mathrm{cE} / 2)$}

7.1. Let $X$ be a germ of a 3-dimensional terminal singularity of type $(\mathrm{cE} / 2)$. Then there is an embedding $X \hookrightarrow(x, y, z, u) / \mathbb{Z}_{2}(0,1,1,1)$ such that

$$
X=\left\{u^{2}+x^{3}+g(y, z) x+h(y, z)=0\right\} / \mathbb{Z}_{2}(0,1,1,1)
$$

where $g(y, z), h(y, z) \in(y, z)^{4} \mathbb{C}\{y, z\}$ are $\mathbb{Z}_{2}$-invariants and the degree 4 part $h_{\operatorname{deg} 4}(y, z)$ of $h(y, z)$ is non-zero.

We denote by $\mathcal{H}_{X}$ a linear system $\subseteq\left|-K_{X}\right|$ such that a general member has only a rational double point, which is of type $E_{7}$.

7.2. Let $X \hookrightarrow(x, y, z, u) / \mathbb{Z}_{2}(0,1,1,1)$ be as in (7.1.1) and assume that $z^{4} \notin h(y, z)$ and that

the blow up $f_{1}: X_{1} \rightarrow X$ with weight $(x, y, z, u)=\frac{1}{2}(2,3,1,3)$

is divisorial with discrepancy $1 / 2$.

It follows from [Hay99, 10.4] that $X_{1}$ is Gorenstein outside one point $Q$ (which is the origin of the $y$-chart) and that there is an open neighborhood $Q \in U \subseteq X_{1}$ such that

$$
U \simeq\left\{u^{2}+x^{3}+g\left(y^{3 / 2}, y^{1 / 2} z\right) / y^{2} \cdot x+h\left(y^{3 / 2}, y^{1 / 2} z\right) / y^{3}=0\right\} / \mathbb{Z}_{3}(2,1,1,0) .
$$

Since $h_{\operatorname{deg} 4}(y, z)$ is non-zero, this is terminal of type $(\mathrm{cD} / 3)$. 
Let $E_{1}$ be the exceptional divisor of $f_{1}$. By (2.10), we see that $a(F, X)=$ $a\left(F, X_{1},-\frac{1}{2} E_{1}\right)=a\left(F, U,-\frac{1}{2} \operatorname{div}_{U}(y)\right)$ for each prime divisor $F$ over $Q$.

Thus the study of terminal singularities of type $(\mathrm{cE} / 2)$ is reduced to the study of terminal singularities of type $(\mathrm{cD} / 3)$, which is already done in Section 6.

We shall study terminal singularities of type $(\mathrm{cE} / 2)$ by dividing into several cases as in [Hay99, §10]. If $h_{\operatorname{deg} 4}(y, z)$ does not have a triple or a quadruple factor, the assumption (7.2.1) is satisfied by [Hay99, 10.4] and we can apply the discussion in (7.2).

TheOrem 7.3. Let $X, \mathcal{H}_{X}$ be as in (7.1) and assume that $h_{\operatorname{deg} 4}(y, z)$ has four distinct factors. Then there is a $K_{X}+\mathcal{H}_{X}$-crepant Gorenstein resolution $f: Y \rightarrow X$ such that $\# \mathcal{E}(f)=7$ with $\# \mathcal{E}(f, 1 / 2)=4$ and $\# \mathcal{E}(f, 1)=\# \mathcal{E}(f, 3 / 2)=\# \mathcal{E}(f, 2)=1$.

Proof. By a linear transformation in $y$ and $z$, we may assume that $h_{\operatorname{deg} 4}(y, z)=y z\left(y+\lambda_{1} z\right)\left(y+\lambda_{2} z\right)$ for some $\lambda_{1}, \lambda_{2} \in \mathbb{C}$ with $\lambda_{1}, \lambda_{2} \neq 0$, $\lambda_{1} \neq \lambda_{2}$. By [Hay99, 10.4, 10.11], the assumption (7.2.1) is satisfied. Under the notation in $(7.2)$,

$$
U \simeq\left\{\begin{array}{c}
u^{2}+x^{3}+z\left(y+\lambda_{1} z\right)\left(y+\lambda_{2} z\right) \\
+(\text { terms of degree } \geq 4)=0
\end{array}\right\} / \mathbb{Z}_{3}(2,1,1,0),
$$

and this is isomorphic to

$$
V=\left\{u^{2}+x^{3}+y z^{2}+y^{2} z=0\right\} / \mathbb{Z}_{3}(2,1,1,0) .
$$

Furthermore, we see that $\left(U,-\frac{1}{2} E_{1 \mid U}\right) \simeq\left(V,-\frac{1}{2} \operatorname{div}_{V}(y+\alpha z+A(x, y, z))\right)$ for some $\alpha \in \mathbb{C}$ with $\alpha \neq 0,1$ and some $\mathbb{Z}_{3}$-semi-invariant $A(x, y, z) \in$ $(x, y, z)^{2} \mathbb{C}\{x, y, z\}$. By (6.4), there is a $K_{X_{1}}+f_{1 *}^{-1} \mathcal{H}_{X}$-crepant Gorenstein resolution $g_{1}: Y \rightarrow X_{1}$ with $\# \mathcal{E}\left(g_{1}\right)=6$. For each $F \in \mathcal{E}\left(g_{1}\right)$, we have $a(F, X)=a\left(F, X_{1},-\frac{1}{2} E_{1}\right)=a\left(F, V,-\frac{1}{2} \operatorname{div}_{V}(y+\alpha z+A(x, y, z))\right)$, and these values are obtained in (6.4). Since $a\left(g_{1 *}^{-1} E_{1}, X\right)=1 / 2$, we complete the proof by taking $f=f_{1} \circ g_{1}: Y \rightarrow X$.

TheOREM 7.4. Let $X, \mathcal{H}_{X}$ be as in (7.1) and assume that $h_{\operatorname{deg} 4}(y, z)$ has one double and two single factors. Then there is a $K_{X}+\mathcal{H}_{X}$-crepant Gorenstein resolution $f: Y \rightarrow X$ such that $\# \mathcal{E}(f)=7$ with $\# \mathcal{E}(f, 1 / 2)=3$, $\# \mathcal{E}(f, 1)=2$ and $\# \mathcal{E}(f, 3 / 2)=\# \mathcal{E}(f, 2)=1$. 
Proof. We may assume that $h_{\operatorname{deg} 4}(y, z)=y^{2} z(y+z)$. By [Hay99, 10.4, 10.17], the assumption (7.2.1) is satisfied. Under the notation in (7.2), $U$ is isomorphic to

$$
V=\left\{u^{2}+x^{3}+y z^{2}+y^{2} z=0\right\} / \mathbb{Z}_{3}(2,1,1,0) .
$$

We also have $\left(U,-\frac{1}{2} E_{1 \mid U}\right) \simeq\left(V,-\frac{1}{2} \operatorname{div}_{V}(y+A(x, y, z))\right)$ for some $\mathbb{Z}_{3}$-semiinvariant $A(x, y, z) \in(x, y, z)^{2} \mathbb{C}\{x, y, z\}$. By (6.4), there is a $K_{X_{1}}+f_{1 *}^{-1} \mathcal{H}_{X^{-}}$ crepant Gorenstein resolution $g_{1}: Y \rightarrow X_{1}$ with $\# \mathcal{E}\left(g_{1}\right)=6$. For each $F \in \mathcal{E}\left(g_{1}\right)$, we have $a(F, X)=a\left(F, X_{1},-\frac{1}{2} E_{1}\right)=a\left(F, V,-\frac{1}{2} \operatorname{div}_{V}(y+\right.$ $A(x, y, z)))$, and these values are obtained in (6.4) by setting $\alpha=0$ and $k=1$. Thus it suffices to take $f=f_{1} \circ g_{1}: Y \rightarrow X$.

Theorem 7.5. Let $X, \mathcal{H}_{X}$ be as in (7.1) and assume that $h_{\operatorname{deg} 4}(y, z)$ has two double factors. Then there is a $K_{X}+\mathcal{H}_{X}$-crepant Gorenstein resolution $f: Y \rightarrow X$ such that $\# \mathcal{E}(f)=7$ with $\# \mathcal{E}(f, 1 / 2)=2, \# \mathcal{E}(f, 1)=3$ and $\# \mathcal{E}(f, 3 / 2)=\# \mathcal{E}(f, 2)=1$.

Proof. We may assume that $h_{\operatorname{deg} 4}(y, z)=y^{2} z^{2}$. By [Hay99, 10.4, 10.22], the assumption (7.2.1) is satisfied. Under the notation in (7.2), $U$ is isomorphic to

$$
V=\left\{u^{2}+x^{3}+y z^{2}+x y^{4} \lambda\left(y^{3}\right)+y^{6} \mu\left(y^{3}\right)=0\right\} / \mathbb{Z}_{3}(2,1,1,0)
$$

for some $\lambda(t), \mu(t) \in \mathbb{C}\{t\}$, and $\left(U,-\frac{1}{2} E_{1 \mid U}\right) \simeq\left(V,-\frac{1}{2} \operatorname{div}_{V}(y+A(x, y, z))\right)$ for some $\mathbb{Z}_{3}$-semi-invariant $A(x, y, z) \in(x, y, z)^{2} \mathbb{C}\{x, y, z\}$. By using (6.6), there is a $K_{X_{1}}+f_{1 *}^{-1} \mathcal{H}_{X}$-crepant Gorenstein resolution $g_{1}: Y \rightarrow X_{1}$ with $\# \mathcal{E}\left(g_{1}\right)=6$. For each $F \in \mathcal{E}\left(g_{1}\right)$, we have $a(F, X)=a\left(F, V,-\frac{1}{2} \operatorname{div}_{V}(y+\right.$ $A(x, y, z)))$, and these values are obtained in (6.6). Therefore $f=f_{1} \circ$ $g_{1}: Y \rightarrow X$ is a $K_{X}+\mathcal{H}_{X}$-crepant Gorenstein resolution with required properties.

7.6. Next we shall study the case where $h_{\operatorname{deg} 4}(y, z)$ has a triple factor and a single factor. We may assume that $h_{\operatorname{deg} 4}(y, z)=y^{3} z$. We write $g(y, z)=\sum_{p, q} a_{p q} y^{p} z^{q}, h(y, z)=\sum_{p, q} b_{p q} y^{p} z^{q}$ as power series, and consider the following system of equations in $x$ :

$$
x^{3}+a_{04} x+b_{06}=0,3 x^{2}+a_{04}=0, a_{13} x+b_{15}=0, a_{06} x+b_{08}=0 .
$$

We shall divide into two subcases: 
(A) (7.6.1) has no solutions in $x$. In this case, the assumption (7.2.1) is satisfied by [Hay99, 10.4, 10.28], hence we can use the discussion in (7.2).

(B) (7.6.1) has a solution $x=\nu \in \mathbb{C}$. By [Hay99, 10.31], there is another embedding $X \hookrightarrow(x, y, z, u) / \mathbb{Z}_{2}(0,1,1,1)$ such that

$$
X=\left\{u^{2}+x^{3}+3 \nu x^{2} z^{2}+g_{1}(y, z) x+h_{1}(y, z)=0\right\} / \mathbb{Z}_{2}(0,1,1,1)
$$

where $\tau-w t\left(g_{1}(y, z)\right) \geq 3, \tau-w t\left(h_{1}(y, z)\right) \geq 5$ when we set $\tau$-wt $(y, z)=$ $(3 / 2,1 / 2)$, and $y^{3} z \in h_{1}(y, z)$. In this case we shall use the blow up $f_{2}$ : $X_{2} \rightarrow X$ with weight $(x, y, z, u)=\frac{1}{2}(2,1,3,3)$. This is divisorial with discrepancy $1 / 2$, and in particular this is $K_{X}+\mathcal{H}_{X}$-crepant.

TheOREm 7.7. Let $X, \mathcal{H}_{X}$ be as in (7.1) and assume that $h_{\operatorname{deg} 4}(y, z)$ has a triple factor and a single factor. Under the notation and assumptions in (7.6), there is a $K_{X}+\mathcal{H}_{X}$-crepant Gorenstein resolution $f: Y \rightarrow X$ such that $\# \mathcal{E}(f)=7$ with

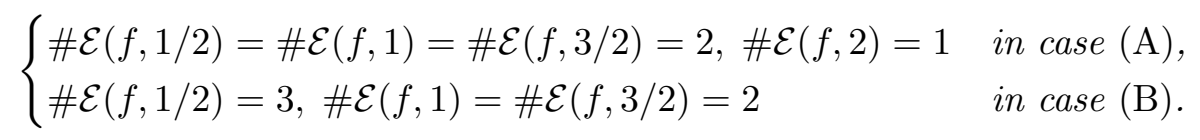

Proof. (1) In case (A), we can use the discussion in (7.2) as in (7.6)(A). Under the notation in (7.2), $U$ is isomorphic to

$$
V=\left\{u^{2}+x^{3}+y z^{2}+x y^{4} \lambda\left(y^{3}\right)+y^{6} \mu\left(y^{3}\right)=0\right\} / \mathbb{Z}_{3}(2,1,1,0),
$$

for some $\lambda(t), \mu(t) \in \mathbb{C}\{t\}$, and $\left(U,-\frac{1}{2} E_{1 \mid U}\right) \simeq\left(V,-\frac{1}{2} \operatorname{div}_{V}(z+B(x, y, z))\right)$ for some $\mathbb{Z}_{3}$-semi-invariant $B(x, y, z) \in(x, y, z)^{2} \mathbb{C}\{x, y, z\}$. By using (6.6), there is a $K_{X_{1}}+f_{1 *}^{-1} \mathcal{H}_{X}$-crepant Gorenstein resolution $g_{1}: Y \rightarrow X_{1}$ with $\# \mathcal{E}\left(g_{1}\right)=6$. For each $F \in \mathcal{E}\left(g_{1}\right)$, we have $a(F, X)=a\left(F, V,-\frac{1}{2} \operatorname{div}_{V}(z+\right.$ $B(x, y, z)))$, and these values are obtained in (6.6). Therefore we complete the proof by taking $f=f_{1} \circ g_{1}: Y \rightarrow X$.

(2) Next we treat case (B). Under the notation in $(7.6)(B)$, we see that $X_{2}$ is Gorenstein outside one point $Q^{\prime}$ (which is the origin of the $z$-chart), and there is an open neighborhood $Q^{\prime} \in U^{\prime} \subseteq X_{2}$ such that

$$
U^{\prime} \simeq\left\{\begin{array}{c}
u^{2}+x^{3}+3 \nu x^{2} z^{2}+g_{1}\left(y z^{1 / 2}, z^{3 / 2}\right) / z^{2} \cdot x \\
+h_{1}\left(y z^{1 / 2}, z^{3 / 2}\right) / z^{3}=0
\end{array}\right\} / \mathbb{Z}_{3}(2,1,1,0),
$$

which is isomorphic to

$$
V^{\prime}=\left\{\begin{array}{c}
u^{2}+x^{3}+3 \nu x^{2} z^{2}+y^{3}+x y z^{3} \alpha^{\prime}\left(z^{3}\right) \\
+x z^{7} \beta^{\prime}\left(z^{3}\right)+y z^{8} \gamma^{\prime}\left(z^{3}\right)+z^{12} \delta^{\prime}\left(z^{3}\right)=0
\end{array}\right\} / \mathbb{Z}_{3}(2,1,1,0)
$$


for some $\alpha^{\prime}(t), \beta^{\prime}(t), \gamma^{\prime}(t), \delta^{\prime}(t) \in \mathbb{C}\{t\}$. Let $E_{2}$ be the exceptional divisor of $f_{2}$. Then $\left(U^{\prime},-\frac{1}{2} E_{2 \mid U^{\prime}}\right) \simeq\left(V^{\prime},-\frac{1}{2} \operatorname{div}_{V^{\prime}}\left(z+B^{\prime}(x, y, z)\right)\right)$ for some $\mathbb{Z}_{3^{-}}$ semi-invariant $B^{\prime}(x, y, z) \in(x, y, z)^{2} \mathbb{C}\{x, y, z\}$. By (6.9), we know that there is a $K_{X_{2}}+f_{2 *}^{-1} \mathcal{H}_{X}$-crepant Gorenstein resolution $g_{2}: Y \rightarrow X_{2}$ with $\# \mathcal{E}\left(g_{2}\right)=6$. For each $F \in \mathcal{E}\left(g_{2}\right)$, we have $a(F, X)=a\left(F, X_{2},-\frac{1}{2} E_{2}\right)=$ $a\left(F, V^{\prime},-\frac{1}{2} \operatorname{div}_{V^{\prime}}\left(z+B^{\prime}(x, y, z)\right)\right)$, and these values are obtained in (6.9). Therefore $f=f_{2} \circ g_{2}: Y \rightarrow X$ is a $K_{X}+\mathcal{H}_{X}$-crepant Gorenstein resolution with required properties.

7.8. Lastly we shall study the case where $h_{\operatorname{deg} 4}(y, z)$ has a quadruple factor. We may assume that $h_{\operatorname{deg} 4}(y, z)=y^{4}$. As in (7.6), we write $g(y, z)=\sum_{p, q} a_{p q} y^{p} z^{q}, h(y, z)=\sum_{p, q} b_{p q} y^{p} z^{q}$ as power series, and consider the following two systems of equations in $x$ :

$$
x^{3}+a_{04} x+b_{06}=0,3 x^{2}+a_{04}=0, a_{13} x+b_{15}=0,
$$

and

$(7.8 .2) x^{3}+a_{04} x+b_{06}=0,3 x^{2}+a_{04}=0, a_{13} x+b_{15}=0, a_{06} x+b_{08}=0$.

We shall divide the case into six subcases:

(A) (7.8.1) has no solutions in $x$. In this case, the assumption (7.2.1) is satisfied by [Hay99, 10.4, 10.41], hence we can use the discussion in (7.2).

(B) (7.8.1) has a solution but (7.8.2) has no solutions. In this case, the assumption (7.2.1) is satisfied and we can use the discussion in (7.2). However the situation is slightly different from case (A).

If (7.8.2) has a solution $x=\nu \in \mathbb{C}$, then it follows from [Hay99, 10.44] that there is another embedding $X \hookrightarrow(x, y, z, u) / \mathbb{Z}_{2}(0,1,1,1)$ such that

$$
X=\left\{u^{2}+x^{3}+3 \nu x^{2} z^{2}+g_{1}(y, z) x+h_{1}(y, z)=0\right\} / \mathbb{Z}_{2}(0,1,1,1)
$$

where $\tau$-wt $\left(g_{1}(y, z)\right) \geq 3, \tau$-wt $\left(h_{1}(y, z)\right) \geq 5$ when we set $\tau$-wt $(y, z)=$ $(3 / 2,1 / 2)$, and $y^{4} \in h_{1}(y, z)$. We next consider the following weighted homogeneous polynomial:

$$
\Phi=u^{2}+3 \nu x^{2} z^{2}+\left(g_{1}(y, z)\right)_{\tau-w t=3} \cdot x+\left(h_{1}(y, z)\right)_{\tau-w t=5} .
$$

(C) $\{\Phi=0\} \subseteq \mathbb{P}(4,3,1,5)$ is irreducible and reduced. We embed $X$ as in (7.8.3) and construct a blow up $f_{2}: X_{2} \rightarrow X$ with weight $(x, y, z, u)=$ $\frac{1}{2}(4,3,1,5)$. By [Hay99, 10.5, 10.47], $f_{2}$ is divisorial with discrepancy $1 / 2$ 
and $X_{2}$ is Gorenstein outside two points $Q_{1}$ and $Q_{2}$ (which are the origins of the $x$-chart and the $y$-chart respectively). There is an open neighborhood $Q_{1} \in U_{1} \subseteq X_{2}$ (resp. $\left.Q_{2} \in U_{2} \subseteq X_{2}\right)$ such that $U_{1} \simeq(y, z, u) / \mathbb{Z}_{4}(3,1,1)$ (resp. $\left.U_{2} \simeq(x, z, u) / \mathbb{Z}_{3}(1,1,2)\right)$. Moreover we have $E_{2 \mid U_{1}}=\operatorname{div}_{U_{1}}\left(u^{2}+\right.$ $\left.3 \nu z^{2}+\left(g_{1}(y, z)\right)_{\tau-w t=3} \cdot x+\left(h_{1}(y, z)\right)_{\tau-w t=5}\right)$ and $E_{2 \mid U_{2}}=\operatorname{div}_{U_{2}}\left(u^{2}+3 \nu x^{2} z^{2}+\right.$ $\left.\left(g_{1}(1, z)\right)_{\tau-w t=3} \cdot x+\left(h_{1}(1, z)\right)_{\tau-w t=5}\right)$, where $E_{2}$ is the exceptional divisor of $f_{2}$.

If $\{\Phi=0\} \subseteq \mathbb{P}(4,3,1,5)$ is reducible or non-reduced, then we see from [Hay99, 10.51] that there is an embedding $X \hookrightarrow(x, y, z, u) / \mathbb{Z}_{2}(0,1,1,1)$ such that

$$
X=\left\{\begin{array}{c}
u^{2}+2\left(\alpha x z+\beta y z^{2}+\gamma z^{5}\right) u \\
+x^{3}+g_{2}(y, z) x+h_{2}(y, z)=0
\end{array}\right\} / \mathbb{Z}_{2}(0,1,1,1)
$$

where $\alpha, \beta, \gamma \in \mathbb{C}$ and $\tau-w t\left(g_{2}(y, z)\right) \geq 4, \tau-w t\left(h_{2}(y, z)\right) \geq 6$ when we set $\tau-w t(y, z)=(3 / 2,1 / 2)$, and $y^{4} \in h_{2}(y, z)$.

(D) $(\alpha, \beta, \gamma) \neq(0,0,0)$. We embed $X$ as in (7.8.5) and construct a blow up $f_{3}: X_{3} \rightarrow X$ with weight $(x, y, z, u)=\frac{1}{2}(4,3,1,7)$. By [Hay99, $10.6,10.54], f_{3}$ is divisorial with discrepancy $1 / 2, X_{3}$ is Gorenstein outside one point $Q$ (which is the origin of the $u$-chart), and there is an open neighborhood $Q \in U \subseteq X_{3}$ such that $U \simeq(x, y, z) / \mathbb{Z}_{7}(4,3,1)$ with $E_{3 \mid U}=$ $\operatorname{div}_{U}\left(2\left(\alpha x z+\beta y z^{2}+\gamma z^{5}\right)+x^{3}+\left(g_{2}(y, z)\right)_{\tau-w t=4} \cdot x+\left(h_{2}(y, z)\right)_{\tau-w t=6}\right)$, where $E_{3}$ is the exceptional divisor of $f_{3}$.

(E) $(\alpha, \beta, \gamma)=(0,0,0)$ and the blow up $f_{3}: X_{3} \rightarrow X$ with weight $(x, y, z, u)=\frac{1}{2}(4,3,1,7)$ is divisorial with discrepancy $1 / 2$. By [Hay99, $10.6,10.61]$, the non-Gorenstein point of $X_{3}$ is unique and it has the same properties as (D).

(F) $(\alpha, \beta, \gamma)=(0,0,0)$ and the blow up $f_{3}: X_{3} \rightarrow X$ with weight $(x, y, z, u)=\frac{1}{2}(4,3,1,7)$ is not divisorial with discrepancy $1 / 2$. By [Hay99, 10.65], we can change the embedding $X \hookrightarrow(x, y, z, u) / \mathbb{Z}_{2}(0,1,1,1)$ and get

$$
X=\left\{u^{2}+x^{3}+g_{3}(y, z) x+h_{3}(y, z)=0\right\} / \mathbb{Z}_{2}(0,1,1,1),
$$

where $\tau^{\prime}-w t\left(g_{3}(y, z)\right) \geq 6, \tau^{\prime}-w t\left(h_{3}(y, z)\right) \geq 9$ when we set $\tau^{\prime}-w t(y, z)=$ $(5 / 2,1 / 2)$, and $y^{4} \in h_{3}(y, z)$. It follows from [Hay99, 10.7, 10.67] that the blow up $f_{4}: X_{4} \rightarrow X$ with weight $(x, y, z, u)=\frac{1}{2}(6,5,1,9)$ is divisorial with discrepancy $1 / 2, X_{4}$ is Gorenstein outside two points $Q_{1}$ and $Q_{2}\left(Q_{1}\right.$ is the origin of the $y$-chart and $Q_{2}$ lies on the $x$-chart and the $u$-chart), and there is an open neighborhood $Q_{1} \in U_{1} \subseteq X_{4}$ (resp. $Q_{2} \in U_{2} \subseteq X_{4}$ ) such 
that $U_{1} \simeq(x, z, u) / \mathbb{Z}_{5}(1,1,4)$ (resp. $\left.U_{2} \simeq(x, y, z) / \mathbb{Z}_{3}(1,2,1)\right)$. Moreover we have $E_{4 \mid U_{1}}=\operatorname{div}_{U_{1}}\left(u^{2}+x^{3}+\left(g_{3}(1, z)\right)_{\tau^{\prime}-w t=6} \cdot x+\left(h_{3}(y, z)\right)_{\tau^{\prime}-w t=9}\right)$ and $E_{4 \mid U_{2}}=\operatorname{div}_{U_{2}}(x)$ for the exceptional divisor $E_{4}$ of $f_{4}$.

THEOREM 7.9. Let $X, \mathcal{H}_{X}$ be as in (7.1) and assume that $h_{\operatorname{deg} 4}(y, z)$ has a quadruple factor. Then, under the notation and assumptions in (7.8), there is a $K_{X}+\mathcal{H}_{X}$-crepant Gorenstein resolution $f: Y \rightarrow X$ such that $\# \mathcal{E}(f)=6$ in case $(\mathrm{C})$ and $\# \mathcal{E}(f)=7$ in other cases. More precisely,

$$
\begin{cases}\# \mathcal{E}(f, 1 / 2)=1, \# \mathcal{E}(f, 1)=\# \mathcal{E}(f, 3 / 2)=\# \mathcal{E}(f, 2)=2 & \text { in case }(\mathrm{A}), \\ \# \mathcal{E}(f, 1 / 2)=1, \# \mathcal{E}(f, 1)=3, \# \mathcal{E}(f, 3 / 2)=2, \# \mathcal{E}(f, 2)=1 \\ \# \mathcal{E}(f, 1 / 2)=2, \# \mathcal{E}(f, 1)=3, \# \mathcal{E}(f, 3 / 2)=1 & \text { in case }(\mathrm{B}), \\ \# \mathcal{E}(f, 1 / 2)=\# \mathcal{E}(f, 1)=3, \# \mathcal{E}(f, 3 / 2)=1 & \text { in case }(\mathrm{C}), \\ \# \mathcal{E}(f, 1 / 2)=2, \# \mathcal{E}(f, 1)=4, \# \mathcal{E}(f, 3 / 2)=1 & \text { in case }(\mathrm{D}), \\ \# \mathcal{E}(f, 1 / 2)=3, \# \mathcal{E}(f, 1)=4 & \text { in case }(\mathrm{F}) .\end{cases}
$$

Proof. (1) In case (A), we can use the discussion in (7.2). Under the notation in (7.2), $U$ is isomorphic to

$$
V=\left\{\begin{array}{l}
u^{2}+x^{3}+y^{3}+x y z^{3} \alpha\left(z^{3}\right) \\
+x z^{4} \beta\left(z^{3}\right)+y z^{5} \gamma\left(z^{3}\right)+z^{6} \delta\left(z^{3}\right)=0
\end{array}\right\} / \mathbb{Z}_{3}(2,1,1,0)
$$

for some $\alpha(t), \beta(t), \gamma(t), \delta(t) \in \mathbb{C}\{t\}$. Since (7.8.1) has no solutions, we are in the situation of $(6.7)(\mathrm{A})$. We also have $\left(U,-\frac{1}{2} E_{1 \mid U}\right) \simeq\left(V,-\frac{1}{2} \operatorname{div}_{V}(y+\right.$ $A(x, y, z)))$ for some $\mathbb{Z}_{3}$-semi-invariant $A(x, y, z) \in(x, y, z)^{2} \mathbb{C}\{x, y, z\}$. By (6.9), there is a $K_{X_{1}}+f_{1 *}^{-1} \mathcal{H}_{X}$-crepant Gorenstein resolution $g_{1}: Y \rightarrow X_{1}$ with $\# \mathcal{E}\left(g_{1}\right)=6$. For each $F \in \mathcal{E}\left(g_{1}\right)$, we have $a(F, X)=a\left(F, X_{1},-\frac{1}{2} E_{1}\right)=$ $a\left(F, V,-\frac{1}{2} \operatorname{div}_{V}(y+A(x, y, z))\right)$, and these values are obtained in (6.9) by setting $k=1$. Since $a\left(g_{1 *}^{-1} E_{1}, X\right)=1 / 2$, we complete the proof by taking $f=f_{1} \circ g_{1}: Y \rightarrow X$.

(2) In case (B), we again use the discussion in (7.2). Under the notation in (7.2), $U$ is isomorphic to $V$ as above. Since (7.8.1) has a solution, we are in the situation $(6.7)(\mathrm{B})$. Thus $U$ is isomorphic to

$$
V^{\prime}=\left\{\begin{array}{c}
u^{2}+x^{3}+3 \nu x^{2} z^{2}+y^{3}+x y z^{3} \alpha^{\prime}\left(z^{3}\right) \\
+x z^{7} \beta^{\prime}\left(z^{3}\right)+y z^{8} \gamma^{\prime}\left(z^{3}\right)+z^{12} \delta^{\prime}\left(z^{3}\right)=0
\end{array}\right\} / \mathbb{Z}_{3}(2,1,1,0)
$$

for some $\nu \in \mathbb{C}$ and $\alpha^{\prime}(t), \beta^{\prime}(t), \gamma^{\prime}(t), \delta^{\prime}(t) \in \mathbb{C}\{t\}$. We have $\left(U,-\frac{1}{2} E_{\mid U}\right) \simeq$ $\left(V^{\prime},-\frac{1}{2} \operatorname{div}_{V^{\prime}}\left(y+A^{\prime}(x, y, z)\right)\right)$, where $A^{\prime}(x, y, z) \in(x, y, z)^{2} \mathbb{C}\{x, y, z\}$ is a 
$\mathbb{Z}_{3}$-semi-invariant. By $(2.12)$, there is a $K_{X_{1}}+f_{1 *}^{-1} \mathcal{H}_{X^{-}}$-repant Gorenstein resolution $g_{1}: Y \rightarrow X_{1}$ with $\# \mathcal{E}\left(g_{1}\right)=6$. Using (6.9), we know the value $a(F, X)$ for each $F \in \mathcal{E}\left(g_{1}\right)$. Thus $f=f_{1} \circ g_{1}: Y \rightarrow X$ is a required $K_{X}+\mathcal{H}_{X}$-crepant Gorenstein resolution.

(3) In case (C), let $f_{2}: X_{2} \rightarrow X, E_{2}, Q_{1} \in U_{1} \subseteq X_{2}, Q_{2} \in U_{2} \subseteq X_{2}$, $Q_{3} \in U_{3} \subseteq X_{2}$ be as in (7.8)(C), and let $g_{2}: Y \rightarrow X_{2}$ be the economic resolution of $Q_{1}$ and $Q_{2}$. Then $\mathcal{E}\left(g_{2}\right)=\left\{F_{1}, F_{2}, F_{3}, G_{1}, G_{2}\right\}$, where $F_{i}$ (resp. $\left.G_{j}\right)$ is the prime divisor over $Q_{1}$ (resp. $\left.Q_{2}\right)$ with $a\left(F_{i}, X_{2}\right)=i / 4(i=1,2$, 3) (resp. $\left.a\left(G_{j}, X_{2}\right)=j / 3(j=1,2)\right)$. By using (2.10) and (2.12), we see that $a\left(F_{1}, X\right)=a\left(F_{1}, X_{2},-\frac{1}{2} E_{2}\right)=a\left(F_{1}, U_{1},-\frac{1}{2} E_{2 \mid U_{1}}\right)=1 / 2$. Similarly we have $a\left(F_{2}, X\right)=1, a\left(F_{3}, X\right)=3 / 2$ and $a\left(G_{1}, X\right)=a\left(G_{2}, X\right)=1$. The birational transform $E=g_{2 *}^{-1} E_{2}$ satisfies $a(E, X)=1 / 2$.

(4) In case (D), let $f_{3}: X_{3} \rightarrow X, E_{3}, Q \in U \subseteq X_{3}$ be as in (7.8)(D), and let $g_{3}: Y \rightarrow X_{3}$ be the economic resolution of $Q$. Then $g_{3}$ is a $K_{X_{3}}+f_{3 *}^{-1} \mathcal{H}_{X^{-}}$ crepant Gorenstein resolution and we have $\mathcal{E}\left(g_{3}\right)=\left\{F_{1}, \ldots, F_{6}\right\}$, where $F_{i}$ is the prime divisor over $Q$ with $a\left(F_{i}, X_{3}\right)=i / 7(i=1, \ldots, 6)$. By $(2.10)$ and (2.12), we have $a\left(F_{1}, X\right)=a\left(F_{1}, X_{3},-\frac{1}{2} E_{3}\right)=a\left(F_{1}, U,-\frac{1}{2} E_{3 \mid U}\right)=1 / 2$. Similarly, we have $a\left(F_{2}, X\right)=1 / 2, a\left(F_{3}, X\right)=a\left(F_{4}, X\right)=a\left(F_{5}, X\right)=1$ and $a\left(F_{6}, X\right)=3 / 2$. Since $Y$ has only Gorenstein terminal singularities and since $a(E, X)=1 / 2$ for $E=g_{3 *}^{-1} E_{3}$, the composition $f=f_{3} \circ g_{3}: Y \rightarrow X$ is a $K_{X}+\mathcal{H}_{X}$-crepant Gorenstein resolution with required properties.

(5) In case (E), we construct birational morphisms $f_{3}: X_{3} \rightarrow X$ and $g_{3}: Y \rightarrow X_{3}$ as in (4). Then the composition $f=f_{3} \circ g_{3}: Y \rightarrow X$ is a $K_{X}+\mathcal{H}_{X}$-crepant Gorenstein resolution. Computations of discrepancies of each $F \in \mathcal{E}(f)$ is the same as in (4) except for $a\left(F_{1}, X\right)$. In this case, we have $a\left(F_{1}, X\right)=1$.

(6) Lastly we shall study case (F). Let $f_{4}: X_{4} \rightarrow X, E_{4}, Q_{1} \in U_{1} \subseteq$ $X_{4}, Q_{2} \in U_{2} \subseteq X_{4}$ be as in (7.8)(F), and let $g_{4}: Y \rightarrow X_{4}$ be the economic resolution of $Q_{1}$ and $Q_{2}$. Then $g_{4}$ is a $K_{X_{4}}+f_{4 *}^{-1} \mathcal{H}_{X^{-c r e p a n t}}$ Gorenstein resolution and we have $\mathcal{E}\left(g_{4}\right)=\left\{F_{1}, \ldots, F_{4}, G_{1}, G_{2}\right\}$, where $F_{i}$ (resp. $\left.G_{j}\right)$ is the prime divisor over $Q_{1}\left(\operatorname{resp} . Q_{2}\right)$ with $a\left(F_{i}, X_{4}\right)=i / 5$ $(i=1, \ldots, 4)\left(\operatorname{resp} . a\left(G_{j}, X_{4}\right)=j / 3(j=1,2)\right)$. By $(2.10)$ and $(2.12)$, we have $a\left(F_{1}, X\right)=a\left(F_{1}, X_{4},-\frac{1}{2} E_{4}\right)=a\left(F_{1}, U_{1},-\frac{1}{2} E_{4 \mid U_{1}}\right)=1 / 2$. Similarly, we have $a\left(F_{2}, X\right)=a\left(F_{3}, X\right)=a\left(F_{4}, X\right)=1, a\left(G_{1}, X\right)=1 / 2$ and $a\left(G_{2}, X\right)=1$. Since $Y$ has only Gorenstein terminal singularities and since $a(E, X)=1 / 2$ for $E=g_{4 *}^{-1} E_{4}$, the composition $f=f_{4} \circ g_{4}: Y \rightarrow X$ satisfies our requirement.

Remark 7.10. By (7.3), (7.4), (7.5), (7.7) and (7.9), our main results 
(3.1), (3.2) and (3.3) are proved in the case $X$ is of type (cE/2). Moreover, if $D_{X} \in \mathcal{H}_{X}$ has only rational double point of type $E_{7}$, then our Gorenstein resolution $f: Y \rightarrow X$ induces the minimal resolution $f_{\mid D_{Y}}: D_{Y}=$ $f_{*}^{-1} D_{X} \rightarrow D_{X}$ of $D_{X}$ except for $(7.9)(\mathrm{C})$. In this exceptional case, $D_{Y}$ has one rational double point of type $A_{1}$ and $Y$ has an isolated cDV point there.

\section{$\S 8$. Terminal singularities of type $(\mathrm{cD} / 2)$}

In this section, we shall study a part of 3-dimensional terminal singularities of type $(\mathrm{cD} / 2)$. The rest of this type will be treated in Section 9 together with type $(\mathrm{cAx} / 4)$.

8.1. Let $X$ be a germ of a 3-dimensional terminal singularity of type $(\mathrm{cD} / 2)$ and assume that there is an embedding $X \hookrightarrow(x, y, z, u) / \mathbb{Z}_{2}(1,1,0,1)$ such that

$$
X=\left\{u^{2}+x y z+x^{2 a}+y^{2 b}+z^{c}=0\right\} / \mathbb{Z}_{2}(1,1,0,1),
$$

where $a, b \geq 2$ and $c \geq 3$. Let $\mathcal{H}_{X} \subseteq\left|-K_{X}\right|$ be a linear system such that a general member has only a rational double point. We remark that the general member of $\left|-K_{X}\right|$ has a rational double point of type $D_{2 c}$.

We first treat the case $a=b=2$, and next treat the case $a \geq 3$ or $b \geq 3$.

The following lemma is a special case of (4.6) and will be used in the proof of (8.3).

LEMmA 8.2. Let $U=\left\{x y+z u^{2}+z^{c-2}=0\right\} / \mathbb{Z}_{2}(1,1,0,1)(c \geq 3)$, $\mathcal{H}_{U} \subseteq\left|-K_{U}\right|$ be a linear system such that a general member has only a rational double point and let $\Delta=-\frac{1}{2} \operatorname{div}_{U}(z)$. Then there is a $K_{U}+\mathcal{H}_{U}$ crepant Gorenstein resolution $g: V \rightarrow U$ which satisfies the following:

(1) If $c=3$, then $\# \mathcal{E}(g)=1$ with $\# \mathcal{E}(g, \Delta, 1)=1$.

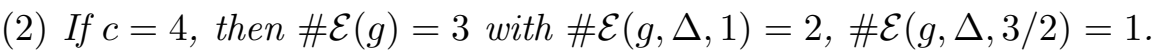

(3) If $c \geq 5$, then $\# \mathcal{E}(g)=c-1$ with $\# \mathcal{E}(g, \Delta, 1)=\# \mathcal{E}(g, \Delta, 3 / 2)=2$ and $\# \mathcal{E}(g, \Delta, i / 2)=1$ for each $i=4,5, \ldots, c-2$.

Proof. Since the weight sequence of $U$ as type (cA/2) (cf. (4.3)) is (1) if $c=3$, and $(2,3, \ldots, c-2)$ if $c \geq 4$, this lemma follows from (4.6). 
THEOREM 8.3. Let $X, \mathcal{H}_{X}$ be as in (8.1) and assume that $a=b=2$. Then there is a $K_{X}+\mathcal{H}_{X}$-crepant Gorenstein resolution $f: Y \rightarrow X$ which satisfies the following:

(1) If $c=3$, then we have $\# \mathcal{E}(f)=4$ with $\# \mathcal{E}(f, 1 / 2)=1, \# \mathcal{E}(f, 1)=2$ and $\# \mathcal{E}(f, 3 / 2)=1$.

(2) If $c=4$, then we have $\# \mathcal{E}(f)=6$ with $\# \mathcal{E}(f, 1 / 2)=1, \# \mathcal{E}(f, 1)=3$ and $\# \mathcal{E}(f, 3 / 2)=2$.

(3) If $c \geq 5$, then we have $\# \mathcal{E}(f)=c+2$ with $\# \mathcal{E}(f, 1 / 2)=1$, $\# \mathcal{E}(f, 1)=\# \mathcal{E}(f, 3 / 2)=3$ and $\# \mathcal{E}(f, i / 2)=1$ for each $i=4,5, \ldots, c-2$.

Proof. By using [Hay00, 4.4], the blow up $f_{1}: X_{1} \rightarrow X$ with weight $(x, y, z, u)=\frac{1}{2}(1,1,2,3)$ is divisorial with discrepancy $1 / 2$, and $X_{1}$ is Gorenstein outside two points $Q_{1}$ and $Q_{2}$. One point $Q_{1}$ has an open neighborhood $U_{1} \subseteq X_{1}$ such that $U_{1} \simeq\left\{x y+z u^{2}+z^{c-2}=0\right\} / \mathbb{Z}_{2}(1,1,0,1)$, and the other point $Q_{2}$ has an open neighborhood $U_{2} \subseteq X_{1}$ such that $U_{2} \simeq(x, y, z) / \mathbb{Z}_{3}(1,1,2)$. We also see that $E_{1 \mid U_{1}}=\operatorname{div}_{U_{1}}(z)$ and $E_{1 \mid U_{2}}=$ $\operatorname{div}_{U_{2}}\left(x y z+x^{4}+y^{4}\right)$ for the exceptional divisor $E_{1}$ of $f_{1}$.

By using (8.2) for $Q_{1}$ and the economic resolution of $Q_{2}$, we get a $K_{X_{1}}+f_{1 *}^{-1} \mathcal{H}_{X}$-crepant Gorenstein resolution $g_{1}: Y \rightarrow X_{1}$ such that $\mathcal{E}\left(g_{1}\right)=$ \{prime divisors over $\left.Q_{1}\right\} \cup\left\{G_{1}, G_{2}\right\}$, where $G_{j}$ is the prime divisor over $Q_{2}$ with $a\left(G_{j}, X_{1}\right)=j / 3(j=1,2)$. By $(2.10)$ and $(2.12)$, we have $a\left(G_{1}, X\right)=$ $a\left(G_{1}, X_{1},-\frac{1}{2} E_{1}\right)=a\left(G_{1}, U_{2},-\frac{1}{2} E_{1 \mid U_{2}}\right)=1$, and similarly $a\left(G_{2}, X\right)=3 / 2$. We know that $a(F, X)=a\left(F, X_{1},-\frac{1}{2} E_{1}\right)=a\left(F, U_{1},-\frac{1}{2} \operatorname{div}_{U_{1}}(z)\right)$ for a prime divisor $F$ over $Q_{1}$ by (2.10), and these values are obtained in (8.2). Since $a(E, X)=1 / 2$ for the birational transform $E=g_{1 *}^{-1} E_{1}$, we complete the proof.

Next we shall treat the case $a \geq 3$ or $b \geq 3$. By changing $x$ and $y$ if necessary, we shall assume that $a \geq 3$. The following lemmas will be used in the proof of (8.6) and (8.7).

LEMma 8.4. Let $U=\left\{x z+u^{2}+y^{3 b-3}=0\right\} / \mathbb{Z}_{3}(1,1,2,0)(b \geq 2)$, $\mathcal{H}_{U} \subseteq\left|-K_{U}\right|$ be a linear system such that a general member has only a rational double point and let $\Delta=-\frac{1}{2} \operatorname{div}_{U}(y)$. Then there is a $K_{U}+\mathcal{H}_{U^{-}}$ crepant Gorenstein resolution $g: V \rightarrow U$ which satisfies the following:

(1) If $b=2$, then $\# \mathcal{E}(g)=4$ with $\# \mathcal{E}(g, \Delta, 1 / 2)=1, \# \mathcal{E}(g, \Delta, 1)=2$ and $\# \mathcal{E}(g, \Delta, 3 / 2)=1$.

(2) If $b \geq 3$, then $\# \mathcal{E}(g)=5$ with $\# \mathcal{E}(g, \Delta, 1 / 2)=\# \mathcal{E}(g, \Delta, 1)=2$ and $\# \mathcal{E}(g, \Delta, 3 / 2)=1$. 
Proof. (1) By [Hay99, 6.4], the blow up $g_{1}: U_{1} \rightarrow U$ with weight $(x, y, z, u)=\frac{1}{3}(1,1,2,3)$ is divisorial with discrepancy $1 / 3$, and $U_{1}$ is Gorenstein outside two points $Q_{1}$ and $Q_{2}$. One point $Q_{1}$ has an open neighborhood $W_{1} \subseteq U_{1}$ such that $W_{1} \simeq(y, z, u) / \mathbb{Z}_{2}(1,1,1)$, and the other point $Q_{2}$ has an open neighborhood $W_{2} \subseteq U_{1}$ such that $W_{2} \simeq(x, y, z) / \mathbb{Z}_{3}(1,1,2)$. For the exceptional divisor $E_{1}$ of $g_{1}$, we have $\left(g_{1}^{*} \Delta-\frac{1}{3} E_{1}\right)_{\mid W_{1}}=-\frac{1}{2} \operatorname{div}_{W_{1}}(y)-$ $\frac{1}{2} \operatorname{div}_{W_{1}}(z)$ and $\left(g_{1}^{*} \Delta-\frac{1}{3} E_{1}\right)_{\mid W_{2}}=-\frac{1}{2} \operatorname{div}_{W_{2}}(y)-\frac{1}{2} \operatorname{div}_{W_{2}}\left(x z+y^{3}\right)$.

Let $h_{1}: V \rightarrow U_{1}$ be the economic resolution of $Q_{1}$ and $Q_{2}$. Then $V$ has only Gorenstein terminal singularities and $\mathcal{E}\left(h_{1}\right)=\left\{F, G_{1}, G_{2}\right\}$, where $F$ (resp. $G_{j}$ ) is the prime divisor over $Q_{1}$ (resp. $Q_{2}$ ) with $a\left(F, U_{1}\right)=1 / 2$ (resp. $\left.a\left(G_{i}, U_{1}\right)=j / 3(j=1,2)\right)$. By $(2.10)$ and $(2.12)$, we have $a(F, U, \Delta)=$ $a\left(F, U_{1}, g_{1}^{*} \Delta-\frac{1}{3} E_{1}\right)=1$ and similarly $a\left(G_{1}, U, \Delta\right)=1, a\left(G_{2}, U, \Delta\right)=3 / 2$. Hence the composition $g=g_{1} \circ h_{1}: V \rightarrow U$ satisfies our requirement.

(2) In this case, we start with the blow up $g_{2}: U_{2} \rightarrow U$ with weight $(x, y, z, u)=\frac{1}{3}(1,1,5,3)$, which is divisorial with discrepancy $1 / 3$. We see that $U_{2}$ has a unique non-Gorenstein point, which is a cyclic quotient terminal singularity of index 5 . Thus we can argue as in (1) and get the results. We shall omit the details.

Lemma 8.5. Let $U=\left\{x y+u^{2}+z^{c-3}=0\right\} / \mathbb{Z}_{2}(1,1,0,1)(c \geq 4)$, $\mathcal{H}_{U} \subseteq\left|-K_{U}\right|$ be a linear system such that a general member has only a rational double point and let $\Delta=-\frac{1}{2} \operatorname{div}_{U}(z)$. Then there is a $K_{U}+\mathcal{H}_{U^{-}}$ crepant Gorenstein resolution $g: V \rightarrow U$ such that $\# \mathcal{E}(g)=c-3$ with $\# \mathcal{E}(g, \Delta, i / 2)=1$ for each $i=2,3, \ldots, c-2$.

Proof. Since the weight sequence of $U$ as type (cA/2) (cf. (4.3)) is $(1,2, \ldots, c-3)$, this lemma follows from (4.6).

Theorem 8.6. Let $X, \mathcal{H}_{X}$ be as in (8.1) and assume that $a \geq 3$ and $b=2$. Then there is a $K_{X}+\mathcal{H}_{X}$-crepant Gorenstein resolution $f: Y \rightarrow X$ which satisfies the following:

(1) If $c=3$, then we have $\# \mathcal{E}(f)=5$ with $\# \mathcal{E}(f, 1 / 2)=\# \mathcal{E}(f, 1)=2$ and $\# \mathcal{E}(f, 3 / 2)=1$.

(2) If $c=4$, then we have $\# \mathcal{E}(f)=6$ with $\# \mathcal{E}(f, 1 / 2)=2, \# \mathcal{E}(f, 1)=3$ and $\# \mathcal{E}(f, 3 / 2)=1$.

(3) If $c \geq 5$, then we have $\# \mathcal{E}(f)=c+2$ with $\# \mathcal{E}(f, 1 / 2)=2$, $\# \mathcal{E}(f, 1)=3$ and $\# \mathcal{E}(f, 3 / 2)=2$ and $\# \mathcal{E}(f, i / 2)=1$ for each $i=4$, $5, \ldots, c-2$. 
TheOREM 8.7. Let $X, \mathcal{H}_{X}$ be as in (8.1) and assume that $a, b \geq 3$. Then there is a $K_{X}+\mathcal{H}_{X}$-crepant Gorenstein resolution $f: Y \rightarrow X$ which satisfies the following:

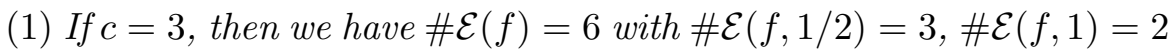
and $\# \mathcal{E}(f, 3 / 2)=1$.

(2) If $c=4$, then we have $\# \mathcal{E}(f)=7$ with $\# \mathcal{E}(f, 1 / 2)=\# \mathcal{E}(f, 1)=3$ and $\# \mathcal{E}(f, 3 / 2)=1$.

(3) If $c \geq 5$, then we have $\# \mathcal{E}(f)=c+3$ with $\# \mathcal{E}(f, 1 / 2)=\# \mathcal{E}(f, 1)=$ $3, \# \mathcal{E}(f, 3 / 2)=2$ and $\# \mathcal{E}(f, i / 2)=1$ for each $i=4,5, \ldots, c-2$.

We shall prove these two theorems at the same time.

Proof. We first assume that $c \geq 4$. By [Hay00, 4.7, 4.12], the blow up $f_{1}: X_{1} \rightarrow X$ with weight $(x, y, z, u)=\frac{1}{2}(1,3,2,3)$ is divisorial with discrepancy $1 / 2$, and $X_{1}$ is Gorenstein outside two points $Q_{1}$ and $Q_{2}$. One point $Q_{1}$ has an open neighborhood $U_{1} \subseteq X_{1}$ such that $U_{1} \simeq\left\{x z+u^{2}+\right.$ $\left.y^{3 b-3}=0\right\} / \mathbb{Z}_{3}(1,1,2,0)$, and the other point $Q_{2}$ has an open neighborhood $U_{2} \subseteq X_{1}$ such that $U_{2} \simeq\left\{x y+u^{2}+z^{c-3}=0\right\} / \mathbb{Z}_{2}(1,1,0,1)$. For the exceptional divisor $E_{1}$ of $f_{1}$, we have $E_{1 \mid U_{1}}=\operatorname{div}_{U_{1}}(y)$ and $E_{1 \mid U_{2}}=\operatorname{div}_{U_{2}}(z)$.

By taking the $K_{X_{1}}+f_{1 *}^{-1} \mathcal{H}_{X}$-crepant Gorenstein resolutions of $Q_{1}$ and $Q_{2}$ as in (8.4) and (8.5), we get a projective birational morphism $g_{1}: Y \rightarrow X_{1}$ such that $Y$ has only Gorenstein terminal singularities. If $F_{1}$ is a prime divisor over $Q_{1}$, we have $a\left(F_{1}, X\right)=a\left(F_{1}, X_{1},-\frac{1}{2} E_{1}\right)=$ $a\left(F_{1}, U_{1},-\frac{1}{2} \operatorname{div}_{U_{1}}(y)\right)$. We also see that if $F_{2}$ is a prime divisor over $Q_{2}$, then $a\left(F_{2}, X\right)=a\left(F_{2}, U_{2},-\frac{1}{2} \operatorname{div}_{U_{2}}(z)\right)$. Thus we know the value $a(F, X)$ for each $F \in \mathcal{E}\left(g_{1}\right)$ by (8.4) and (8.5). Since the birational transform $E=g_{1 *}^{-1} E_{1}$ satisfies $a(E, X)=1 / 2$, the composition $f=f_{1} \circ g_{1}: Y \rightarrow X$ is a $K_{X}+\mathcal{H}_{X}$-crepant Gorenstein resolution of $X$ and $\mathcal{E}(f)$ satisfies the conditions in (8.6) and (8.7). Thus we proved (8.6) and (8.7) when $c \geq 4$.

If $c=3$, then the same blow up $f_{1}: X_{1} \rightarrow X$ as above is divisorial with discrepancy $1 / 2$, and $X_{1}$ is Gorenstein outside one point $Q_{1} \in X_{1}$, which has the same properties as above. Thus we can argue similarly and obtain $(8.6)(1)$ and $(8.7)(1)$

Remark 8.8. By (8.3), (8.6) and (8.3), we obtain our main results (3.1), (3.2) and (3.3) for $X$ as in (8.1), which is a part of type $(\mathrm{cD} / 2)$. In this case $f_{\mid D_{Y}}: D_{Y}=f_{*}^{-1} D_{X} \rightarrow D_{X}$ is not necessarily the minimal resolution for $D_{X} \in \mathcal{H}_{X}$. 


\section{§9. Terminal singularities of type $(\mathrm{cD} / 2)$ and $(\mathrm{cAx} / 4)$}

In this section, we shall study 3-dimensional terminal singularities of type $(\mathrm{cD} / 2)$ and $(\mathrm{cAx} / 4)$ simultaneously. The argument in this section is similar to the one given in Section 4, but rather complicated. The results in this section and Section 8 treat all 3-dimensional terminal singularities of type $(\mathrm{cD} / 2)$. All type $(\mathrm{cAx} / 4)$ singularities are treated in this section.

9.1. Let $X$ be a germ of a 3 -dimensional terminal singularity at $P$. We assume that there is an embedding $X \hookrightarrow(x, y, z, u) / \mathbb{Z}_{2}(1,1,1,0)$ such that

$$
X=\left\{x^{2}+y^{2} u+h(z) y+g(z, u)=0\right\} / \mathbb{Z}_{2}(1,1,1,0),
$$

where $h(z)=\sum_{r \geq 0} b_{r} z^{2 r+1} \in \mathbb{C}\{z\}$ and $g(z, u)=\sum_{p+q \geq 1} a_{p q} z^{2 p} u^{q} \in$ $\mathbb{C}\left\{z^{2}, u\right\}$.

For each positive integer $i$, we denote $\tau_{i}$-wt $(z, u)=(i / 2,2)$, and define

$$
\begin{aligned}
w_{i}(X) & =\tau_{i}-w t\left(-\frac{1}{4} h(z)^{2}+g(z, u) u\right)-2 \\
& =\min \left\{2 \tau_{i}-w t(h(z))-2, \tau_{i}-w t(g(z, u))\right\} \\
& =\min \left(\left\{(2 r+1) i-2 \mid b_{r} \neq 0\right\} \cup\left\{i p+2 q \mid a_{p q} \neq 0\right\}\right) .
\end{aligned}
$$

Since the cyclic group $\mathbb{Z}_{2}$ acts freely on $\left\{x^{2}+y^{2} u+h(z) y+g(z, u)=\right.$ $0\} \backslash\{(0)\}$, we see that $u^{w} \in g(z, u)$ for some positive integer $w$. The smallest such integer $w$ is called the axial weight of $X$ and we shall denote it by $\operatorname{aw}(X)$ (cf. [Hay99, 2.6]).

Let $\mathcal{H}_{X} \subseteq\left|-K_{X}\right|$ be a linear system such that a general member has only a rational double point. We remark that $X$ is of type $(\mathrm{cD} / 2)$ in most cases, but it may be of type $(\mathrm{cAx} / 2)$ or $(\mathrm{cA} / 2)$. If $X$ is of type $(\mathrm{cD} / 2)$, then a general member of $\left|-K_{X}\right|$ has a rational double point of type $D_{2 a w}(X)$.

LEMMA 9.2. Under the notation and assumptions in (9.1), we have the following:

(1) For each positive integer $i$, we have $-1 \leq w_{i}(X) \in \mathbb{Z}, w_{i}(X) \leq$ $w_{i+1}(X)$ and $w_{i+1}(X)-w_{i}(X) \geq w_{i+2}(X)-w_{i+1}(X)$.

(2) If $i$ is even, then $w_{i}(X)$ is also even.

(3) For all sufficiently large integer $i$, we have $w_{i}(X)=2 \mathrm{aw}(X)$.

(4) If $w_{1}(X) \leq 2$, then $\tau_{1}-w t(h(z)) \leq 1$ or $\tau_{1}$-wt $(g(z, u)) \leq 2$. Furthermore,

$$
\begin{aligned}
\tau_{1}-w t(h(z)) \leq 1 & \text { if and only if } b_{0} \neq 0 \text { or } b_{1} \neq 0 \\
\tau_{1}-w t(g(z, u)) \leq 2 & \text { if and only if } a_{10} \neq 0, a_{20} \neq 0 \text { or } a_{01} \neq 0 .
\end{aligned}
$$


Proof. We can prove (1) and (3) similarly as in (4.2), while proofs for (2) and (4) are obvious.

9.3. By (9.2), we get a sequence of strictly increasing integers

$$
\left(w_{1}(X), w_{2}(X), \ldots, w_{l}(X)\right),
$$

where $w_{l}(X)=2 \mathrm{aw}(X)$. This is called the weight sequence of $X$ as type $(\mathrm{cD} / 2)$. We also call $l=l(X)$ the length of the weight sequence of $X$.

For each positive odd integer $i$, we define

$$
\bar{w}_{i}(X)= \begin{cases}w_{i}(X) & \text { if } w_{i}(X) \text { is odd } \\ w_{i}(X)-1 & \text { if } w_{i}(X) \text { is even }\end{cases}
$$

In particular, $\bar{w}_{i}(X)$ is always an odd integer. We sometimes denote $w_{i}(X)$ and $\bar{w}_{i}(X)$ by $w_{i}$ and $\bar{w}_{i}$ respectively if there is no danger of confusion.

For each positive even integer $i$, we shall denote $G_{i}(z, u)=\left(-\frac{1}{4} h(z)^{2}+\right.$ $g(z, u) u)_{\tau_{i-w t}=w_{i}(X)+2}$ and set $\chi_{i}(z)=G_{i}(z, 1)$. We define the condition $(* i)$ as follows:

$$
\chi_{i}(z)=\left(\sum_{t \geq 1} \alpha_{t} z^{2 t-1}\right)^{2} \text { for some } \alpha_{t} \in \mathbb{C} .
$$

If $i \notin 4 \mathbb{Z}$, then the condition $(* i)$ is equivalent to $\left(* i^{\prime}\right) \quad \begin{cases}w_{i}(X) \notin 4 \mathbb{Z}, & (g(z, u))_{\tau_{i}-w t=w_{i}(X)} \text { is a square, or } \\ w_{i}(X) \in 4 \mathbb{Z}, & \left(-\frac{1}{4} h(z)^{2}+g(z, u) u\right)_{\tau_{i}-w t=w_{i}(X)+2} \text { is a square. }\end{cases}$

It is easy to see that the condition $(* i)$ does not hold for all $i \geq l(X)$.

In the following, we shall consider the pair $\left(X, \Delta_{X}(k)\right)$, where $X$ is as in (9.1) and $\Delta_{X}(k)=-\frac{k}{4} \operatorname{div}_{X}(u)$ with $k \in \mathbb{Q}$. This allows us to work inductively.

9.4. Let $\hat{X}$ be a germ of a 3 -dimensional terminal singularity at $\hat{P} \in$ $\hat{X}$. We assume that there is an embedding $\hat{X} \hookrightarrow(x, y, z, u) / \mathbb{Z}_{4}(1,3,1,2)$ such that

$$
\hat{X}=\left\{x^{2}+y^{2}+f(z, u)=0\right\} / \mathbb{Z}_{4}(1,3,1,2),
$$

where $f(z, u)=\sum_{p+q \geq 1, o d d} c_{p q} z^{2 p} u^{q} \in \mathbb{C}\left\{z^{2}, u\right\}$.

For each positive integer $i$, we denote $\rho_{i}$-wt $(z, u)=(i / 2,1)$, and define

$$
\hat{w}_{i}(\hat{X})=\rho_{i}-w t(f(z, u))=\min \left\{i p+q \mid c_{p q} \neq 0\right\} .
$$


Since the cyclic group $\mathbb{Z}_{4}$ acts freely on $\left\{x^{2}+y^{2}+f(z, u)=0\right\} \backslash\{(0)\}$, we see that $u^{w} \in f(z, u)$ for some positive odd integer $w$. Let $w$ be the smallest such integer, then we have $\operatorname{aw}(\hat{X})=(w+1) / 2($ cf. [Hay99, 2.6]).

Let $\mathcal{H}_{\hat{X}} \subseteq\left|-K_{\hat{X}}\right|$ be a linear system such that a general member has only a rational double point. We remark that $\hat{X}$ is of type $(\mathrm{cAx} / 4)$ if $c_{01} \neq 0$. If this is so, then a general member of $\left|-K_{\hat{X}}\right|$ has a rational double point of type $D_{2 \mathrm{aw}(\hat{X})+1}$

LEMMA 9.5. Under the notation and assumptions in (9.4), we have the following:

(1) For each positive integer $i$, we have $1 \leq \hat{w}_{i}(\hat{X}) \in \mathbb{Z}, \hat{w}_{i}(\hat{X}) \leq$ $\hat{w}_{i+1}(\hat{X})$ and $\hat{w}_{i+1}(\hat{X})-\hat{w}_{i}(\hat{X}) \geq \hat{w}_{i+2}(\hat{X})-\hat{w}_{i+1}(\hat{X})$.

(2) If $i$ is odd, then $\hat{w}_{i}(\hat{X})$ is also odd.

(3) For all sufficiently large integer $i$, we have $\hat{w}_{i}(\hat{X})=2 \operatorname{aw}(\hat{X})-1$.

Proof. This lemma is proved similarly as in (4.2) or (9.2).

9.6. By (9.5), we get a sequence of strictly increasing positive integers

$$
\left(\hat{w}_{1}(\hat{X}), \hat{w}_{2}(\hat{X}), \ldots, \hat{w}_{l}(\hat{X})\right)
$$

where $\hat{w}_{l}(\hat{X})=2 \operatorname{aw}(\hat{X})-1$. This is called the weight sequence of $\hat{X}$ as type $(\mathrm{cAx} / 4)$. We also call $l=\hat{l}(\hat{X})$ the length of the weight sequence of $\hat{X}$. We sometimes denote $\hat{w}_{i}(\hat{X})$ by $\hat{w}_{i}$ if there is no danger of confusion.

For each positive odd integer $i$, we define the condition $(\dagger i)$ as follows:

$$
(f(z, u))_{\rho_{i}-w t=\hat{w}_{i}(\hat{X})}=\sum_{i p+q=\hat{w}_{i}(\hat{X})} c_{p q} z^{2 p} u^{q} \text { is a square. }
$$

If we set $F_{i}(z, u)=(f(z, u))_{\rho_{i}-w t=\hat{w}_{i}(\hat{X})}$ and $\hat{\chi}_{i}(z)=F_{i}(z, 1)$, then the condition $(\dagger i)$ is equivalent to

$$
\hat{\chi}_{i}(z)=\left(\sum_{t \geq 1} \beta_{t} z^{2 t-1}\right)^{2} \text { for some } \beta_{t} \in \mathbb{C}
$$

since $\hat{w}_{i}(\hat{X})$ is odd. It is easy to see that the condition $(\dagger i)$ does not hold for all $i \geq \hat{l}(\hat{X})$

We shall consider the pair $\left(\hat{X}, \hat{\Delta}_{\hat{X}}(k)\right)$, where $\hat{X}$ is as in (9.4) and $\hat{\Delta}_{\hat{X}}(k)=-\frac{k}{2} \operatorname{div}_{\hat{X}}(u)$ with $k \in \mathbb{Q}$. This again allows us to work inductively together with $\left(X, \Delta_{X}(k)\right)$ in $(9.3)$. 
From now on, we shall study Gorenstein resolutions of $X$ in (9.1) and $\hat{X}$ in (9.4) by dividing into several cases. The results in this section will be given in (9.19) and (9.20) after we prepare several inductive steps in (9.7)-(9.18).

The first two lemmas (9.7) and (9.8) deals with $X$ as in (9.1) such that $X$ is not of type $(\mathrm{cD} / 2)$. We need these lemmas because of the inductive argument in (9.19) and (9.20).

Lemma 9.7. Let $X, w_{i}(X), \Delta_{X}(k), \mathcal{H}_{X}$ be as in (9.1) and (9.3), and assume that $\bar{w}_{1}(X)=-1$. Then $w_{2}(X)=0$, the condition $(* 2)$ holds, and there is a $K_{X}+\mathcal{H}_{X}$-crepant Gorenstein resolution $f: Y \rightarrow X$ such that $\# \mathcal{E}(f)=\operatorname{aw}(X)$ with

$$
\# \mathcal{E}\left(f, \Delta_{X}(k),(k+2 i) / 4\right)=1 \text { for } i=1,2, \ldots, \text { aw }(X) .
$$

Proof. Since $z \in h(z)$, we see that $w_{2}(X)=0$, the condition $(* 2)$ holds and that

$$
\left(X, \Delta_{X}(k)\right) \simeq\left(\left\{x^{2}+y z+u^{\mathrm{aw}(X)}=0\right\} / \mathbb{Z}_{2}(1,1,1,0),-\frac{k}{4} \operatorname{div}(u)\right) .
$$

Thus we immediately see that the weight sequence of $X$ as type $(\mathrm{cA} / 2)$ (cf. $(4.3))$ is $(1,2, \ldots$, aw $(X))$. Therefore the remaining part of this lemma follows from (4.6).

Lemma 9.8. Let $X, w_{i}(X), \Delta_{X}(k), \mathcal{H}_{X}$ be as in (9.1) and (9.3), and assume that $\bar{w}_{1}(X)=1$ and $w_{2}(X)=2$. Then the condition $(* 2)$ holds if and only if $\operatorname{aw}(X) \geq 2$, and one of the following holds:

(1) If the condition $(* 2)$ holds, then there is a $K_{X}+\mathcal{H}_{X}$-crepant Gorenstein resolution $f: Y \rightarrow X$ such that $\# \mathcal{E}(f)=\operatorname{aw}(X)+1$. Moreover, if $\operatorname{aw}(X)=2$, then

$$
\# \mathcal{E}\left(f, \Delta_{X}(k),(k+2 i) / 4\right)= \begin{cases}2 & \text { if } i=1, \\ 1 & \text { if } i=2,\end{cases}
$$

and if $\operatorname{aw}(X) \geq 3$, then

$$
\# \mathcal{E}\left(f, \Delta_{X}(k),(k+2 i) / 4\right)= \begin{cases}2 & \text { if } i=1,2, \\ 1 & \text { if } i=3, \ldots, \text { aw }(X)-1 .\end{cases}
$$

(2) If the condition $(* 2)$ does not hold, then there is a $K_{X}+\mathcal{H}_{X}$-crepant Gorenstein resolution $f: Y \rightarrow X$ such that $\# \mathcal{E}(f)=1$ with

$$
\# \mathcal{E}\left(f, \Delta_{X}(k),(k+2) / 4\right)=1 \text {. }
$$


Proof. Since $a_{10} \neq 0$ or $a_{01} \neq 0$, the condition $(* 2)$ holds if and only if $a_{01}=0$, which is equivalent to saying that $\operatorname{aw}(X) \geq 2$.

If the condition $(* 2)$ holds, then we have

$$
\left(X, \Delta_{X}(k)\right) \simeq\left(\left\{x^{2}+z^{2}+y^{2} u+u^{\mathrm{aw}(X)}=0\right\} / \mathbb{Z}_{2}(1,1,1,0),-\frac{k}{4} \operatorname{div}(u)\right)
$$

Thus the weight sequence of $X$ as type (cA/2) (cf. (4.3)) is $(2,3, \ldots$, aw $(X))$. Hence this lemma follows from (4.6).

If the condition $(* 2)$ does not hold, then $X \simeq(x, y, z) / \mathbb{Z}_{2}(1,1,1)$. Let $f: Y \rightarrow X$ be the economic resolution, then the exceptional divisor $E$ of $f$ satisfies $a\left(E, X, \Delta_{X}(k)\right)=(k+2) / 4$.

Lemma 9.9. Let $X, w_{i}(X), \Delta_{X}(k), \mathcal{H}_{X}$ be as in (9.1) and (9.3), and assume that $\bar{w}_{1}(X)=1, w_{2}(X)=4$ and that the condition $(* 2)$ does not hold. Then one of the following holds:

(1) If $\operatorname{aw}(X)=2$, then there is a $K_{X}+\mathcal{H}_{X}$-crepant Gorenstein resolution $f_{1}: X_{1} \rightarrow X$ such that $\# \mathcal{E}\left(f_{1}\right)=3$ with

$$
\begin{aligned}
& \# \mathcal{E}\left(f_{1}, \Delta_{X}(k),(k+2) / 4\right)=1, \quad \# \mathcal{E}\left(f_{1}, \Delta_{X}(k),(k+4) / 4\right)=1, \\
& \# \mathcal{E}\left(f_{1}, \Delta_{X}(k),(k+2) / 2\right)=1
\end{aligned}
$$

(2) If aw $(X) \geq 3$, then there is a $K_{X}+\mathcal{H}_{X}$-crepant birational morphism $f_{1}: X_{1} \rightarrow X$ such that $\# \mathcal{E}\left(f_{1}\right)=3$ with

$$
\begin{aligned}
& \# \mathcal{E}\left(f_{1}, \Delta_{X}(k),(k+2) / 4\right)=1, \quad \# \mathcal{E}\left(f_{1}, \Delta_{X}(k),(k+2) / 2\right)=1 \\
& \# \mathcal{E}\left(f_{1}, \Delta_{X}(k),(k+3) / 2\right)=1
\end{aligned}
$$

and that $X_{1}$ is Gorenstein terminal outside one point $P_{1}$. There is an open neighborhood $P_{1} \in U \subseteq X_{1}$ such that

$$
U \simeq\left\{x^{2}+y^{2} u-\frac{1}{4} h\left(z u^{1 / 2}\right)^{2} / u^{3}+g\left(z u^{1 / 2}, u\right) / u^{2}=0\right\} / \mathbb{Z}_{2}(1,1,1,0) .
$$

Furthermore, we have $a\left(G, X, \Delta_{X}(k)\right)=a\left(G, U,-\frac{k+2}{4} \operatorname{div}_{U}(u)\right)$ for each prime divisor $G$ over $P_{1}$.

Proof. Let $g_{1}: Y_{1} \rightarrow X$ be the blow up with weight $(x, y, z, u)=$ $\frac{1}{2}(3,1,1,2)$. By [Hay00, 5.22, 5.32], $g_{1}$ is divisorial with discrepancy $1 / 2$ and $Y_{1}$ is Gorenstein outside the origin $Q_{1}$ of the $x$-chart and the origin $Q_{4}$ of the $u$-chart. Let $E$ be the exceptional divisor of $g_{1}$. 
We shall study these non-Gorenstein points individually. There is an open neighborhood $Q_{1} \in U_{1} \subseteq Y_{1}$ such that $U_{1} \simeq(y, z, u) / \mathbb{Z}_{3}(1,1,2)$. We see that $a\left(E, X, \Delta_{X}(k)\right)=(k+2) / 4$ and that

$$
\begin{aligned}
& \left(g_{1}^{*} \Delta_{X}(k)-\frac{1}{2} E\right)_{\mid U_{1}} \\
& \quad=-\frac{k}{4} \operatorname{div}_{U_{1}}(u)-\frac{k+2}{4} \operatorname{div}_{U_{1}}\left(y^{2} u+b_{1} z^{3} y+a_{20} z^{4}+a_{11} z^{2} u+a_{02} u^{2}\right) .
\end{aligned}
$$

We take the economic resolution $h_{1}: X_{1} \rightarrow Y_{1}$ of $Q_{1}$, then $\mathcal{E}\left(h_{1}\right)=\left\{F_{1}, F_{2}\right\}$, where $F_{j}$ is the prime divisor over $Q_{1}$ with $a\left(F_{j}, Y_{1}\right)=j / 3(j=1,2)$. We see that

$$
\begin{aligned}
a\left(F_{1}, X, \Delta_{X}(k)\right) & =(k+2) / 2 \\
\text { and } \quad a\left(F_{2}, X, \Delta_{X}(k)\right) & = \begin{cases}(k+3) / 2 & \text { if } a_{02}=0, \\
(k+4) / 4 & \text { if } a_{02} \neq 0\end{cases}
\end{aligned}
$$

by using (2.10) and (2.12). We can write the $u$-chart $U_{4}$ as follows:

$$
U_{4}=\left\{x^{2} u+y^{2}+h\left(z u^{1 / 2}\right) / u^{3 / 2} \cdot y+g\left(z u^{1 / 2}, u\right) / u^{2}=0\right\} / \mathbb{Z}_{2}(1,1,1,0),
$$

which is isomorphic to the given form of $U$. The composition $f_{1}=g_{1} \circ$ $h_{1}: X_{1} \rightarrow X$ is a projective birational morphism such that $\mathcal{E}\left(f_{1}\right)=$ $\left\{h_{1 *}^{-1} E, F_{1}, F_{2}\right\}$. If aw $(X)=2$, then $a_{02} \neq 0$ and $Q_{4} \notin U_{4}$, hence $X_{1}$ is Gorenstein. Otherwise $f_{1}$ satisfies the condition in (2) since $\left(g_{1}^{*} \Delta_{X}(k)-\right.$ $\left.\frac{1}{2} E\right)_{\mid U_{4}}=-\frac{k+2}{4} \operatorname{div}_{U_{4}}(u)$.

Remark 9.10. In (9.9), aw $(X)=2$ if and only if $l(X) \leq 2$. If $\operatorname{aw}(X)=$ 2 , then the condition $(* 4)$ does not hold.

The pair $\left(U,-\frac{k+2}{4} \operatorname{div}_{U}(u)\right)$ in $(9.9)(2)$ is of the form in (9.1) and (9.3). It is easy to see that $w_{i}(U)=w_{i+2}(X)-w_{2}(X)$ for all positive integers $i$ and $l(U)=l(X)-2$. We also see that the condition $(* i)$ holds for $U$ if and only if the condition $(* i+2)$ holds for $X$.

Lemma 9.11. Let $X, w_{i}(X), \Delta_{X}(k), \mathcal{H}_{X}$ be as in (9.1) and (9.3), and assume that $\bar{w}_{1}(X)=1, w_{2}(X)=4$ and that the condition $(* 2)$ holds. Then $\operatorname{aw}(X) \geq 3$ and one of the following holds:

(1) If $\operatorname{aw}(X)=3$, then there is a $K_{X}+\mathcal{H}_{X}$-crepant Gorenstein resolution $f_{1}: X_{1} \rightarrow X$ such that $\# \mathcal{E}\left(f_{1}\right)=5$ with

$$
\begin{aligned}
& \# \mathcal{E}\left(f_{1}, \Delta_{X}(k),(k+2) / 4\right)=2, \quad \# \mathcal{E}\left(f_{1}, \Delta_{X}(k),(k+4) / 4\right)=1 \\
& \# \mathcal{E}\left(f_{1}, \Delta_{X}(k),(k+2) / 2\right)=1, \quad \# \mathcal{E}\left(f_{1}, \Delta_{X}(k),(k+3) / 2\right)=1
\end{aligned}
$$


(2) If aw $(X) \geq 4$, then there is a $K_{X}+\mathcal{H}_{X}$-crepant birational morphism $f_{1}: X_{1} \rightarrow X$ such that $\# \mathcal{E}\left(f_{1}\right)=5$ with

$$
\begin{aligned}
& \# \mathcal{E}\left(f_{1}, \Delta_{X}(k),(k+2) / 4\right)=2 \text {, } \\
& \# \mathcal{E}\left(f_{1}, \Delta_{X}(k),(k+4) / 4\right)= \begin{cases}1 & \text { if } a_{11} \neq 0, \\
0 & \text { if } a_{11}=0,\end{cases} \\
& \# \mathcal{E}\left(f_{1}, \Delta_{X}(k),(k+2) / 2\right)=1 \text {, } \\
& \# \mathcal{E}\left(f_{1}, \Delta_{X}(k),(k+3) / 2\right)= \begin{cases}1 & \text { if } a_{11} \neq 0, \\
2 & \text { if } a_{11}=0,\end{cases}
\end{aligned}
$$

and that $X_{1}$ is Gorenstein terminal outside one point $P_{1}$. There is an open neighborhood $P_{1} \in U \subseteq X_{1}$ such that

$$
U \simeq\left\{\begin{array}{c}
x^{2}+y^{2} u+\left(2 \alpha z+b_{1} z^{3}\right) y-\frac{1}{4} h_{1}\left(z u^{1 / 2}\right)^{2} / u^{4} \\
-\frac{1}{2} b_{1} z^{3} h_{1}\left(z u^{1 / 2}\right) / u^{5 / 2}+g_{1}\left(z u^{1 / 2}, u\right) / u^{3}=0
\end{array}\right\} / \mathbb{Z}_{2}(1,1,1,0),
$$

where $\alpha^{2}=-a_{11}, g_{1}(z, u)=(g(z, u))_{\tau_{2}-w t \geq 6}$ and $h_{1}(z)=(h(z))_{\tau_{2}-w t \geq 8}$. We also have $a\left(G, X, \Delta_{X}(k)\right)=a\left(G, U,-\frac{k+\overline{2}}{4} \operatorname{div}_{U}(u)\right)$ for each prime divisor $G$ over $P_{1}$.

Proof. Since the condition $(* 2)$ holds, we see that $a_{02}=0$, hence $\operatorname{aw}(X) \geq 3$. We can write $\left(-\frac{1}{4} h(z)^{2}+g(z, u) u\right)_{\tau_{2}-w t=6}=-\left(\frac{1}{2} b_{1} z^{3}-\alpha z u\right)^{2}$, where $\alpha^{2}=-a_{11}$. With this notation, we see that there is an embedding $X \hookrightarrow(x, y, z, u, t) / \mathbb{Z}_{2}(1,1,1,0,1)$ such that

$$
X=\left\{\begin{array}{c}
x^{2}+(y-\alpha z) t+h_{1}(z) y+g_{1}(z, u)=0 \\
t=(y+\alpha z) u+b_{1} z^{3}
\end{array}\right\} / \mathbb{Z}_{2}(1,1,1,0,1),
$$

where $h_{1}(z)=(h(z))_{\tau_{2}-w t \geq 8}$ and $g_{1}(z, u)=(g(z, u))_{\tau_{2}-w t \geq 6}$. Let $g_{1}: Y_{1} \rightarrow X$ be the blow up with weight $(x, y, z, u, t)=\frac{1}{2}(3,1,1,2,5)$. By [Hay00, 5.9, 5.36], $g_{1}$ is divisorial with discrepancy $1 / 2$ and $Y_{1}$ is Gorenstein outside the origin $Q_{5}$ of the $t$-chart and the origin $Q_{4}$ of the $u$-chart. Let $E$ be the exceptional divisor of $g_{1}$.

We know that $Q_{5}$ has an open neighborhood $U_{5} \simeq(x, z, u) / \mathbb{Z}_{5}(3,1,2)$, $a\left(E, X, \Delta_{X}(k)\right)=(k+2) / 4$ and that

$$
\begin{aligned}
& \left(g_{1}^{*} \Delta_{X}(k)-\frac{1}{2} E\right)_{\mid U_{5}} \\
& \quad=-\frac{k}{4} \operatorname{div}_{U_{5}}(u)-\frac{k+2}{4} \operatorname{div}_{U_{5}}\left(\begin{array}{c}
x^{2} u-2 \alpha z u-b_{1} z^{3} \\
+\sum_{p+q=3} a_{p q} z^{2 p} u^{q+1}-\alpha b_{2} z^{6} u-b_{1} b_{2} z^{8}
\end{array}\right) .
\end{aligned}
$$


Let $h_{1}: X_{1} \rightarrow Y_{1}$ be the economic resolution of $Q_{5}$. Then we have $\mathcal{E}\left(h_{1}\right)=$ $\left\{F_{1}, \ldots, F_{4}\right\}$ with $a\left(F_{i}, Y_{1}\right)=i / 5(i=1, \ldots, 4)$. Thus, by $(2.10)$ and $(2.12)$, we have

$$
a\left(F_{i}, X, \Delta_{X}(k)\right)= \begin{cases}(k+2) / 4 & \text { if } i=1, \\ (k+2) / 2 & \text { if } i=2, \\ (k+4) / 4 & \text { if } i=3 \text { and " } a_{11} \neq 0 \text { or } a_{03} \neq 0 ", \\ (k+3) / 2 & \text { if " } i=3 \text { and } a_{11}=a_{03}=0 " \text { or } i=4 .\end{cases}
$$

On the other hand, the $u$-chart $U_{4}$ has an expression:

$$
U_{4}=\left\{\begin{array}{c}
x^{2}+(y-\alpha z) t+h_{1}\left(z u^{1 / 2}\right) / u^{5 / 2} \cdot y \\
\quad+g_{1}\left(z u^{1 / 2}, u\right) / u^{3}=0 \\
t u=y+\alpha z+b_{1} z^{3}
\end{array}\right\} / \mathbb{Z}_{2}(1,1,1,0,1)
$$

Let $f_{1}=g_{1} \circ h_{1}: X_{1} \rightarrow X$ be the composition. If $\operatorname{aw}(X)=3$, then $a_{03} \neq 0$ and $Q_{4} \notin U_{4}$, therefore $f_{1}$ is a Gorenstein resolution. Otherwise, $X_{1}$ is Gorenstein terminal outside $Q_{4}$ and $U_{4}$ is isomorphic to the given form of $U$. Since $\left(g_{1}^{*} \Delta_{X}(k)-\frac{1}{2} E\right)_{\mid U_{4}}=-\frac{k+2}{4} \operatorname{div}_{U_{4}}(u)$, we see that $a\left(G, X, \Delta_{X}(k)\right)=$ $a\left(G, U_{4}, \Delta_{U_{4}}(k+2)\right)$ for each prime divisor $G$ over $Q_{4}$

Remark 9.12. In (9.11), aw $(X)=3$ if and only if $l(X) \leq 4$. If $\operatorname{aw}(X)=$ 3 , then the condition $(* 4)$ does not hold. Since

The pair $\left(U,-\frac{k+2}{4} \operatorname{div}_{U}(u)\right)$ in $(9.11)(2)$ is of the form in (9.1) and (9.3).

$$
\begin{aligned}
& -\frac{1}{4}\left(2 \alpha z+b_{1} z^{3}\right)^{2}+\left(-\frac{1}{4} h_{1}\left(z u^{1 / 2}\right)^{2} / u^{4}-\frac{1}{2} b_{1} z^{3} h_{1}\left(z u^{1 / 2}\right) / u^{5 / 2}\right. \\
& \left.\quad+g_{1}\left(z u^{1 / 2}, u\right) / u^{3}\right) u \\
& =-\frac{1}{4} h\left(z u^{1 / 2}\right)^{2} / u^{3}+g\left(z u^{1 / 2}, u\right) / u^{2},
\end{aligned}
$$

we see that $w_{i}(U)=w_{i+2}(X)-w_{2}(X)-2$ for all positive integers $i$ and $l(U)=l(X)-2$. We also see that the condition $(* i)$ holds for $U$ if and only if the condition $(* i+2)$ holds for $X$. If $a_{11} \neq 0$, then $w_{1}(U)=-1$ and the condition $(* 4)$ holds for $X$.

Lemma 9.13. Let $\hat{X}, \hat{w}_{i}(\hat{X}), \hat{\Delta}_{\hat{X}}(k), \mathcal{H}_{\hat{X}}$ be as in (9.4) and (9.6), and assume that the condition $(\dagger 1)$ does not hold. Then one of the following holds: 
(1) If aw $(\hat{X})=\left(\hat{w}_{1}(\hat{X})+1\right) / 2$, then there is a $K_{\hat{X}}+\mathcal{H}_{\hat{X}}$-crepant Gorenstein resolution $f_{1}: X_{1} \rightarrow \hat{X}$ such that $\# \mathcal{E}\left(f_{1}\right)=\hat{w}_{1}(\hat{X})+2$ with

$$
\begin{aligned}
& \# \mathcal{E}\left(f_{1}, \hat{\Delta}_{\hat{X}}(k),(k+1) / 4\right)=1, \quad \# \mathcal{E}\left(f_{1}, \hat{\Delta}_{\hat{X}}(k),(k+3) / 4\right)=1, \\
& \# \mathcal{E}\left(f_{1}, \hat{\Delta}_{\hat{X}}(k),(k+1) / 2\right)=\left(\hat{w}_{1}(\hat{X})+1\right) / 2, \\
& \# \mathcal{E}\left(f_{1}, \hat{\Delta}_{\hat{X}}(k),(k+2) / 2\right)=\left(\hat{w}_{1}(\hat{X})-1\right) / 2 .
\end{aligned}
$$

(2) If $\operatorname{aw}(\hat{X}) \geq\left(\hat{w}_{1}(\hat{X})+3\right) / 2$, then there is a $K_{\hat{X}}+\mathcal{H}_{\hat{X}}$-crepant birational morphism $f_{1}: X_{1} \rightarrow \hat{X}$ such that $\# \mathcal{E}\left(f_{1}\right)=\hat{w}_{1}(\hat{X})+2$ with

$$
\begin{aligned}
& \# \mathcal{E}\left(f_{1}, \hat{\Delta}_{\hat{X}}(k),(k+1) / 4\right)=1, \\
& \# \mathcal{E}\left(f_{1}, \hat{\Delta}_{\hat{X}}(k),(k+1) / 2\right)=\left(\hat{w}_{1}(\hat{X})+1\right) / 2, \\
& \# \mathcal{E}\left(f_{1}, \hat{\Delta}_{\hat{X}}(k),(k+2) / 2\right)=\left(\hat{w}_{1}(\hat{X})+1\right) / 2,
\end{aligned}
$$

and that $X_{1}$ is Gorenstein terminal outside one point $P_{1}$. There is an open neighborhood $P_{1} \in U \subseteq X_{1}$ such that

$$
U \simeq\left\{x^{2}+y^{2} u+f\left(z u^{1 / 4}, u^{1 / 2}\right) / u^{\hat{w}_{1}(\hat{X}) / 2}=0\right\} / \mathbb{Z}_{2}(1,1,1,0)
$$

Furthermore, we have $a\left(G, \hat{X}, \hat{\Delta}_{\hat{X}}(k)\right)=a\left(G, U,-\frac{k+1}{4} \operatorname{div}_{U}(u)\right)$ for each prime divisor $G$ over $P_{1}$.

Proof. As we can treat the case $\hat{w}_{1} \equiv 3(\bmod 4)$ similarly, we shall assume that $\hat{w}_{1} \equiv 1(\bmod 4)$ in the following. Let $g_{1}: Y_{1} \rightarrow \hat{X}$ be the blow up with weight $(x, y, z, u)=\frac{1}{4}\left(\hat{w}_{1}, \hat{w}_{1}+2,1,2\right)$. By [Hay99, 7.4], $g_{1}$ is divisorial with discrepancy $1 / 4$ and $Y_{1}$ is Gorenstein outside the origin $Q_{2}$ of the $y$-chart and the origin $Q_{4}$ of the $u$-chart. Let $E$ be the exceptional divisor of $g_{1}$.

The point $Q_{2}$ has an open neighborhood $U_{2} \simeq(x, z, u) / \mathbb{Z}_{\hat{w}_{1}+2}(-2,1,2)$, $a\left(E, \hat{X}, \hat{\Delta}_{\hat{X}}(k)\right)=(k+1) / 4$ and that

$$
\left(g_{1}^{*} \hat{\Delta}_{\hat{X}}(k)-\frac{1}{4} E\right)_{\mid U_{2}}=-\frac{k}{2} \operatorname{div}_{U_{2}}(u)-\frac{k+1}{4} \operatorname{div}_{U_{2}}\left(x^{2}+(f(z, u))_{\rho-w t=\hat{w}_{1}}\right) .
$$

Let $h_{1}: X_{1} \rightarrow Y_{1}$ be the economic resolution of $Q_{1}$. Then we have $\mathcal{E}\left(h_{1}\right)=$ $\left\{F_{1}, \ldots, F_{\hat{w}_{1}+1}\right\}$ with $a\left(F_{i}, Y_{1}\right)=i /\left(\hat{w}_{1}+2\right)\left(i=1, \ldots, \hat{w}_{1}+1\right)$. Thus, by 
(2.10) and (2.12), we have

$$
\begin{aligned}
a & \left(F_{i}, \hat{X}, \hat{\Delta}_{\hat{X}}(k)\right) \\
& =a\left(F_{i}, Y_{1}, g_{1}^{*} \hat{\Delta}_{\hat{X}}(k)-\frac{1}{4} E\right) \\
& =a\left(F_{i}, U_{1},-\frac{k}{2} \operatorname{div}_{U_{1}}(u)-\frac{k+1}{4} \operatorname{div}_{U_{1}}\left(y^{2}+(f(z, u))_{\rho-w t=\hat{w}_{1}}\right)\right) \\
& = \begin{cases}(k+1) / 2 & \text { if } i=1, \ldots,\left(\hat{w}_{1}+1\right) / 2, \\
(k+3) / 4 & \text { if } i=\left(\hat{w}_{1}+3\right) / 2 \text { and } c_{0, \hat{w}_{1}} \neq 0 \\
(k+2) / 2 & \text { " } i=\left(\hat{w}_{1}+3\right) / 2 \text { and } c_{0, \hat{w}_{1}}=0 " \text { or } i \geq\left(\hat{w}_{1}+5\right) / 2 .\end{cases}
\end{aligned}
$$

On the other hand, we can write the $u$-chart $U_{4}$ as follows:

$$
U_{4}=\left\{x^{2}+y^{2} u+f\left(z u^{1 / 4}, u^{1 / 2}\right) / u^{\hat{w}_{1} / 2}=0\right\} / \mathbb{Z}_{2}(1,1,1,0) .
$$

Let $f_{1}=g_{1} \circ h_{1}: X_{1} \rightarrow \hat{X}$ be the composition. If $\operatorname{aw}(\hat{X})=\left(\hat{w}_{1}+1\right) / 2$, then $Q_{4} \notin U_{4}$ and $f_{1}$ is a Gorenstein resolution. Otherwise, $X_{1}$ is Gorenstein terminal outside $Q_{4}$. Since $\left(g_{1}^{*} \hat{\Delta}_{\hat{X}}(k)-\frac{1}{4} E\right)_{\mid U_{4}}=-\frac{k+1}{4} \operatorname{div}_{U_{4}}(u)$, we have $a\left(G, \hat{X}, \hat{\Delta}_{\hat{X}}(k)\right)=a\left(G, U_{4},-\frac{k+1}{4} \operatorname{div}_{U_{4}}(u)\right)$ for each prime divisor $G$ over $Q_{4}$.

Remark 9.14. In (9.13), aw $(\hat{X})=\left(\hat{w}_{1}(\hat{X})+1\right) / 2$ if and only if $\hat{l}(\hat{X})=$ 1. If $\operatorname{aw}(\hat{X})=\left(\hat{w}_{1}(\hat{X})+1\right) / 2$, then the condition $(\dagger 3)$ does not hold.

The pair $\left(U,-\frac{k+1}{4} \operatorname{div}_{U}(u)\right)$ in $(9.13)(2)$ is of the form in (9.1) and (9.3). It is easy to see that $w_{i}(U)=\hat{w}_{i+1}(\hat{X})-\hat{w}_{1}(\hat{X})$ for all positive integers $i$ and $l(U)=\hat{l}(\hat{X})-1$. We also see that the condition $(* i)$ holds for $U$ if and only if the condition $(\dagger i+1)$ holds for $\hat{X}$.

Lemma 9.15. Let $\hat{X}, \hat{w}_{i}(\hat{X}), \hat{\Delta}_{\hat{X}}(k), \mathcal{H}_{\hat{X}}$ be as in (9.4) and (9.6), and assume that the condition $(\dagger 1)$ hold. Then $\mathrm{aw}(\hat{X}) \geq\left(\hat{w}_{1}(\hat{X})+3\right) / 2$ and one of the following holds:

(1) If $\operatorname{aw}(\hat{X})=\left(\hat{w}_{1}(\hat{X})+3\right) / 2$, then there is a $K_{\hat{X}}+\mathcal{H}_{\hat{X}}$ crepant Gorenstein resolution $f_{1}: X_{1} \rightarrow \hat{X}$ such that $\# \mathcal{E}\left(f_{1}\right)=\hat{w}_{1}(\hat{X})+4$ with

$$
\begin{aligned}
& \# \mathcal{E}\left(f_{1}, \hat{\Delta}_{\hat{X}}(k),(k+1) / 4\right)=2, \quad \# \mathcal{E}\left(f_{1}, \hat{\Delta}_{\hat{X}}(k),(k+3) / 4\right)=1 \\
& \# \mathcal{E}\left(f_{1}, \hat{\Delta}_{\hat{X}}(k),(k+1) / 2\right)=\left(\hat{w}_{1}(\hat{X})+1\right) / 2 \\
& \# \mathcal{E}\left(f_{1}, \hat{\Delta}_{\hat{X}}(k),(k+2) / 2\right)=\left(\hat{w}_{1}(\hat{X})+1\right) / 2
\end{aligned}
$$


(2) If $\operatorname{aw}(\hat{X}) \geq\left(\hat{w}_{1}(\hat{X})+5\right) / 2$, then there is a $K_{\hat{X}}+\mathcal{H}_{\hat{X}}$-crepant birational morphism $f_{1}: X_{1} \rightarrow \hat{X}$ such that $\# \mathcal{E}\left(f_{1}\right)=\hat{w}_{1}(\hat{X})+4$ with

$$
\begin{aligned}
& \# \mathcal{E}\left(f_{1}, \hat{\Delta}_{\hat{X}}(k),(k+1) / 4\right)=2, \\
& \# \mathcal{E}\left(f_{1}, \hat{\Delta}_{\hat{X}}(k),(k+3) / 4\right)= \begin{cases}1 & \text { if } c_{1, \hat{w}_{1}-1} \neq 0, \\
0 & \text { if } c_{1, \hat{w}_{1}-1}=0,\end{cases} \\
& \# \mathcal{E}\left(f_{1}, \hat{\Delta}_{\hat{X}}(k),(k+1) / 2\right)=\left(\hat{w}_{1}(\hat{X})+1\right) / 2, \\
& \# \mathcal{E}\left(f_{1}, \hat{\Delta}_{\hat{X}}(k),(k+2) / 2\right)= \begin{cases}\left(\hat{w}_{1}(\hat{X})+1\right) / 2 & \text { if } c_{1, \hat{w}_{1}-1} \neq 0, \\
\left(\hat{w}_{1}(\hat{X})+3\right) / 2 & \text { if } c_{1, \hat{w}_{1}-1}=0 .\end{cases}
\end{aligned}
$$

Moreover $X_{1}$ is Gorenstein terminal outside one point $P_{1}$, and there is an open neighborhood $P_{1} \in U \subseteq X_{1}$ such that

$U \simeq\left\{x^{2}+y^{2} u+2 g(z, 1) y+h\left(z u^{1 / 4}, u^{1 / 2}\right) / u^{\left(\hat{w}_{1}(\hat{X})+2\right) / 2}=0\right\} / \mathbb{Z}_{2}(1,1,1,0)$,

where $g(z, u)^{2}=-(f(z, u))_{\rho_{1}-w t=\hat{w}_{1}(X)}, h(z, u)=(f(z, u))_{\rho_{1}-w t \geq \hat{w}_{1}(X)+2}$. We have $a\left(G, \hat{X}, \hat{\Delta}_{\hat{X}}(k)\right)=a\left(G, U,-\frac{k+1}{4} \operatorname{div}_{U}(u)\right)$ for each prime divisor $G$ over $P_{1}$.

Proof. Since the condition $(\dagger 1)$ holds, we see that $c_{0, \hat{w}_{1}}=0$, hence $\operatorname{aw}(\hat{X}) \geq\left(\hat{w}_{1}+3\right) / 2$. We also see that there is an embedding $X \hookrightarrow$ $(x, y, z, u) / \mathbb{Z}_{4}(1,3,1,2)$ such that

$$
X=\left\{\begin{aligned}
\left\{x^{2}+y^{2}+2 g(z, u) x+h(z, u)=0\right\} / \mathbb{Z}_{4}(1,3,1,2) & \\
& \text { if } \hat{w}_{1} \equiv 1(\bmod 4) \\
\left\{x^{2}+y^{2}+2 g(z, u) y+h(z, u)=0\right\} / \mathbb{Z}_{4}(1,3,1,2) & \text { if } \hat{w}_{1} \equiv 3(\bmod 4)
\end{aligned}\right.
$$

where $g(z, u)^{2}=-(f(z, u))_{\rho_{1}-w t=\hat{w}_{1}}, h(z, u)=(f(z, u))_{\rho_{1}-w t \geq \hat{w}_{1}+2}$. Since we can treat the case $\hat{w}_{1} \equiv 3(\bmod 4)$ similarly, we shall assume that $\hat{w}_{1} \equiv 1$ $(\bmod 4)$ in the following. Let $g_{1}: Y_{1} \rightarrow \hat{X}$ be the blow up with weight $(x, y, z, u)=\frac{1}{4}\left(\hat{w}_{1}+4, \hat{w}_{1}+2,1,2\right)$. By [Hay99, 7.9], $g_{1}$ is divisorial with discrepancy $1 / 4$ and $Y_{1}$ is Gorenstein outside the origin $Q_{1}$ of the $x$-chart and the origin $Q_{4}$ of the $u$-chart. Let $E$ be the exceptional divisor of $g_{1}$.

The point $Q_{1}$ has an open neighborhood $U_{1} \simeq(y, z, u) / \mathbb{Z}_{\hat{w}_{1}+4}(-2,1,2)$, $a\left(E, \hat{X}, \hat{\Delta}_{\hat{X}}(k)\right)=(k+1) / 4$ and that

$$
\begin{aligned}
& \left(g_{1}^{*} \hat{\Delta}_{\hat{X}}(k)-\frac{1}{4} E\right)_{\mid U_{1}} \\
& \quad=-\frac{k}{2} \operatorname{div}_{U_{1}}(u)-\frac{k+1}{4} \operatorname{div}_{U_{1}}\left(y^{2}+2 g(z, u)+(f(z, u))_{\rho-w t=\hat{w}_{1}+2}\right) .
\end{aligned}
$$


Let $h_{1}: X_{1} \rightarrow Y_{1}$ be the economic resolution of $Q_{1}$. Then we have $\mathcal{E}\left(h_{1}\right)=$ $\left\{F_{1}, \ldots, F_{\hat{w}_{1}+3}\right\}$ with $a\left(F_{i}, Y_{1}\right)=i /\left(\hat{w}_{1}+4\right)\left(i=1, \ldots, \hat{w}_{1}+3\right)$. Thus, by (2.10) and (2.12), we have

$$
\begin{aligned}
& a\left(F_{i}, \hat{X}, \hat{\Delta}_{\hat{X}}(k)\right) \\
& =a\left(F_{i}, Y_{1}, g_{1}^{*} \hat{\Delta}_{\hat{X}}(k)-\frac{1}{4} E\right) \\
& =a\left(F_{i}, U_{1},-\frac{k}{2} \operatorname{div}_{U_{1}}(u)-\frac{k+1}{4} \operatorname{div}_{U_{1}}\left(y^{2}+2 g(z, u)+(f(z, u))_{\rho-w t=\hat{w}_{1}+2}\right)\right) \\
& \quad= \begin{cases}(k+1) / 4 & \text { if } i=1, \\
(k+1) / 2 & \text { if } i=2, \ldots,\left(\hat{w}_{1}+3\right) / 2, \\
(k+3) / 4 & \text { if } i=\left(\hat{w}_{1}+5\right) / 2 \text { and " } c_{1, \hat{w}_{1}-1} \neq 0 \text { or } c_{0, \hat{w}_{1}+2} \neq 0 ", \\
(k+2) / 2 & \text { otherwise. }\end{cases}
\end{aligned}
$$

On the other hand, the $u$-chart $U_{4}$ has an expression:

$$
U_{4}=\left\{x^{2}+y^{2} u+2 g(z, 1) y+h\left(z u^{1 / 4}, u^{1 / 2}\right) / u^{\left(\hat{w}_{1}+2\right) / 2}=0\right\} / \mathbb{Z}_{2}(1,1,1,0) .
$$

Let $f_{1}=g_{1} \circ h_{1}: X_{1} \rightarrow \hat{X}$ be the composition. If $\operatorname{aw}(\hat{X})=\left(\hat{w}_{1}+3\right) / 2$, then $Q_{4} \notin U_{4}$ and $f_{1}$ is a Gorenstein resolution. Otherwise, $X_{1}$ is Gorenstein terminal outside $Q_{4}$. Since $\left(g_{1}^{*} \hat{\Delta}_{\hat{X}}(k)-\frac{1}{4} E\right)_{\mid U_{4}}=-\frac{k+1}{4} \operatorname{div}_{U_{4}}(u)$, we have $a\left(G, \hat{X}, \hat{\Delta}_{\hat{X}}(k)\right)=a\left(G, U_{4},-\frac{k+1}{4} \operatorname{div}_{U_{4}}(u)\right)$ for each prime divisor $G$ over $Q_{4}$.

Remark 9.16. In (9.15), aw $(\hat{X})=\left(\hat{w}_{1}(\hat{X})+3\right) / 2$ if and only if $\hat{l}(\hat{X}) \leq$ 3. If aw $(\hat{X})=\left(\hat{w}_{1}(\hat{X})+3\right) / 2$, then the condition $(\dagger 3)$ does not hold. Since

The pair $\left(U,-\frac{k+1}{4} \operatorname{div}_{U}(u)\right)$ in $(9.15)(2)$ is of the form in (9.1) and (9.3).

$$
-\frac{1}{4}(2 g(z, 1))^{2}+\left(h\left(z u^{1 / 4}, u^{1 / 2}\right) / u^{\left(\hat{w}_{1}+2\right) / 2}\right) u=f\left(z u^{1 / 4}, u^{1 / 2}\right) / u^{\hat{w}_{1} / 2},
$$

we have $w_{i}(U)=\hat{w}_{i+1}(\hat{X})-\hat{w}_{1}(\hat{X})-2$ for all positive integers $i$ and $l(U)=$ $\hat{l}(\hat{X})-1$. We also see that the condition $(* i)$ holds for $U$ if and only if the condition $(\dagger i+1)$ holds for $\hat{X}$. If $c_{1, \hat{w}_{1}-1} \neq 0$, then $w_{1}(U)=-1$ and the condition $(\dagger 3)$ holds for $\hat{X}$.

Lemma 9.17. Let $X, w_{i}(X), \Delta_{X}(k), \mathcal{H}_{X}$ be as in (9.1) and (9.3), and assume that $w_{1}(X) \geq 3$. Then there is a $K_{X}+\mathcal{H}_{X}$-crepant birational morphism $f_{1}: X_{1} \rightarrow X$ such that $\# \mathcal{E}\left(f_{1}\right)=\bar{w}_{1}(X)-2$ with

$$
\begin{aligned}
& \# \mathcal{E}\left(f_{1}, \Delta_{X}(k),(k+1) / 2\right)=\left(\bar{w}_{1}(X)-1\right) / 2, \\
& \# \mathcal{E}\left(f_{1}, \Delta_{X}(k),(k+2) / 2\right)=\left(\bar{w}_{1}(X)-3\right) / 2,
\end{aligned}
$$


and that $X_{1}$ is Gorenstein terminal outside one point $P_{1}$. There is an open neighborhood $P_{1} \in \hat{U} \subseteq X_{1}$ such that

$$
\hat{U} \simeq\left\{\begin{array}{c}
x^{2}+y^{2}-\frac{1}{4} h\left(z u^{1 / 2}\right)^{2} / u^{\bar{w}_{1}(X)+2} \\
+g\left(z u^{1 / 2}, u^{2}\right) / u^{\bar{w}_{1}(X)}=0
\end{array}\right\} / \mathbb{Z}_{4}(1,3,1,2) .
$$

Furthermore, we have $a\left(G, X, \Delta_{X}(k)\right)=a\left(G, \hat{U},-\frac{k+1}{2} \operatorname{div}_{\hat{U}}(u)\right)$ for each prime divisor $G$ over $P_{1}$.

Proof. Let $g_{1}: Y_{1} \rightarrow X$ be the blow up with weight $(x, y, z, u)=$ $\frac{1}{2}\left(\bar{w}_{1}, \bar{w}_{1}-2,1,4\right)$. By [Hay00, 5.4], $g_{1}$ is divisorial with discrepancy $1 / 2$ and $Y_{1}$ is Gorenstein outside the origin $Q_{2}$ of the $y$-chart and the origin $Q_{4}$ of the $u$-chart. Let $E$ be the exceptional divisor of $g_{1}$.

The point $Q_{2}$ has an open neighborhood $U_{2} \simeq(x, y, z) / \mathbb{Z}_{\bar{w}_{1}-2}(2,-2,1)$, $a\left(E, X, \Delta_{X}(k)\right)=(k+1) / 2$ and that

$$
\begin{aligned}
& \left(g_{1}^{*} \Delta_{X}(k)-\frac{1}{2} E\right)_{\mid U_{2}} \\
& \quad=-\frac{k}{4} \operatorname{div}_{U_{2}}\left(x^{2}+h\left(z y^{1 / 2}\right) / y^{\left(\bar{w}_{1}+2\right) / 2}+g\left(z y^{1 / 2}, 0\right) / y^{\bar{w}_{1}}\right)-\frac{k+1}{2} \operatorname{div}_{U_{2}}(y) .
\end{aligned}
$$

Let $h_{1}: X_{1} \rightarrow Y_{1}$ be the economic resolution of $Q_{2}$ and let $\mathcal{E}\left(h_{1}\right)=$ $\left\{F_{1}, \ldots, F_{\bar{w}_{1}-3}\right\}$ with $a\left(F_{i}, Y_{1}\right)=i /\left(\bar{w}_{1}-2\right)\left(i=1, \ldots, \bar{w}_{1}-3\right)$. Then we have

$$
\begin{aligned}
a( & \left.F_{i}, X, \Delta_{X}(k)\right) \\
= & a\left(F_{i}, Y_{1}, g_{1}^{*} \Delta_{X}(k)-\frac{1}{2} E\right) \\
= & a\left(F_{i}, U_{2},-\frac{k}{4} \operatorname{div}_{U_{2}}\left(x^{2}+h\left(z y^{1 / 2}\right) / y^{\left(\bar{w}_{1}+2\right) / 2}+g\left(z y^{1 / 2}, 0\right) / y^{\bar{w}_{1}}\right)\right. \\
& \left.\quad-\frac{k+1}{2} \operatorname{div}_{U_{2}}(y)\right) \\
= & \begin{cases}(k+1) / 2 & \text { if } i=1, \ldots,\left(\bar{w}_{1}-3\right) / 2 \\
(k+2) / 2 & \text { if } i=\left(\bar{w}_{1}-1\right) / 2, \ldots, \bar{w}_{1}-3 .\end{cases}
\end{aligned}
$$

On the other hand, the $u$-chart $U_{4}$ has an expression:

$U_{4}=\left\{x^{2}+y^{2}+h\left(z u^{1 / 2}\right) / u^{\left(\bar{w}_{1}+2\right) / 2} \cdot y+g\left(z u^{1 / 2}, u^{2}\right) / u^{\bar{w}_{1}}=0\right\} / \mathbb{Z}_{4}(1,3,1,2)$,

which is isomorphic to the given form of $U$. We also know that $\left(g_{1}^{*} \Delta_{X}(k)-\right.$ $\left.\frac{1}{2} E\right)_{\mid U_{4}}=-\frac{k+1}{2} \operatorname{div}_{U_{4}}(u)$. Hence $a\left(G, X, \Delta_{X}(k)\right)=a\left(G, U_{4},-\frac{k+1}{2} \operatorname{div}_{U_{4}}(u)\right)$ for each prime divisor $G$ over $Q_{4}$. 
Remark 9.18. The pair $\left(\hat{U},-\frac{k+1}{2} \operatorname{div}_{\hat{U}}(u)\right)$ in (9.17) is of the form in (9.4) and (9.6). We see that $\hat{w}_{i}(\hat{U})=w_{i+1}(X)-\bar{w}_{1}(X)$ for all positive integers $i$ and $\hat{l}(\hat{U})=l(X)-1$. We also see that the condition $(\dagger i)$ holds for $\hat{U}$ if and only if the condition $(* i+1)$ holds for $X$.

Now we state our main results in this section. The following theorem (9.19) (resp. (9.20)) relates the number of exceptional prime divisors of a Gorenstein resolution with the data $w_{i}(X)$ and conditions $(* i)$ (resp. $\hat{w}_{i}(\hat{X})$ and conditions $\left.(\dagger i)\right)$. Though it should be possible to write down all informations on exceptional prime divisors obtained from (9.7)-(9.18), our results are restricted to divisors with discrepancies $\leq 1$ over $X$ and $\hat{X}$. This is because we have to divide into many cases. Proofs of (9.19) and (9.20) will be given at the same time.

Theorem 9.19. Let $X, w_{i}(X), \Delta_{X}(k), \mathcal{H}_{X}$ be as in (9.1) and (9.3), and assume that $X$ is of type $(\mathrm{cD} / 2)$. Then there is a $K_{X}+\mathcal{H}_{X}$-crepant Gorenstein resolution $f: Y \rightarrow X$ such that $\# \mathcal{E}(f) \leq 2 \mathrm{aw}(X)$ with

$$
\begin{aligned}
& \# \mathcal{E}\left(f, \Delta_{X}(k),(k+2) / 4\right)= \begin{cases}1 & \text { if }(* 2) \text { does not hold }, \\
2 & \text { if }(* 2) \text { holds },\end{cases} \\
& \# \mathcal{E}\left(f, \Delta_{X}(k),(k+4) / 4\right)= \begin{cases}1 & \text { if }(* 4) \text { does not hold, } \\
2 & \text { if }(* 4) \text { holds, }\end{cases} \\
& \# \mathcal{E}\left(f, \Delta_{X}(k),(k+1) / 2\right)=\left(\bar{w}_{1}(X)-1\right) / 2 \text {, } \\
& \# \mathcal{E}\left(f, \Delta_{X}(k),(k+2) / 2\right)=w_{2}(X) / 2-1,
\end{aligned}
$$

and other $E \in \mathcal{E}(f)$ satisfies $a\left(E, X, \Delta_{X}(k)\right) \geq(k+6) / 4$ or $\geq(k+3) / 2$.

TheOREM 9.20. Let $\hat{X}, \hat{w}_{i}(\hat{X}), \hat{\Delta}_{\hat{X}}(k), \mathcal{H}_{\hat{X}}$ be as in (9.4) and (9.6). Then there is a $K_{\hat{X}}+\mathcal{H}_{\hat{X}}$-crepant Gorenstein resolution $f: \hat{Y} \rightarrow \hat{X}$ such that $\# \mathcal{E}(f) \leq 2 \operatorname{aw}(\hat{X})+1$ with

$$
\begin{aligned}
& \# \mathcal{E}\left(f, \hat{\Delta}_{\hat{X}}(k),(k+1) / 4\right)= \begin{cases}1 & \text { if }(\dagger 1) \text { does not hold }, \\
2 & \text { if }(\dagger 1) \text { holds, }\end{cases} \\
& \# \mathcal{E}\left(f, \hat{\Delta}_{\hat{X}}(k),(k+3) / 4\right)= \begin{cases}1 & \text { if }(\dagger 3) \text { does not hold, } \\
2 & \text { if }(\dagger 3) \text { holds, }\end{cases} \\
& \# \mathcal{E}\left(f, \hat{\Delta}_{\hat{X}}(k),(k+1) / 2\right)=\left(\hat{w}_{1}(\hat{X})+1\right) / 2, \\
& \# \mathcal{E}\left(f, \hat{\Delta}_{\hat{X}}(k),(k+2) / 2\right)=\left\lfloor\hat{w}_{2}(\hat{X}) / 2\right\rfloor,
\end{aligned}
$$


and other $E \in \mathcal{E}(f)$ satisfies a $\left(E, \hat{X}, \hat{\Delta}_{\hat{X}}(k)\right) \geq(k+3) / 2$ or $\geq(k+5) / 4$.

Proof of (9.19) and (9.20). (A) If $w_{2}(X) \leq 2$, then it follows from (9.7) and (9.8) that there is a Gorenstein resolution $f: Y \rightarrow X$ such that $\# \mathcal{E}(f) \leq 2 \operatorname{aw}(X)$. This statement is not a part of (9.19) since $X$ is not of type $(\mathrm{cD} / 2)$, but we need this in the following.

(B) We shall prove (9.20) when the condition $(\dagger 1)$ does not hold and $\hat{l}(\hat{X}) \leq 1$, or the condition $(\dagger 1)$ holds and $\hat{l}(\hat{X}) \leq 3$.

In the first case, we have aw $(\hat{X})=\left(\hat{w}_{1}(\hat{X})+1\right) / 2$ by $(9.14)$, hence there is a $K_{\hat{X}}+\mathcal{H}_{\hat{X}}$-crepant Gorenstein resolution $f_{1}: X_{1} \rightarrow \hat{X}$ as in (9.13)(1). Since $\# \mathcal{E}\left(f_{1}\right)=\hat{w}_{1}(\hat{X})+2=2 \operatorname{aw}(\hat{X})+1,\left\lfloor\hat{w}_{2}(\hat{X}) / 2\right\rfloor=\left(\hat{w}_{1}(\hat{X})-1\right) / 2$ and since the condition $(\dagger 3)$ does not hold, this $f_{1}$ satisfies the conditions in $(9.20)$.

In the second case, we can take a $K_{\hat{X}}+\mathcal{H}_{\hat{X}}$-crepant Gorenstein resolution $f_{1}: X_{1} \rightarrow \hat{X}$ as in (9.15)(1). Then we again see that $f_{1}$ satisfies the conditions in (9.20) by a similar argument as above.

(C) We shall prove $(9.19)$ when the condition $(* 2)$ does not hold and $l(X) \leq 2$. Since $X$ is of type $(\mathrm{cD} / 2)$, we have either $\bar{w}_{1}(X)=1$ and $w_{2}(X)=4$, or $\bar{w}_{1}(X) \geq 3$.

If $\bar{w}_{1}(X)=1$ and $w_{2}(X)=4$, then there is a $K_{X}+\mathcal{H}_{X}$-crepant Gorenstein resolution $f_{1}: X_{1} \rightarrow X$ as in $(9.9)(1)$. Since $\# \mathcal{E}\left(f_{1}\right)=3 \leq 4=$ 2aw $(X),\left(w_{1}(X)-1\right) / 2=0$ and since $w_{2}(X) / 2-1=1$, we see that $f_{1}$ satisfies the conditions in (9.19).

If $\bar{w}_{1}(X) \geq 3$, then we first take $f_{1}: X_{1} \rightarrow X$ as in (9.17). Since $\hat{U}$ in (9.17) satisfies $\hat{l}(\hat{U}) \leq 1$, there is a $K_{X_{1}}+\mathcal{H}_{X_{1}}$-crepant Gorenstein resolution $g_{1}: Y \rightarrow X_{1}$ as in (9.20) by using the results in (B). Let $f=f_{1} \circ g_{1}: Y \rightarrow X$ be the composition. This is a $K_{X}+\mathcal{H}_{X}$-crepant Gorenstein resolution. We see that $\# \mathcal{E}(f)=\# \mathcal{E}\left(f_{1}\right)+\# \mathcal{E}\left(g_{1}\right) \leq\left(\bar{w}_{1}(X)-2\right)+(2 \mathrm{aw}(\hat{U})+1)=2 \mathrm{aw}(X)$. By (9.17) and (9.18), we also see that

$$
\begin{aligned}
\# \mathcal{E}\left(f, \Delta_{X}(k),(k+2) / 4\right)=\# \mathcal{E}\left(g_{1}, \Delta_{\hat{U}}(k),(k+1) / 4\right)=1, \\
\# \mathcal{E}\left(f, \Delta_{X}(k),(k+4) / 4\right)=\# \mathcal{E}\left(g_{1}, \Delta_{\hat{U}}(k),(k+3) / 4\right)=1, \\
\# \mathcal{E}\left(f, \Delta_{X}(k),(k+1) / 2\right)=\left(\bar{w}_{1}(X)-1\right) / 2, \\
\# \mathcal{E}\left(f, \Delta_{X}(k),(k+2) / 2\right)=\# \mathcal{E}\left(f_{1}, \Delta_{X}(k),(k+2) / 2\right) \\
\quad+\# \mathcal{E}\left(g_{1}, \Delta_{\hat{U}}(k),(k+1) / 2\right) \\
=\left(\bar{w}_{1}(X)-3\right) / 2+\left(w_{2}(X)-\bar{w}_{1}(X)+1\right) / 2 \\
=w_{2}(X) / 2-1,
\end{aligned}
$$


and other $E \in \mathcal{E}(f)$ satisfies $a\left(E, X, \Delta_{X}(k)\right) \geq(k+6) / 4$ or $\geq(k+3) / 2$. Since the condition $(* 4)$ does not hold in this case, $f: Y \rightarrow X$ satisfies the condition in (9.19).

(D) By a similar way, we can prove $(9.19)$ when the condition $(* 2)$ holds and $l(X) \leq 4$.

Let $l$ be a positive integer, and assume that (9.20) holds for all $\hat{X}$ with $\hat{l}(\hat{X}) \leq l-1$ and (9.19) holds for all $X$ with $l(X) \leq l-1$.

(E) We shall prove (9.19) for $X$ with $l(X)=l$. We first assume that the condition $(* 2)$ does not hold for $X$. By the result in $(\mathrm{C})$, we may assume that $l=l(X) \geq 3$.

If $\bar{w}_{1}(X)=1$ and $w_{2}(X)=4$, then we first take $f_{1}: X_{1} \rightarrow X$ as in $(9.9)(2)$. Since $U$ in $(9.9)(2)$ satisfies $\bar{w}_{1}(U)=1, w_{2}(U) \leq 4$ and $l(U)=l-2$, there is a $K_{X_{1}}+\mathcal{H}_{X_{1}}$-crepant Gorenstein resolution $g_{1}: Y \rightarrow X_{1}$ as in (9.19) of (9.8). Let $f=f_{1} \circ g_{1}: Y \rightarrow X$ be the composition. We see that $\# \mathcal{E}(f)=\# \mathcal{E}\left(f_{1}\right)+\# \mathcal{E}\left(g_{1}\right) \leq 3+2 \operatorname{aw}(U)=3+(2 \mathrm{aw}(X)-4) \leq 2 \mathrm{aw}(X)$. We also see that

$$
\begin{aligned}
\# \mathcal{E}\left(f, \Delta_{X}(k),(k+2) / 4\right) & =\# \mathcal{E}\left(f_{1}, \Delta_{X}(k),(k+2) / 4\right)=1, \\
\# \mathcal{E}\left(f, \Delta_{X}(k),(k+4) / 4\right)=\# \mathcal{E}\left(g_{1}, \Delta_{U}(k),(k+2) / 4\right) & \\
& = \begin{cases}1 & \text { if }(* 4) \text { does not hold, } \\
2 & \text { if }(* 4) \text { holds, }\end{cases} \\
\# \mathcal{E}\left(f, \Delta_{X}(k),(k+1) / 2\right)=\# \mathcal{E}\left(f_{1}, \Delta_{X}(k),(k+1) / 2\right)=0, & \# \mathcal{E}\left(f, \Delta_{X}(k),(k+2) / 2\right)=\# \mathcal{E}\left(f_{1}, \Delta_{X}(k),(k+2) / 2\right)=1,
\end{aligned}
$$

and other $E \in \mathcal{E}(f)$ satisfies $a\left(E, X, \Delta_{X}(k)\right) \geq(k+6) / 4$ or $\geq(k+3) / 2$. Hence $f: Y \rightarrow X$ satisfies the conditions in (9.19).

If $\bar{w}_{1}(X) \geq 3$, then as in the proof of $(\mathrm{C})$, we first take $f_{1}: X_{1} \rightarrow X$ as in (9.17) and a Gorenstein resolution $g_{1}: Y \rightarrow X_{1}$ as in (9.20) which is obtained by induction hypothesis. Then the composition $f=f_{1} \circ g_{1}: Y \rightarrow$ $X$ is a $K_{X}+\mathcal{H}_{X}$-crepant Gorenstein resolution with required properties.

If the condition $(* 2)$ holds, then we can prove $(9.19)$ similarly by using induction and (9.7).

(F) Finally we shall prove (9.20) for $X$ with $\hat{l}(\hat{X})=l$.

We first assume that the condition $(\dagger 1)$ does not hold. In this case we may assume that $\hat{l}(\hat{X}) \geq 2$ by $(\mathrm{B})$. Then we can take $f_{1}: X_{1} \rightarrow \hat{X}$ as in (9.13)(2). Since $U$ in $(9.13)(2)$ satisfies $l(U)=l-1$, there is a $K_{X_{1}}+\mathcal{H}_{X_{1}}$ crepant Gorenstein resolution $g_{1}: \hat{Y} \rightarrow X_{1}$ as in (9.19) or (9.8). Let 
$f: \hat{Y} \rightarrow \hat{X}$ be the composition. We see that $\# \mathcal{E}(f)=\# \mathcal{E}\left(f_{1}\right)+\# \mathcal{E}\left(g_{1}\right) \leq$ $\left(\hat{w}_{1}(\hat{X})+2\right)+2 \operatorname{aw}(U)=2 \operatorname{aw}(\hat{X})+1$. We also see that

$$
\begin{aligned}
\# \mathcal{E}\left(f, \hat{\Delta}_{\hat{X}}(k),(k+1) / 4\right)= & \# \mathcal{E}\left(f_{1}, \hat{\Delta}_{\hat{X}}(k),(k+1) / 4\right)=1, \\
\# \mathcal{E}\left(f, \Delta_{X}(k),(k+3) / 4\right)= & \# \mathcal{E}\left(g_{1}, \Delta_{U}(k),(k+2) / 4\right) \\
& = \begin{cases}1 & \text { if }(\dagger 3) \text { does not hold, } \\
2 & \text { if }(\dagger 3) \text { holds, }\end{cases} \\
\# \mathcal{E}\left(f, \hat{\Delta}_{\hat{X}}(k),(k+1) / 2\right)= & \# \mathcal{E}\left(f_{1}, \hat{\Delta}_{\hat{X}}(k),(k+1) / 2\right) \\
= & \left(\hat{w}_{1}(\hat{X})+1\right) / 2, \\
\# \mathcal{E}\left(f, \hat{\Delta}_{\hat{X}}(k),(k+2) / 2\right)= & \# \mathcal{E}\left(f_{1}, \hat{\Delta}_{\hat{X}}(k),(k+2) / 2\right) \\
& \quad+\# \mathcal{E}\left(g_{1}, \Delta_{U}(k),(k+1) / 2\right) \\
= & \left(\hat{w}_{1}(\hat{X})+1\right) / 2+\left(\bar{w}_{1}(U)-1\right) / 2 \\
& =\left\lfloor\hat{w}_{2}(\hat{X}) / 2\right\rfloor,
\end{aligned}
$$

and other $E \in \mathcal{E}(f)$ satisfies $a\left(E, X, \Delta_{X}(k)\right) \geq(k+5) / 4$ or $\geq(k+3) / 2$. Hence $f: Y \rightarrow X$ satisfies the conditions in (9.20).

We can prove similarly in the case where the condition $(\dagger 1)$ holds.

Corollary 9.21. Let $X, w_{i}(X), \mathcal{H}_{X}$ be as in (9.1) and (9.3). Then there is a $K_{X}+\mathcal{H}_{X}$-crepant Gorenstein resolution $f: Y \rightarrow X$ such that $\# \mathcal{E}(f) \leq 2 \operatorname{aw}(X)$ with

$$
\begin{aligned}
\# \mathcal{E}(f, 1 / 2) & = \begin{cases}\left(\bar{w}_{1}(X)+1\right) / 2 & \text { if }(* 2) \text { does not hold }, \\
\left(\bar{w}_{2}(X)+3\right) / 2 & \text { if }(* 2) \text { holds, }\end{cases} \\
\# \mathcal{E}(f, 1) & = \begin{cases}w_{2}(X) / 2 & \text { if }(* 4) \text { does not hold } \\
w_{2}(X) / 2+1 & \text { if }(* 4) \text { holds },\end{cases}
\end{aligned}
$$

and other $E \in \mathcal{E}(f)$ satisfies $a(E, X)>1$.

Proof. This comes from (9.19) by setting $k=0$ if $X$ satisfies $w_{3}(X) \geq 3$ or $\bar{w}_{1}(X)=1, w_{2}(X) \geq 4$. Otherwise we can apply (9.7) or (9.8) to obtain this result.

Corollary 9.22. Let $\hat{X}, \hat{w}_{i}(\hat{X}), \mathcal{H}_{\hat{X}}$ be as in (9.4) and (9.6). Then there is a $K_{\hat{X}}+\mathcal{H}_{\hat{X}}$-crepant Gorenstein resolution $f: \hat{Y} \rightarrow \hat{X}$ such that 


$$
\begin{aligned}
& \# \mathcal{E}(f) \leq 2 \operatorname{aw}(\hat{X})+1 \text { with } \\
& \# \mathcal{E}(f, 1 / 4)= \begin{cases}1 & \text { if }(\dagger 1) \text { does not hold, } \\
2 & \text { if }(\dagger 1) \text { holds, }\end{cases} \\
& \# \mathcal{E}(f, 3 / 4)= \begin{cases}1 & \text { if }(\dagger 3) \text { does not hold, } \\
2 & \text { if }(\dagger 3) \text { holds, }\end{cases} \\
& \# \mathcal{E}(f, 1 / 2)=\left(\hat{w}_{1}(\hat{X})+1\right) / 2, \quad \# \mathcal{E}(f, 1)=\left\lfloor\hat{w}_{2}(\hat{X}) / 2\right\rfloor,
\end{aligned}
$$

and other $E \in \mathcal{E}(f)$ satisfies $a(E, \hat{X})>1$.

Proof. This follows from (9.20) by setting $k=0$.

Remark 9.23. (9.21) and (9.22) prove our main results (3.1), (3.2) and (3.3) for $X$ as in (9.1) and $\hat{X}$ as in (9.4). In this case $f_{\mid D_{Y}}: D_{Y}=f_{*}^{-1} D_{X} \rightarrow$ $D_{X}$ need not be the minimal resolution of $D_{X}$ even if $D_{X} \in \mathcal{H}_{X}$ has only a rational double point. We also see that exceptional prime divisors may not be irreducible when it is restricted to $D_{Y}$.

\section{REFERENCES}

[Alex94] V. Alexeev, General elephants of $\mathbb{Q}$-Fano 3-folds, Compositio Math., 91 (1994), 91-116.

[Cor95] A. Corti, Factoring birational maps of threefolds after Sarkisov, J. Algebraic Geometry, 4 (1995), 223-254.

[Cor00] Singularities of linear systems and 3-fold birational geometry, Explicit birational geometry of 3-folds, Cambridge LMS., 281 (2000), pp. 259-312.

[Hay99] T. Hayakawa, Blowing ups of 3-dimensional terminal singularities, Publ. RIMS, Kyoto Univ., 35 (1999), 515-570.

[Hay00] — Blowing ups of 3-dimensional terminal singularities, II, Publ. RIMS, Kyoto Univ., 36 (2000), 423-456.

[Hay05] — A remark on partial resolutions of 3-dimensional terminal singularities, Nagoya Math. J., 178 (2005), 117-127.

[Kaw93] Y. Kawamata, The minimal discrepancy of a 3-fold terminal singularity, Appendix to [Sho93].

[Kaw96] — Divisorial contractions to 3-dimensional terminal quotient singularities, Higher-dimensional complex varieties (Trento, 1994), de Gruyter (1996), pp. 241-246.

[Mori85] S. Mori, On 3-dimensional terminal singularities, Nagoya Math. J., 98 (1985), 43-66.

[Reid87] M. Reid, Young person's guide to canonical singularities, Algebraic Geometry, Bowdoin 1985, Proc. Symp. Pure Math., 46 (1987), pp. 345-416. 
[Sho85] V. Shokurov, The nonvanishing theorem, Math. USSR Izv., 19 (1985), 591-604.

[Sho93] — 3-fold log flips, Russian Acad. Sci. Izv. Math., 40 (1993), 95-202.

[Sho96] — 3-fold log models, J. Math. Sci., 81 (1996), 2667-2699.

Department of Mathematics

Kanazawa University

Kakuma-machi

Kanazawa, 920-1192

Japan

thaya@kenroku.kanazawa-u.ac.jp 